THE INTERACTIONS BETWEEN COGNITIVE CONTROL, AGING, AND EMOTION by

Linda Truong

M.A. in Psychology, Ryerson University, 2011

B.Sc. (Hons), Specialist in Psychology, University of Toronto, 2008

\author{
A dissertation \\ presented to Ryerson University \\ in partial fulfillment of the \\ requirements for the degree of \\ Doctor of Philosophy \\ in the Program of \\ Psychology
}

Toronto, Ontario, Canada, 2015

(C) Linda Truong 2015 


\section{AUTHOR'S DECLARATION FOR ELECTRONIC SUBMISSION OF A DISSERTATION}

I hereby declare that I am the sole author of this dissertation. This is a true copy of the dissertation, including any required final revisions, as accepted by my examiners.

I authorize Ryerson University to lend this dissertation to other institutions or individuals for the purpose of scholarly research

I further authorize Ryerson University to reproduce this dissertation by photocopying or by other means, in total or in part, at the request of other institutions or individuals for the purpose of scholarly research.

I understand that my dissertation may be made electronically available to the public. 
The Interactions between Cognitive Control, Aging, and Emotion

Doctor of Philosophy, 2015

\title{
Linda Truong
}

Psychology, Ryerson University

\begin{abstract}
The dual mechanisms of control framework proposes that age-related declines in cognitive control are due to deficits with continuous goal maintenance (proactive control). Older adults default instead to another form of control (reactive control). In contrast to these declines, older adults demonstrate preserved emotional processing. According to the socioemotional selectivity theory, perceived time constraints related to advancing age results in emotional regulation goals in which older adults prioritize positive well-being or mood. To achieve this, they devote more cognitive resources and pay greater attention to positive versus negative information ("positivity effects”) than younger adults. Research on the interactions between cognitive control and emotion is increasing but work focused on the interactions in older adults is limited. Thus, it is unknown how older adults' emotional goals may influence their goal maintenance deficits. This study manipulated mood and emotional face stimuli to examine whether these factors affect age differences in cognitive control between younger (ages 18-30) and older adults (ages 65+). Experiment 1 induced neutral or negative moods prior to a cognitive control task (the standard letter AX-CPT task). Results indicated typical patterns of proactive control in younger adults and reactive control in older adults that did not vary substantially by mood. Experiment 2 examined the effects of neutral, negative, and positive mood inductions on a less cognitively demanding version of the AX-CPT (with face cues as contextual information). Results showed evidence of enhanced proactive control in older adults that was comparable to that of younger adults across
\end{abstract}


all mood conditions, although this was limited to response time data. Additionally, there was evidence of small mood effects on cognitive control. Finally, Experiment 3 examined the effect of positive, negative, and neutral contextual information (face cues) on older adults' cognitive control performance using a different variant of the AX-CPT (face AX-CPT). Results indicated strong engagement in reactive control that did not vary by the emotionality of the contextual information. Together, the results of this study suggest that older adults’ proactive control patterns are affected by the task demands of the AX-CPT, but there is less evidence of mood or emotional stimuli effects. 


\section{Acknowledgements}

This dissertation and my graduate school career would not be possible without my supervisor, Dr. Lixia Yang. She has provided me with tremendous support throughout the years. I would like to thank her for always encouraging me to pursue my ideas. I am grateful to have shared her enthusiasm for the study of aging and I strive to continue this work. I would also like

to thank Dr. Julia Spaniol for being on my supervisory committee and for her thought-provoking comments on theory and cognitive aging throughout my graduate training.

I would also like to thank my lab mates and peers for their companionship and enthusiastic support of my ideas and helping to successfully launch Get Psyched and promote knowledge exchange with older adults. I am also very grateful for all the research assistants involved in data collection for this dissertation throughout the years. I am especially thankful to the many research volunteers who participated in this study.

Finally, I must thank my family (Mom, Dad, Michael, Cathy) for their continual support of my educational goals. A very special thank you to my lifelong partner, Michael, for being the most patient and committed partner throughout my many, many years of school and for introducing Emu into our lives. May we all grow older, together! 


\section{Dedication}

To all of us fortunate enough to grow older - remember, only the survivors grow old. 
Chapter 1: Introduction .................................................................................................................... 1

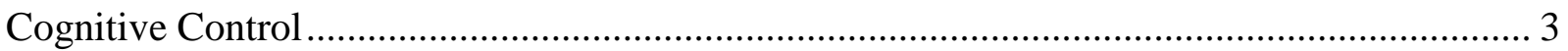

Factors Affecting Cognitive Control.................................................................................... 11

Aging, Emotion, and Cognitive Control ............................................................................... 22

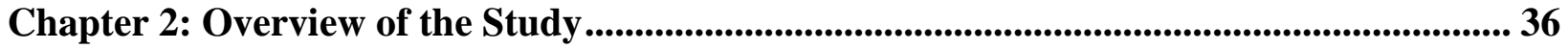

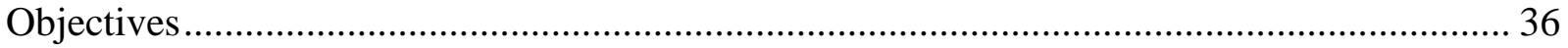

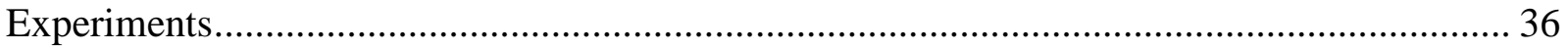

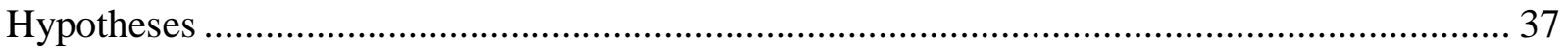

Chapter 3: General Methods ............................................................................................................ 39

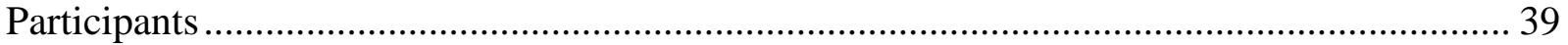

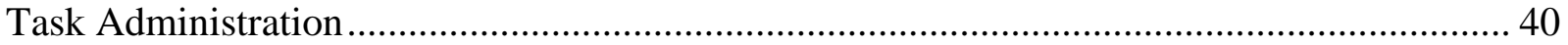

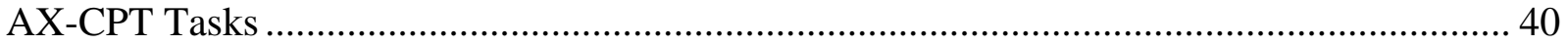

Mood Induction and Ratings .............................................................................................. 41

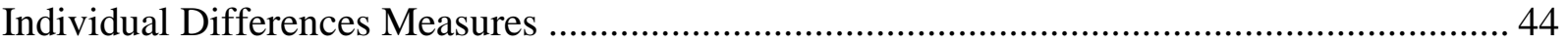

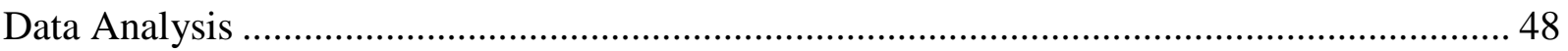

Chapter 4: Experiments 1A and 1B Age Differences in Letter AX-CPT Task under Neutral and Negative Mood .................................................................................................................. 52

Experiment 1A: Age Differences in Letter AX-CPT Task under Neutral Mood ...................... 52

Experiment 1B: Age Differences in Letter AX-CPT Task under Negative Mood ..................... 53

Experiment 1A: Age Differences in Letter AX-CPT Task under Neutral Mood ................... 55

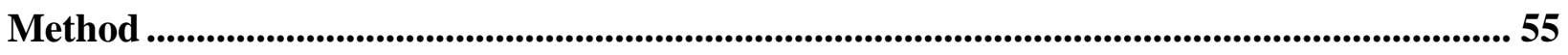

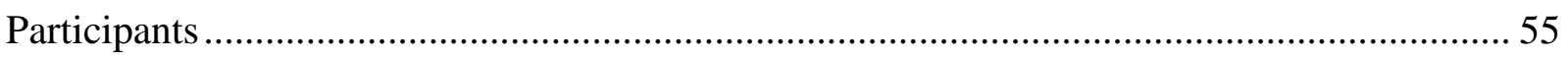

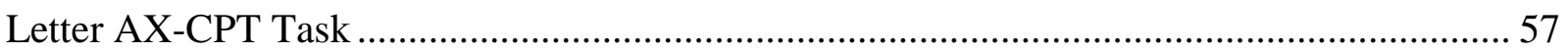

Mood Ratings and Induction............................................................................................. 58

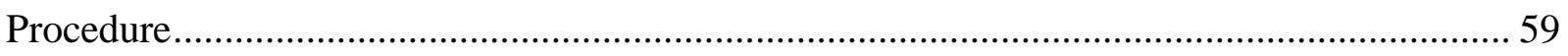

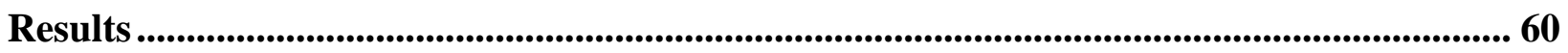

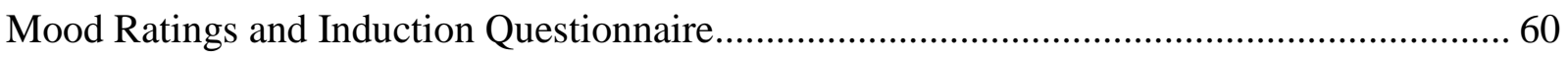

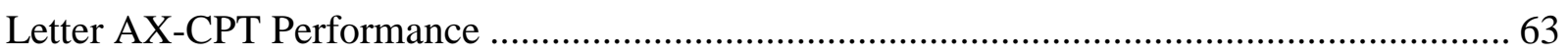

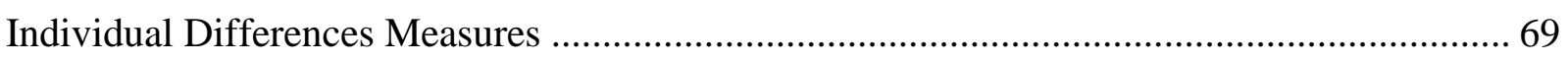




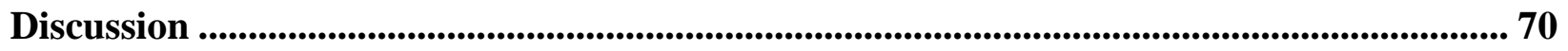

Experiment 1B: Age Differences in Letter AX-CPT Task under Negative Mood.................. 73

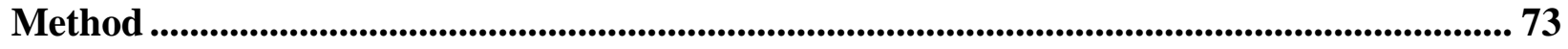

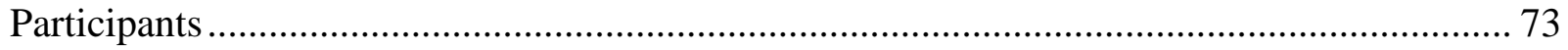

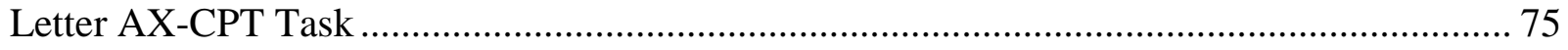

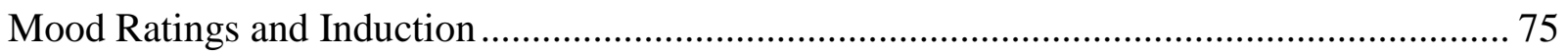

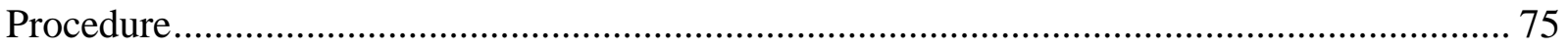

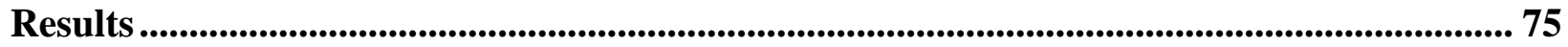

Mood Ratings and Induction Questionnaire........................................................................... 75

AX-CPT Performance ......................................................................................................... 77

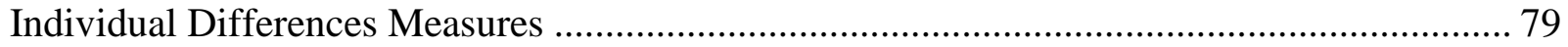

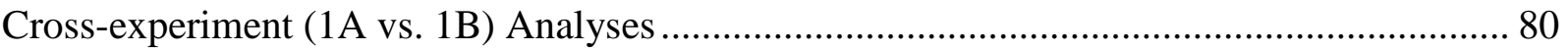

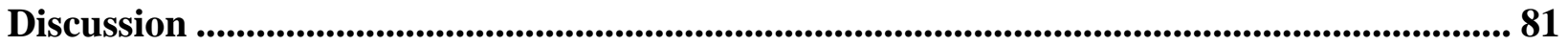

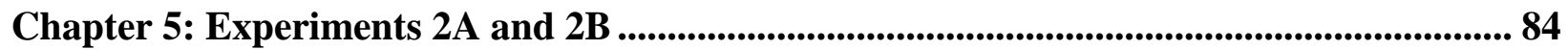

Age Differences in Face-Letter AX-CPT under Neutral and Emotional Mood .................... 84

Experiment 2A: Age Differences in Face-Letter AX-CPT under Neutral Mood ....................... 84

Experiment 2B: Age Differences in Face-Letter AX-CPT under Negative and Positive Mood

Experiment 2A: Age Differences in Face-Letter AX-CPT under Neutral Mood ................... 89

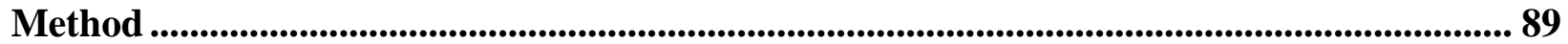

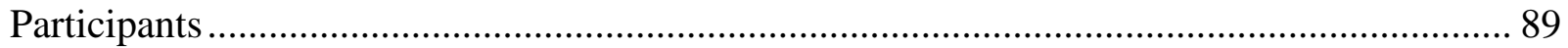

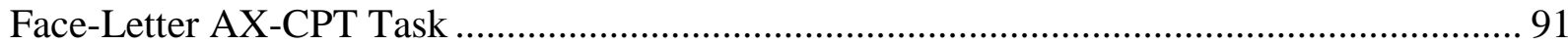

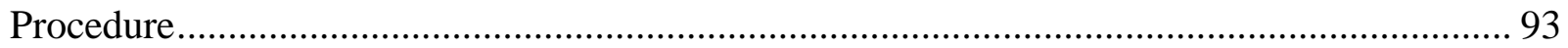

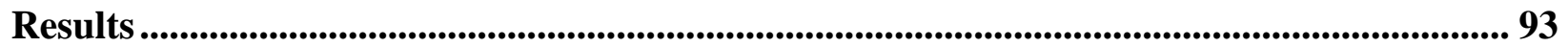

Mood Ratings and Induction Questionnaire....................................................................... 93

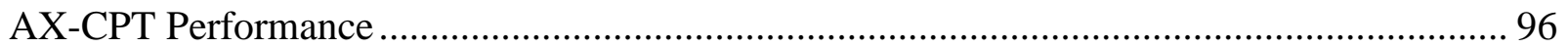

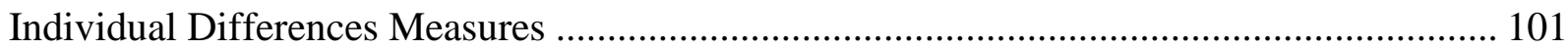

Cross-Experiment (1A vs. 2A) Analyses ........................................................................... 102

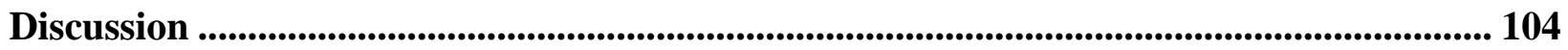

Experiment 2B: Age Differences in Face-Letter AX-CPT under Negative and Positive Mood............................................................................................................................................ 110

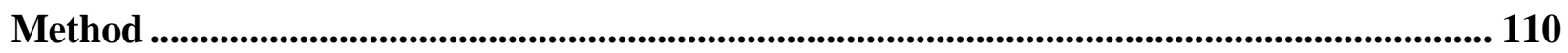

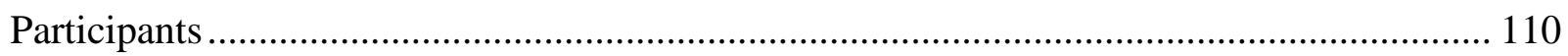

viii 


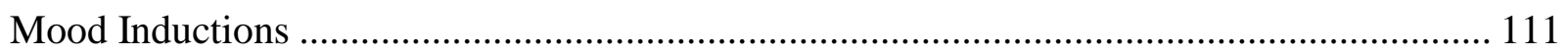

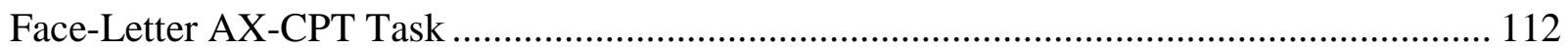

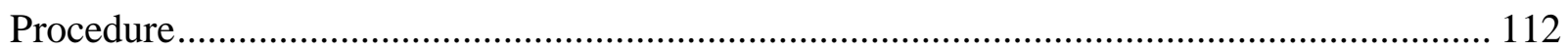

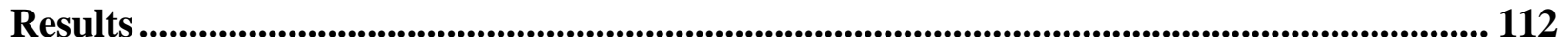

Mood Ratings and Induction Questionnaire........................................................................ 112

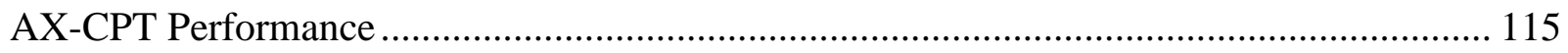

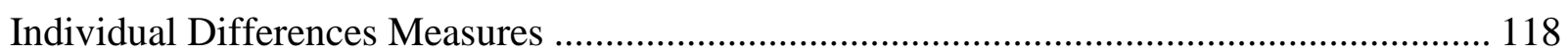

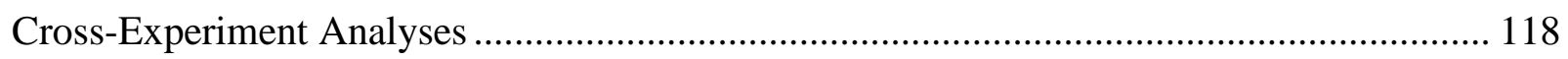

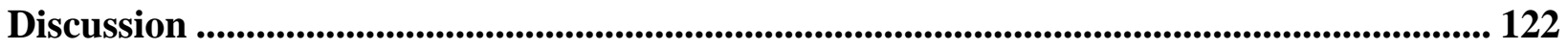

Chapter 6: Experiment 3: Effect of Neutral and Emotional Faces in Face AX-CPT Task 126

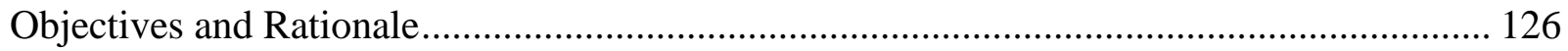

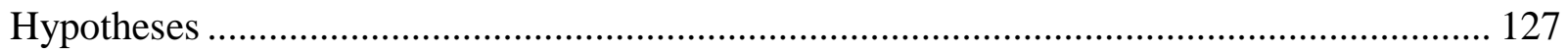

Method ....................................................................................................................................... 127

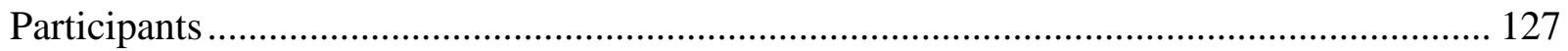

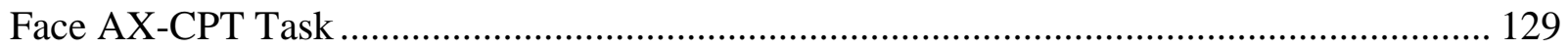

Additional Measures/Questionnaires ………………………........................................... 132

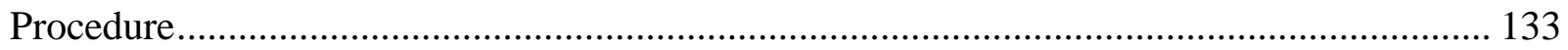

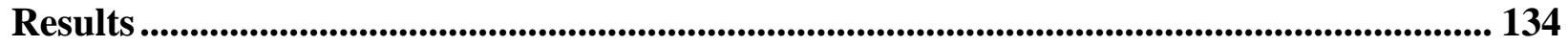

Face AX-CPT Performance …………………………................................................ 134

Individual Differences Measures ……………………….............................................. 138

Discussion ........................................................................................................................................... 140

Chapter 7: General Discussion ...................................................................................................... 142

Age Differences in Cognitive Control ............................................................................ 142

Emotion and Cognitive Control ...................................................................................... 144

Aging, Emotion, and Cognitive Control ......................................................................... 145

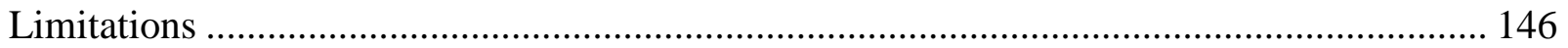

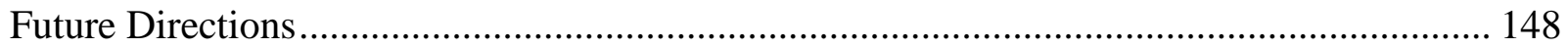

Appendices............................................................................................................................... 150

References.......................................................................................................................................... 171 


\section{List of Tables}

\section{Chapter 3}

Table 1: Characteristics of Mood Induction Pictures ........................................................ 42

\section{Chapter 4}

Table 2: Participant Demographic Characteristics and Cognitive Performance (Exp. 1A) ......... 56

Table 3: Participant Demographic Characteristics and Cognitive Performance (Exp. 1B)......... 74

\section{Chapter 5}

Table 4: Participant Demographic Characteristics and Cognitive Performance (Exp. 2A) ......... 90

Table 5: Rater Accuracies for the Emotional Expression of Selected Faces ............................. 92

Table 6: Participant Demographic Characteristics and Cognitive Performance (Exp. 2B)........ 111

\section{Chapter 6}

Table 7: Participant Demographic Characteristics and Cognitive Performance (Exp. 3) .......... 128 


\section{List of Figures}

\section{Chapter 1}



\section{Chapter 3}

Figure 2. Self-Assessment Manikins (SAM) ............................................................... 43

\section{Chapter 4}

Figure 3. Sample illustration of a target trial .............................................................. 57

Figure 4. Mean valence ratings of mood across time point (Exp. 1A \& 1B) ........................... 61

Figure 5. Mean arousal ratings of mood across time point (Exp. 1A \& 1B) ............................ 62

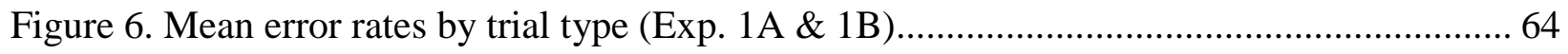

Figure 7. Mean z-transformed RTs by trial type (Exp. 1A \& 1B) ........................................... 65

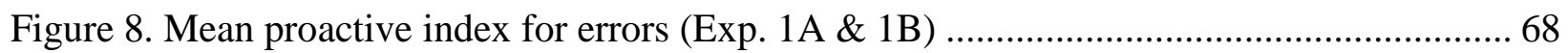

Figure 9. Mean proactive index for median RTs (Exp. 1A \& 1B) ........................................... 69

\section{Chapter 5}

Figure 10. Sample illustration of a target trial in the male block ........................................... 93

Figure 11. Mean valence ratings of mood across time point (Exp. 2A \& 2B) .......................... 94

Figure 12. Mean arousal ratings of mood across time point (Exp. 2A \& 2B) ........................... 95

Figure 13. Mean error rates by trial type (Exp. 2A \& 2B) ................................................... 97

Figure 14. Mean z-transformed RTs by trial type (Exp. 2A \& 2B) ......................................... 98

Figure 15. Mean proactive index for errors (Exp. 2A \& 2B) .............................................. 100

Figure 16. Mean proactive index for RTs (Exp. 2A \& 2B) ................................................ 101

\section{Chapter 6}

Figure 17. Sample illustration of a target trial in the positive block. 
Figure 18. Mean error rates by trial type across the three blocks (Exp. 3) .............................. 135

Figure 19. Mean z-transformed median RTs by trial type across the three blocks (Exp. 3) ...... 136

Figure 20. Mean proactive index for errors and RTs (Exp. 3)........................................... 138 


\section{List of Appendices}

Appendix I: Ryerson REB Approval Letter (REB 2013-286)............................................. 150

Appendix II: Consent Form-Younger Adults (Experiment 1) .............................................. 151

Appendix III: Consent Form-Older Adults (Experiments 1 \& 2)........................................... 154

Appendix IV: Debriefing Form (Experiment 1) ......................................................... 157

Appendix V: Consent Form-Younger Adults (Experiment 2)............................................ 158

Appendix VI: Debriefing Form (Experiment 2) …......................................................... 161

Appendix VII: Consent Form (Experiment 3) ................................................................... 162

Appendix VIII: Debriefing Form (Experiment 3) ........................................................... 165

Appendix IX: Mood Induction Questionnaire ................................................................. 166

Appendix X: Correlations between AX-CPT Performance and Cognitive Tests...................... 168

Appendix XI: AX-CPT Median Response Times ............................................................. 169

Appendix XII: Face AX-CPT Questionnaire................................................................ 170 


\section{Chapter 1: Introduction}

Cognitive control is the ability to guide processing and behavior in order to accomplish task goals, often in the presence of interference from goal-irrelevant information (Diamond, 2013; Jurado \& Rosselli, 2007). This is typically accomplished through successful maintenance, updating, and shifting of task goals (Braver, Gray, \& Burgess, 2007; Kane \& Engle, 2002). Cognitive control has been implicated in a variety of cognitive abilities, including fluid intelligence (Salthouse, Atkinson, \& Berish, 2003) and long-term memory (Adrover-Roig, Sesé, Barceló, \& Palmer, 2012). Thus, it is particularly concerning that research shows significant cognitive control deficits with advancing age (Diamond, 2013). Such research demonstrates that older adults are less successful at tasks requiring a high degree of cognitive control. They are often less accurate, slower, and less efficient overall than their younger counterparts. However, this depiction of cognitive control decline contrasts starkly with an abundance of research on preserved emotional processing and functioning in older age (Carstensen, Pasupathi, Mayr, \& Nesselroade, 2000; Scheibe \& Carstensen, 2010). Older adults demonstrate comparable performance to younger adults when tasks contain emotional relative to neutral materials (Löckenhoff \& Carstensen, 2007) and sometimes demonstrate enhanced performance for positive information (Mather \& Carstensen, 2005).

These two bodies of literature have oftentimes been contrasted as research on "cold" cognitive or executive control/functioning versus "hot" emotional functioning. Examination of “cold” cognition is often conducted using neutral materials, such as letter and number stimuli, to control for potential confounding effects of other variables. In contrast, research on emotional functioning has traditionally focused on the regulation of emotions - such as strategies to deal with negative affect - without taking into consideration cognitive functioning. Historically, these 
differences have resulted in limited research on the interactions of "cold" and "hot" abilities. This is changing as researchers are increasingly examining the relationship between such abilities, in line with efforts to understand cognitive functioning in the context of real-life experiences that are often emotional in nature (Hess, 2005). Such initiatives have been supported with the use of increasingly advanced neuroimaging techniques, which have demonstrated many connections between regions that are thought to play roles in these two domains of functioning (Dolcos, Iordan, \& Dolcos, 2011).

Although researchers are increasingly interested in studying the interactions between cognitive control and emotional functioning, work in this area has primarily been conducted with younger adults. The lack of research on older adults is an important and critical issue to address, considering findings of age-related preservations of emotional processing in the face of cognitive control declines. This dissertation contributes to this area of work by examining the interactions between cognitive control and emotion and how they differ between younger and older adults, across three experiments. Two theories serve as the foundations of this work: (1) the dual mechanisms of control framework (Braver, 2012; Braver et al., 2007), which postulates specific age-related changes to a specific mode of cognitive control (proactive control); and (2) the socioemotional selectivity theory (Carstensen, Isaacowitz, \& Charles, 1999), which posits agerelated motivational changes that affect emotional processing and regulation. The following literature review will begin by outlining each theory and the accompanying research that has been conducted to test theoretical predictions. This will be followed by a review of the evidence suggesting important interactions between cognitive control, emotion, and aging that ultimately form and guide the rationale for this dissertation. 


\section{Cognitive Control}

As stated, aging is hypothesized to be associated with changes to the efficiency with which goals are processed. Research has found that older adults have difficulties with maintaining, shifting, and updating task goals in working memory (Braver et al., 2001; Braver, Satpute, Rush, Racine, \& Barch, 2005). This dissertation focuses on one cognitive control theory - the dual mechanisms of control (DMC) framework (Braver, 2012; Braver et al., 2007) - that outlines how processing of contextual information (i.e., any task-relevant information that can bias processing and behaviour, such as goal representations) changes with age. Specifically, the DMC account posits that advancing age leads to a strategy shift in controlled processing, from one that relies on proactive maintenance of context (proactive control) to one that relies on reactivation (reactive control) instead (Braver et al., 2001; Braver \& West, 2008). The following section describes the DMC framework and details the moderator factors of age and emotion on cognitive control that are most pertinent to this dissertation.

Dual mechanisms of control framework. Successful cognitive control is believed to depend on attention and processing, internal representation, maintenance (in working memory), and updating of context information (Braver \& Barch, 2002; Braver \& West, 2008). Context is defined as task or goal-relevant representations that can bias or guide processing. As such, cognitive control allows us to exert inhibitory control over task-irrelevant information, which is particularly important when there is a high degree of interference from irrelevant information or conflict between competing responses. The dual mechanisms of control (DMC) framework (Braver, 2012; Braver et al., 2007) postulates that there are two control modes in which one can successfully exert cognitive control in the presence of interference. The first mode - proactive control - relies on continuous maintenance of context to minimize the impact of interference, 
prior to its occurrence. In contrast, the second mode - termed reactive control - resolves interference at the time of its occurrence through a reactivation of context. The DMC framework proposes that most cognitive control tasks are likely completed through a combination of both strategies, although some factors, such as age, may increase the likelihood that individuals will engage more in one strategy over the other. Indeed, proponents of this account have asserted that it is unlikely that the two modes are mutually exclusive (Braver, 2012) and that the most cognitively demanding tasks likely require the use of both modes for efficient performance (Braver, Paxton, Locke, \& Barch, 2009). Furthermore, the DMC model proposes that proactive and reactive control may potentially be independent mechanisms which can both be engaged at the same time (Braver, 2012; Braver et al., 2007). Although research examining these two modes is growing, in-depth examinations are still in relatively early stages. As such, the proposed independence of the modes has not been directly confirmed. More conclusive evidence, such as findings of a double dissociation of proactive and reactive control (i.e., modulate one without affecting the other), has not been identified. Such evidence will be necessary to further refine conceptualizations of the constructs (Braver, 2012).

Although the independence of proactive and reactive control modes has not been confirmed, the DMC hypothesizes that each have unique properties that distinguish the two modes. To best demonstrate these differences, the following section will begin by describing the AX-version of the Continuous Performance Test (CPT; Rosvold, Mirsky, Sarason, Bransome, \& Beck, 1956), hereafter referred to as the AX-CPT task (Cohen, Barch, Carter, \& ServanSchreiber, 1999). This is a cognitive control paradigm that is typically used for its ability to provide behavioural markers of proactive and reactive control. The AX-CPT task is a main focus of this dissertation as it has been the standard task used to examine the two control modes (e.g., 
Braver et al., 2001, 2005; Haarmann, Ashling, Davelaar, \& Usher, 2005; Paxton, Barch, Racine, \& Braver, 2008; Paxton, Barch, Storandt, \& Braver, 2006; Rush, Barch, \& Braver, 2006) ${ }^{1}$. Describing this task will help demonstrate the differences in the two modes and how they are often measured. The properties that distinguish proactive from reactive control will be further detailed. This will be followed by a focused review of research that has revealed how certain factors -age and emotion, in particular - influence relative engagement in proactive and reactive control.

\footnotetext{
${ }^{1}$ Although the AX-CPT task has been the standard task used by proponents of the DMC thus far, researchers have also used other traditional paradigms of cognitive control/executive functioning, such as the Stroop task (e.g., Bugg, 2014), go-no-go task (e.g., Staub, Doignon-Camus, Bacon, \& Bonnefond, 2014); task-switching paradigms (e.g., Jimura \& Braver, 2010), and probe-recency Sternberg tasks (e.g., Manard, Carabin, Jaspar, \& Collette, 2014), to examine proactive and reactive control in younger and older adults.
} 
$\boldsymbol{A X}$-CPT task. The AX-CPT task uses task cues and ambiguous probes to measure goal representation, maintenance, and updating (Figure 1).

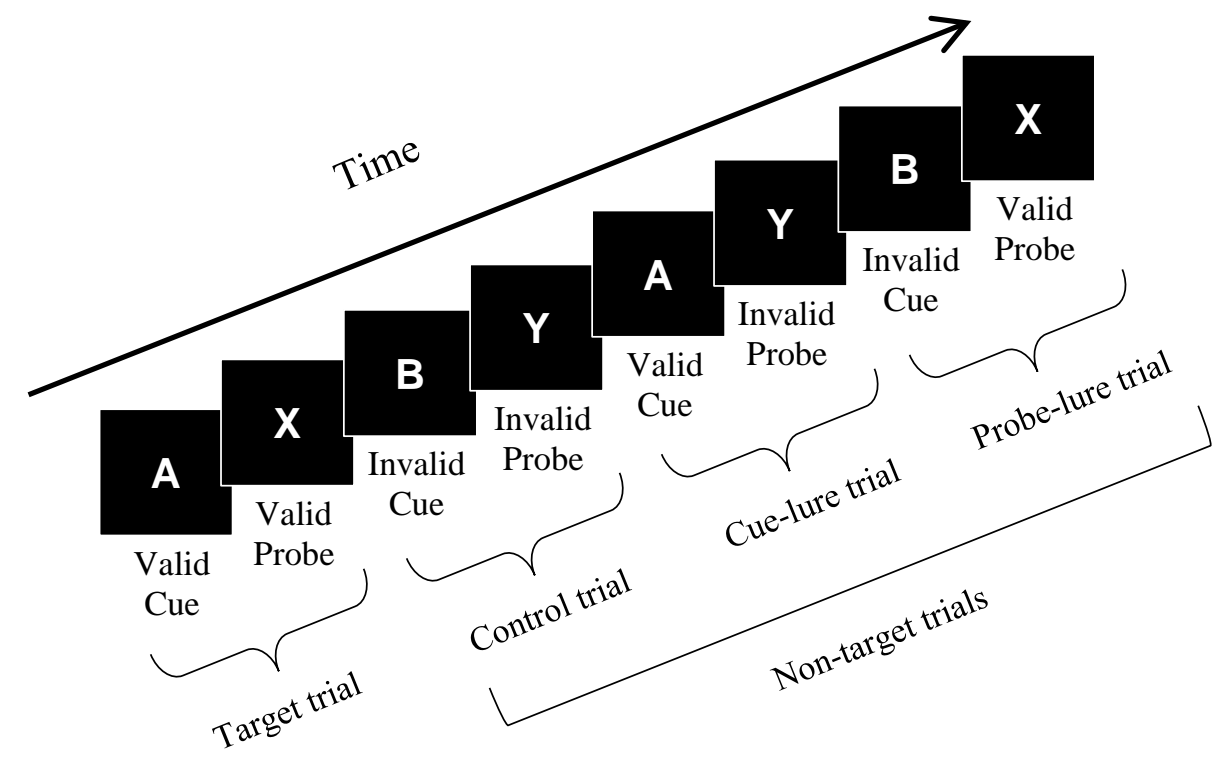

Figure 1. Sample illustration of AX-CPT trials. Letters are presented one at a time and each trial consists of a cue-probe pairing. Frequencies of trial types: target (70\%), control (10\%), cue-lure (10\%), and probe-lure (10\%). Letters B and Y represent any letters of the alphabet that are not the letters $\mathrm{A}$ or $\mathrm{X}$.

In this task, letter stimuli are presented continuously, one at a time. These letters belong to one of four categories: valid cues, invalid cues, valid probes, and invalid probes. Cues are presented first, followed by a brief delay prior to the presentation of a probe. Target responses are required to a valid probe letter (“X”) only when it follows a valid cue letter (“A”, i.e., AX target trials); non-target responses are required to all types of cues (valid or invalid) and the probes in all other combinations (i.e., invalid cue and valid probe BX, valid cue and invalid probe AY, invalid cue and invalid probe BY trials). These AX target trials occur frequently, comprising $70 \%$ of all trials. This frequency distribution creates a strong response bias towards 
target trials and renders the cue " $\mathrm{A}$ " to be a strong predictor of a subsequent " $\mathrm{X}$ ” probe. The remaining $30 \%$ of trials are evenly distributed across three types of non-target trials: AY, BX, and BY trials. On AY trials, participants see a valid cue “A”, followed by an invalid probe (Y). The opposite combination occurs for BX trials: an invalid cue (B) is followed by a valid "X" probe. AY and BX non-target trials generate interference: in AY trials, the valid cue "A” may generate anticipation of a target response, thus causing a greater likelihood of false-alarms or slower responses to the invalid probe; these are referred to as cue-lure trials. In contrast, interference from the valid " $\mathrm{X}$ ” probes may also result in poor performance on BX trials if one did not maintain the cue, causing them to forget, or be uncertain, that the " $\mathrm{X}$ " was preceded by an invalid cue; these are referred to as probe-lure trials. The valid " $\mathrm{X}$ " may cause a greater likelihood of false alarms or accurate but slow rejection responses. Finally, BY trials are treated as non-target control trials that do not generate interference (or only low levels of interference), as both cues and probes are invalid and it is clear that a non-target response is the appropriate action. Performance on interference trials can be compared to these control trials to assess the degree of interference arising from them.

Performance on the AX-CPT task can be used to infer the degree of engagement in proactive versus reactive control (Braver et al., 2007). The critical markers are performance differences between cue-lure and probe-lure interference trials, relative to the other trial types. Specifically, poorer performance (i.e., higher error rates and slower response times) on cue-lure trials compared to the other trial types - particularly probe-lure trials - are hypothesized to be markers of proactive control. In contrast, poorer performance on probe- relative to cue-lure trials indexes the use of reactive control. The following sections on proactive and reactive control will further describe how these performance differences on the AX-CPT task distinguish between the 
two control modes. It will also describe some of the properties that define each mode and some of the supporting evidence that has been found thus far.

Proactive control. The DMC framework defines proactive control as a mode in which context (e.g., task goals) are actively and continuously maintained in working memory, typically over a brief period of time (Braver et al., 2007). This active maintenance of contextual information such as task goals allows one to effectively focus one's attention and control on information that is relevant to the task, while ignoring task-irrelevant information. This top-down anticipatory strategy of proactive control can help prevent or minimize the effects of interference from goal-irrelevant information, prior to its occurrence. In the AX-CPT task, cue letters convey contextual goal-relevant information. For example, a valid cue " $\mathrm{A}$ " is a signal to prepare a target response, in anticipation of a valid probe "X" that frequently follows such cues (i.e., AX target trials). In contrast, invalid cue letters on BX probe-lure and BY control trials signal that one should prepare a non-target response, as target responses are only required when valid cues are presented. Such continuous preparation beginning at the onset of the cue should result in better performance (higher accuracy and faster responses) on target, probe-lure, and control trials. Neuroimaging has also provided evidence that such anticipatory proactive processing is reflected in the brain as sustained activation of the lateral prefrontal cortex during a delay period, occurring after presentation of a cue (Braver et al., 2009).

Although engaging in proactive control has the advantages of higher accuracy and/or faster responses on most trials, this control mode increases the likelihood for poorer performance (lower accuracy and slower response times) on cue-lure AY trials, which require a non-target response (Braver et al., 2001, 2005). If one is effectively using proactive control, the valid cue "A" should signal that a target response should be prepared and the task goal ("TARGET 
response only if ' $\mathrm{X}$ ' follows') should be actively maintained over the delay period. As target trials occur at a high frequency, a strong response bias to respond "target" upon detection of a valid cue is generated because the valid cue is highly predictive of an upcoming valid probe. When an invalid probe appears instead, the prepared target response must be inhibited and replaced with a non-target response. Overcoming this strong response bias may result in one of two outcomes: (a) longer response times to reject the invalid probe; or (b) if not successfully overcome, lower accuracy rates due to false-alarms to the invalid probe. Thus, poor performance on the cue-lure AY trials becomes a behavioural marker of proactive control, demonstrating evidence of anticipatory responses elicited by the presence of a valid cue. In other words, the valid cue "lures" them to expect a subsequent valid probe. Despite poor cue-lure performance, the DMC argues that engaging in proactive control is still the more effective mode versus reactive control in the AX-CPT task. This is because proactive control will aid the preparation of accurate responses for the majority of trials (i.e., $90 \%$ of all trials).

Finally, another key property of proactive control is that the sustained, continuous maintenance of task goals is resource-demanding. As control is maintained for longer periods of time, engaging in this mode can be costly in terms of cognitive resources, particularly when compared to reactive control. Specifically, Braver et al. (2007) have postulated that engaging in proactive control is likely more metabolically costly than engaging in reactive control. For example, proactive control requires greater attentional resources in order to hold a goal or context representation in working memory for a longer period of time than is likely needed for reactive control. This advance preparation in proactive control is hypothesized to be more cognitively costly. 
Reactive control. In contrast to the sustained activity that characterizes proactive control, the reactive control mode is initiated only after the detection of a cognitively demanding event, such as the occurrence of interference (Braver, 2012; Braver et al., 2007). Rather than maintaining task goals and preparing responses in advance, individuals reactivate task goals as needed, in order to appropriately respond. Thus, it is a later-acting form of correction or interference resolution that relies on bottom-up processing. In the AX-CPT task, individuals engaging in reactive control are presumably not taking advantage of task cues that convey goalrelevant information. Instead, task goals are only reactivated upon occurrence of the probe letter. For example, if the probe letter was valid probe " $X$ ", individuals would reactivate task goals ("TARGET response if preceded by a valid cue") through evaluation of the validity of the cue (valid in AX target trials, invalid in BX probe-lure trials). Performance on AX target trials while engaging in reactive control is typically similar to that while engaging in proactive control; in both cases, a target response is expected to the probe " $\mathrm{X}$ " following the cue " $\mathrm{A}$ ". The difference occurs with interference-generating probe-lure BX trials, with poorer accuracy and slower responses if reactive relative to proactive control is engaged; this is presumably due to the interference resolution processes that must occur in order to respond appropriately. Thus, poorer performance on BX probe-lure trials relative to the other trials is a behavioural marker of the reactive control mode. However, unlike proactive control, performance on cue-lure AY trials is intact - individuals engaging in reactive control will primarily respond based on the invalid probe (i.e., make a NON-TARGET response), thus decreasing the likelihood of false alarms. This control mode is often reflected in the lateral prefrontal cortex as transient (rather than tonic) activity that is probe-based, beginning at the onset of the probe stimulus, rather than at the cue (Braver et al., 2009). 
As task goals are only reactivated in response to probe stimuli (rather than maintained throughout the duration of a trial), the DMC framework predicts that reactive control is less resource-demanding overall than proactive control. By exerting cognitive control only when needed (e.g., in the presence of goal-irrelevant or incongruent interference), use of a reactive strategy is less taxing than the continuous maintenance of proactive control and is hypothesized to be the preferred control mode when cognitive resources are limited.

\section{Factors Affecting Cognitive Control}

One advantage of the DMC framework is the availability of both behavioural and neural markers of proactive and reactive control. This allows researchers to elucidate or manipulate various factors that are hypothesized to influence each control mode. This section outlines the effects of age and emotion, as they are most pertinent to this dissertation. The DMC model predicts that these factors have specific effects on proactive and reactive control (Braver, 2012; Braver et al., 2001, 2007) and a growing body of empirical work has both supported and challenged these tenets.

Aging and cognitive control. The DMC predicts that younger adults will exhibit primarily proactive control (e.g., lower accuracy and/or slower response times on cue-lure versus the other trial types) that will not be observed in older adults (Braver et al., 2001). The DMC model hypothesizes that prefrontal cortex functioning and its interactions with the dopamine neurotransmitter system play significant roles in the exertion of proactive control (Braver et al., 2007). Specifically, dopamine projections to the prefrontal cortex are thought to serve a "gating" function, which regulates access to contextual information within working memory (Braver \& 
Barch, 2002) ${ }^{2}$. As aging is associated with declines in both prefrontal (see Hedden \& Gabrieli, 2004 for a review) and dopaminergic functioning (e.g., Volkow et al., 1998), the DMC predicts that proactive control will be particularly affected by advancing age. Specifically, it predicts that these changes will cause a shift away from the default proactive mode present in younger adults to one that is reactive (Braver et al., 2001, 2005). Indeed, age group comparisons typically reveal that younger adults are more proactive and less reactive than older adults (e.g., Braver et al., 2001, 2005; Paxton, Barch, Storandt, \& Braver, 2006; Rush, Barch, \& Braver, 2006). These patterns are in line with DMC's prediction that those with compromised prefrontal and dopaminergic functioning and lower cognitive resources - such as older adults - will select the less taxing of the two control modes (i.e., reactive control) by default, possibly as a compensatory strategy (Paxton et al., 2008).

To examine these age differences, researchers have frequently used the AX-CPT task. Most studies compare younger and older adults' performance on cue-lure versus probe-lure trials. These studies have found fairly consistent patterns of differences between the two groups in the expected direction, indexed as better cue-lure but poorer probe-lure performance in younger than older adults. For example, Braver et al. (2001) compared younger and older adults on the AX-CPT under three conditions: baseline, degraded, or interference. The baseline condition was the standard AX-CPT, as described earlier. The degraded condition perceptually manipulated the visual clarity of the letter stimuli by degrading $85 \%$ of the pixels that formed a letter. The interference condition consisted of irrelevant distracter letters presented during the cue-delay probe, which is typically unfilled. Both the degraded and interference conditions were

\footnotetext{
${ }^{2}$ A review of the specific role served by the dopamine system within the DMC model is beyond the scope of this dissertation; however, see Braver and Barch, 2002; Braver et al., 2001, 2007 for further details.
} 
intended to increase task difficulty but in different ways to test hypotheses about the underlying mechanism driving age-related changes. Specifically, the degraded condition was expected to increase perceptual demands but was not expected to significantly affect context representation or maintenance across the delay. In contrast, the interference condition was hypothesized to increase task demands by interfering with both context representation and maintenance. This was expected to further tax older adults' cognitive resources, which was hypothesized to accentuate age differences in cognitive control. Results from this study were consistent with these hypotheses. Older adults exhibited reduced proactive but greater reactive control, relative to younger adults, in the baseline condition. This pattern was even more evident in the interference condition, suggesting that the distracter items further taxed older adults' cognitive resources, leading them to engage in more reactive control instead. This finding of exaggerated age differences under increased task demands provide support that older adults' reduced ability to engage in proactive control may be in part attributed to their limited cognitive resources.

Although the DMC model is agnostic as to whether age differences would differ across measures of performance (i.e., accuracy or response times), studies have revealed some inconsistent patterns between accuracy and response time data (Braver et al., 2005). Specifically, the most consistent evidence of age differences in proactive control are manifested as poorer cuelure accuracy performance in younger than older adults (e.g., Braver et al., 2001, 2005; Paxton et al., 2006; Rush et al., 2006). The DMC suggests that this reflects an age-related reduction in proactive control (Braver et al., 2001, 2005). Younger adults appear to be using the valid cue in cue-lure trials to prepare a target response (which leads them to false alarm when an invalid probe is presented afterwards), whereas older adults do not show evidence of this, showing no decrement in performance on cue-lure trials. However, this age difference is not always observed 
(Haarmann et al., 2005; Paxton et al., 2008). Furthermore, evidence of older adults’ reactive control tendencies most consistently manifest in response time but not error data. Specifically, relative to younger adults, older adults are disproportionately slow on probe-lure trials compared to the other trial types, even after controlling for age-related slowing (e.g., Braver et al., 2001, 2005; Paxton et al., 2008, 2006; Rush et al., 2006). Findings of greater probe-lure errors in older than younger adults have been found as well (e.g., Braver et al., 2001; Paxton et al., 2008), but this difference is not consistently found (e.g., Braver et al., 2005; Paxton et al., 2006), or the difference is not statistically significant (e.g., Braver et al., 2001). Why are patterns of age group differences expressed in discrepant ways for errors and response times? Braver and colleagues (2005) have suggested that this disproportionate slowing on probe-lure trials may specifically reflect older adults' tendency to reactivate task goals after presentation of a valid probe in order to determine the correct response (i.e., reactive control). This reactivation is presumed to slow down responses, as compared to when individuals proactively prepare a response in advance, at the moment an invalid cue is presented. This is presumably how younger adults respond to probe-lure trials, as reflected by generally fast responses on these trials. Both strategies lead to the correct response (i.e., reject the probe), but the reactive strategy should take longer to implement than the proactive one.

It should be noted that age differences are also apparent when comparing the relative differences in performance on cue- versus probe-lure trials within an age group, such as studies with only younger (Locke \& Braver, 2008) or older participants (e.g., Paxton et al., 2006, study 2). Consistent with DMC predictions, these studies typically find that younger adults show evidence of greater proactive control engagement, as marked by poorer performance on cue-lure relative to probe-lure trials (e.g., Paxton, Barch, Racine, \& Braver, 2008). Older adults exhibit 
the opposite pattern: poorer performance on probe-lure relative to cue-lure trials, suggesting use of reactive control (e.g., Paxton, Barch, Storandt, \& Braver, 2006; Rush, Barch, \& Braver, 2006). Unfortunately, these analyses on the relative differences between performance on these trials (separately for younger and older adults) are not consistently reported in studies that have examined both age groups. Instead, these studies report only the age group effect on cue- and probe-lure performance.

Although age differences in proactive and reactive control are not always consistent in behavioural data, more consistent and clear patterns of age differences have been found in neuroimaging studies, specifically observed as temporal differences in prefrontal activation between younger and older adults. Within the dorsolateral prefrontal cortex (DL-PFC), younger adults exhibit activity beginning at the time of cue onset (Braver et al., 2009). As the DL-PFC has been implicated in active maintenance of task goals or task set information (Barbey, Koenigs, \& Grafman, 2013), this is evidence that younger adults are processing and making use of the contextual information provided by the cue. They also actively maintain this information across the delay period (Braver et al., 2009). In contrast, older adults do not show this pattern. Instead, older adults show a delay-related decrease in activity in this same region. Additionally, older adults exhibit increased activation in the DL-PFC at the onset of the probe, rather than at the time of cue onset, which is the pattern observed in younger adults (Braver et al., 2009; Paxton et al., 2008). Thus, this suggests the presence of age differences in the temporal dynamics of when control is exerted that occurs within the same region in both age groups. In younger adults, control appears to be exerted at the time of cue processing, whereas older adults exert greater control for probe processing. This is consistent with predictions of the DMC of greater 
cue-focused proactive control in younger adults and greater probe-focused reactive control in older adults.

Age group differences in proactive and reactive control have also been found with children, suggesting a developmental trajectory of control, with a gradual increase of proactive control into young adulthood, followed by more reactive patterns in later adulthood (Lorsbach \& Reimer, 2008, 2010). This suggests that general cognitive abilities or resources may play a role in these age differences. For example, Manard, Carabin, Jaspar, and Collette (2014) showed that the ability to use proactive control in situations of high interference was mediated by performance on fluid intelligence and processing speed measures in both younger and older adults. It has also been found that younger adults with high working memory capacity engaged in greater proactive control relative to those with low working memory capacity (Redick, 2014; Richmond, Redick, Braver, Richmond, \& Redick, 2015). Normal age-related changes often results in lower working memory capacity (see Bopp \& Verhaeghen, 2005, for a meta-analysis), and this may partially explain shifts to the less-taxing reactive mode in older age.

Encouragingly, research has shown that older adults can shift from reactive to proactive control. One study found that giving older adults extended practice on the AX-CPT task resulted in greater proactive control, relative to baseline performance. Proactive control in older adults can also be facilitated through targeted strategy training on the AX-CPT task (Braver et al., 2009; Paxton et al., 2006). In these studies, older adults were trained to explicitly focus on the cue stimuli in the AX-CPT task (e.g., verbalization of the cue and task rules) and repeated instructions. Results showed that training attention and processing towards task cues enabled older adults to have greater proactive control post-training compared to baseline, as evidenced by both decreased probe-lure errors and (theoretically predicted) increased cue-lure errors (Braver et 
al., 2009; Paxton et al., 2006). This shift was also reflected in brain activation patterns - training shifted lateral prefrontal activation such that greater activity was found during the cue and delay than probe processing periods (Braver et al., 2009). These patterns more closely resembled those typically exhibited by younger adults. The authors suggest that older adults may require additional environmental support (e.g., through the provision of strategic training or task reinstructions) or extended practice in order to effectively engage in proactive control. These processes may serve to reduce the cognitive demand required to use proactive control, thus allowing older adults with limited cognitive resources to sustain high levels of goal maintenance. However, the mechanisms underlying this possible reduction in cognitive demand have not been thoroughly tested and it is not clear which situations may be more likely to foster or support proactive control use in older adults.

Insights into the factors that support proactive control arises from a study that found robust proactive control across two experiments in older adults and children, at levels comparable to that of younger adults (Kray, Schmitt, \& Heintz, 2015). The purpose of this study was to facilitate proactive control through verbal labelling of the cue stimuli, which required participants to verbalize each presentation of the valid cue letter A as "A" (Experiment 1) or the valid cue face as “Tom/Ruth” (Experiment 2). Participants verbalized “No” for all invalid cues. This was hypothesized to bias attention toward cue processing, thus leading to greater proactive control. Performance in this condition was compared to a probe labelling and a no labelling control condition. Although probe labelling led to increased reactive control in all age groups, there was no effect of cue labelling on proactive control. Unexpectedly, all participants completed the tasks in both experiments in a fairly proactive manner in general, as evidenced by proactive patterns in all conditions; cue labelling did not further promote proactive control. The 
unexpected finding of proactive control in children and older adults may have been driven by important modifications to the AX-CPT. Specifically, the researchers modified the AX-CPT such that the number of competing invalid cues and probes was substantially reduced compared to the standard AX-CPT. In the standard AX-CPT, the invalid cue and probe can be almost any letter of the alphabet, typically resulting in approximately 20 competitor letters that could serve as either cues or probes. In Kray et al. (2015), the number of invalid letters or faces was reduced to three items (e.g., invalid cue letters G, S, F) that served as only invalid cues or probes - not both. This reduction in the number of competitor items may have significantly reduced the cognitive demands of the AX-CPT task, thus facilitating proactive control in individuals with limited cognitive resources (i.e., children and older adults).

Together, studies examining proactive and reactive control in different age groups reveal age-related shifts from a proactive mode in younger adults to one that is more reactive in older age. However, there is evidence suggesting that older adults are capable of flexibly and dynamically shifting from their default reactive mode to one that is more proactive, although they may need environmental support (e.g., training), or simpler cognitive control tasks, in order to do so. Relatedly, there is evidence that proactive control can be further promoted in younger adults with changes to the AX-CPT task. Such changes include making the cue more salient and distinct from the probe through use of different stimuli for each (words versus pictures; van Wouwe, Band, \& Ridderinkhof, 2009) or presenting cue and probe stimuli in separate spatial locations within the task (Reimer et al., 2015). Thus, the research conducted thus far suggests that cognitive control is amenable to external factors (e.g., changing task parameters), which raises the potential to examine how these factors can encourage more sustained engagement in proactive control (Braver, 2012). 
Emotion and cognitive control. In addition to developmental and cognitive factors, the DMC account also predicts that relative engagement in proactive versus reactive control can be influenced by affective and motivational factors, particularly due to the role of dopaminergic projections to the prefrontal cortex (Braver, 2012; Braver et al., 2007). This prediction has been tested in various ways and has led to a recent surge of studies revealing how emotional affect and stimuli interact with proactive and reactive control. Although the overwhelming majority of these studies has been conducted with younger adults, the results of some of these studies on emotion interactions are informative to review in order to elucidate the various ways emotion can interact with the two control modes. For example, Dreisbach (2006) presented younger adults with a positive, negative, or neutral picture (between-subjects) prior to the start of each trial of the AX-CPT task. Results across two experiments revealed reduced proactive coupled with increased reactive control in the group that received positive pictures, relative to those that received neutral or negative pictures. Interestingly, in Experiment 2, the paradigm was modified so that distracter letters were added during the delay between cue and probe presentation to increase task demands. With this modification, the authors again found reduced proactive and even more evident patterns of increased reactive control (indexed by an increase in probe-lure errors) as compared to Experiment 1. The authors attributed exaggeration of the detrimental effect of positive pictures to increased maintenance demands, with greater distractibility under conditions with positive pictures. This finding of reduced proactive control under conditions in which positive pictures preceded each trial has also been replicated with a response cueing paradigm (Fröber \& Dreisbach, 2012) and again with the AX-CPT task (Fröber \& Dreisbach, 2014). 
Studies have also attempted to induce different affective states prior to the AX-CPT task to examine the effects of induced mood on control patterns. For example, one study induced younger adults into a positive or neutral mood using video clips (van Wouwe, Band, \& Ridderinkhof, 2011). Behavioural results showed reduced proactive control under positive mood, with slower and less accurate responses on cue-lure trials compared to the other trial types, but no changes in reactive control. Results from event-related potentials collected during task completion were also consistent with the behavioural results. Conflicting results have been found though, as one similar study with younger adults observed slight increases in proactive control under positive relative to neutral mood inductions (Chiew \& Braver, 2014).

Overall, studies have primarily contrasted positive and neutral emotions (using mood inductions or emotional stimuli), with some research using negative stimuli (e.g., Dreisbach, 2006) but no studies examining the effects of induced negative moods on cognitive control. The results from the studies reviewed in this section have demonstrated the interactive relationship between cognitive control and emotion. There appears to be fairly consistent evidence that positive, relative to neutral (Fröber \& Dreisbach, 2014) and negative (Dreisbach, 2006; Fröber \& Dreisbach, 2012), images presented on a trial-by-trial basis can have detrimental effects on proactive control in younger adults. A similar detrimental effect to proactive control has been found from positive compared to neutral mood inductions prior to the start of the task (van Wouwe et al., 2011), but conflicting findings of enhanced proactive control under similar conditions have also been found (Chiew \& Braver, 2014). Studies have not found evidence that negative pictures can affect cognitive control (Dreisbach, 2006; Fröber \& Dreisbach, 2012). This suggests that negative emotion may not have the same moderating effects as positive emotion. However, mood measures were not administered in these studies, so is not clear what influences, 
if any, these images may have had on participants' mood. Thus, research examining the effect of induced negative moods on cognitive control would contribute to this literature.

Most strikingly, studies on emotion-control interactions thus far have been conducted with younger adults, with a lack of research on older adults. The closest to informing this interaction is a study examining motivational manipulations by Schmitt, Ferdinand, and Kray (2015) with older and younger adults ${ }^{3}$. This study compared the effect of cues that indicated an upcoming reward or penalty versus neutral (no reward or penalty) trial on a modified AX-CPT task with younger and older adults. Results indicated that proactive control in older adults was enhanced from both gain and penalty cues, relative to neutral ones. This demonstrates that increasing the motivational (and possibly emotional) elements of cues can enhance proactive control in older adults. However, it is not clear how these motivational tendencies relate to emotional influences, especially considering the complex nature of emotion-motivation distinctions (Chiew \& Braver, 2011).

Considering evidence that positive emotions can hinder proactive control in younger adults, this area of research may be a valuable one to study in older adults, as it could provide insight into potential factors that moderate older adults' proactive control difficulties. This is especially important considering evidence that older adults have typically been found to experience more positive moods than younger adults (e.g., Isaacowitz, Toner, Goren, \& Wilson, 2008; Knight et al., 2007; Mather \& Knight, 2005; Noh et al., 2012) and they have preferences toward positive and away from negative information (Reed, Chan, \& Mikels, 2014).

\footnotetext{
${ }^{3}$ This study was reviewed to demonstrate that younger and older adults differ on cognitive control due to a motivational manipulation. However, there is evidence to suggest that emotion and motivation exert different influences on control (Chiew \& Braver, 2014). A thorough discussion of the differences are beyond the scope of this dissertation (but see Chiew \& Braver, 2011 for further insight).
} 
Summary. Three conclusions can be made based on the DMC framework and the review of its predictions and supporting research: (1) there is evidence of two control modes - one characterized by continuous maintenance of task goals (proactive) and another that relies on reactivation of task goals when needed (reactive); (2) older adults' cognitive control deficits appear to be specific to decreased engagement of proactive control, presumably due to agerelated limitations on processing resources; and (3) shifts between the two control modes are amenable to external factors, such as task strategy training, reduced cognitive demands of the task, and emotional manipulations. However, research in this area is relatively new and there are still many unaddressed questions. Most notably, review of the research conducted thus far suggests that there is potential to shift older adults' control strategies (Paxton et al., 2006). An open question remains: could emotional manipulations - similar to those that have been conducted with younger adults - also influence patterns of reactive and proactive control in older adults? If so, would the effects differ in the two age groups? A plethora of studies has suggested that younger and older adults have different motivational inclinations that result in divergent emotional goals and preferences. These age-related differences in emotional patterns have been found in studies examining various cognitive abilities, including cognitive control. The following review will outline these age-related emotional differences, which support the possibility that interactions between cognitive control and emotion may differ across the life span.

\section{Aging, Emotion, and Cognitive Control}

In contrast to age-related cognitive control declines, older adults demonstrate evidence of preserved emotional regulation and processing (Reed \& Carstensen, 2012). The socioemotional selectivity theory (SST; Carstensen, Isaacowitz, \& Charles, 1999) provides an explanation as to 
why emotional functioning is relatively intact, in face of declines in other domains. Studies have also found that cognitive control can impact these processes, as reviewed later in this section.

Socioemotional selectivity theory. The central tenet of the socioemotional selectivity theory (SST; Carstensen et al., 1999) is that perceived time constraints cause one to shift away from future-oriented and towards present-oriented goals instead. Specifically, this theory predicts that expansive time horizons cause prioritization of future-oriented goals related to information or knowledge acquisition. In contrast, limited time horizons cause prioritization of presentoriented goals related to achievement of emotional well-being. As advancing age often results in perceptions of greater constraints on time horizons, this theory is frequently used to explain agerelated changes in emotional regulation and processing. Thus, findings of more effective regulation of emotions and preserved emotional processing in older, relative to younger, adults is attributed to motivational goal shifts toward prioritization of positive well-being in advanced age (Charles \& Carstensen, 2010). The SST presumes that these emotional goals are chronicallyactivated in older adults, and may explain why age differences have been found in reported moods and mood regulation. For example, in a longitudinal study spanning 10 years, Carstensen et al. (2011) found that aging is associated with greater overall emotional regulation and wellbeing. This is consistent with predictions from the SST that older adults prioritize emotional well-being. Cross-sectional studies consistently reveal that older adults self-report more positive moods than younger adults (e.g., Isaacowitz, Toner, Goren, \& Wilson, 2008; Knight et al., 2007; Mather \& Knight, 2005; Noh et al., 2012). Older adults are also efficient at regulating out of negative moods so that they achieve positive moods faster than younger adults (Larcom \& Isaacowitz, 2009). 
Furthermore, older adults' motivational inclinations toward achieving positive mood are hypothesized to be implicated in findings of age differences in the effects of emotional stimuli and mood on attention and memory tasks. Specifically, older adults' chronic emotional goals are hypothesized to result in enhanced processing towards information that can help them achieve positive mood (e.g., positive stimuli). This is often interpreted as reflecting an age-related “positivity” bias (Carstensen \& Mikels, 2005; Mather \& Carstensen, 2005). In contrast, older adults avoid negative stimuli (i.e., an “anti-negativity” bias), again due to the potential threats they pose to positive mood (Mather \& Carstensen, 2003). This relative shift away from negative, and toward positive material in older adults (Mather \& Carstensen, 2005) has been found in studies of attention (e.g., Isaacowitz, Toner, \& Neupert, 2009; Mather \& Carstensen, 2003) and long-term memory (Charles, Mather, \& Carstensen, 2003) and is typically termed the "positivity effect” (Mather \& Carstensen, 2005). For example, studies have found that older adults allocate more visual attention toward positive and away from negative stimuli, relative to younger adults (Isaacowitz \& Noh, 2011; Isaacowitz, 2012; Knight et al., 2007). However, findings of the positivity effect are not always consistent (Murphy \& Isaacowitz, 2008), with some studies finding no evidence of a preference toward positive information. This inconsistency was addressed by a recent meta-analysis that found a robust positivity effect across 100 studies of attention and memory (Reed et al., 2014). Results revealed that the positivity effect is larger when older adults are allowed the opportunity to freely and naturally engage with the emotional materials, such as in open-ended encoding conditions (e.g., viewing slideshow of pictures as if watching television; Charles et al., 2003), without many constraints imposed by specific task instructions (Reed et al., 2014). Finally, studies directly assessing mood have found that older adults' propensities toward positive moods are related to certain aspects of attention and that 
these relationships manifest in different ways in younger adults (e.g., Noh, Larcom, Liu, \& Isaacowitz, 2012; described later in this section).

Of particular interest to this dissertation are findings that older adults appear to recruit cognitive control in order to implement their positivity preferences. Mather and Knight (2005) found evidence across three studies that cognitive control may contribute to older adults’ ability to better remember positive versus negative pictures in memory. For example, older adults who performed well on tasks involving cognitive control were more likely to remember positive versus negative pictures than those who scored low on these tasks. Relatedly, another study found that divided attention can eliminate, and even reverse, the positivity effect (Knight et al., 2007). In this study, older adults under divided attention conditions (i.e., completing two concurrent attention tasks intended to reduce attentional or cognitive resources) did not show positivity effects. Instead, they allocated more visual attention toward negative than positive items, demonstrating a "reversal" of the positivity effect compared to those under full attention conditions (who attended more toward positive items). Results from these studies have led researchers to suggest that older adults appear to be motivated by their emotional goals to recruit cognitive control processes to help enhance positive and diminish negative information in attention and memory (Mather \& Knight, 2005). When resources are taxed, older adults are presumably no longer able to direct cognitive control toward the implementation of their emotional goals, which results in reduced positivity biases. These studies provide insight into the interactions between emotion and cognitive control in older adults.

Further insight into these interactions can be gained by examining how emotion affects age differences in cognitive control. Although abundant work has examined age differences in the effects of emotion on attention and memory performance, research has not yet examined 
whether emotion affects age differences in proactive or reactive control specifically. However, research on emotion effects on component processes that are postulated to contribute to cognitive control, such as goal representation/utilization, maintenance of information in working memory, task-switching and updating, response preparation, and interference resolution (Braver \& Barch, 2002; Braver et al., 2007) may be informative. The following sections will review how these components are affected by emotion in different ways for older than younger adults and the review will be categorized by two types of effects: mood effects (i.e., influence of internal mood states of participants) and emotional stimuli effects (i.e., the influence of emotional stimuli in the paradigm/task). This is an artificial distinction for the purpose of clarity in this dissertation and has the utility of helping to identify whether patterns are similar or different for effects of mood versus emotional stimuli. In real-life situations, the distinction is not easily disentangled. For example, the use of emotional stimuli in a cognitive task are likely to elicit transient mood effects that are congruent with the valence of the stimuli. Current moods could also impact how stimuli are attended to. Experiencing a particular mood, such as a sad mood, may make one seek out positive stimuli or information to help with emotional regulation (Isaacowitz et al., 2008). However, there are certain differences in studies that examine mood versus emotional stimuli effects. For example, studies that incorporate emotional stimuli do not always directly assess the impact of these stimuli on mood. Rather, they look at differences in processing of these stimuli (e.g., attention toward or memory for emotional stimuli). Thus, it is often unknown how participants' moods are influenced by the stimuli, rendering it difficult to elucidate the impact on performance of mood changes arising from these stimuli. In contrast, studies focusing on mood effects provide measurements of mood. Many of these studies examine the impact of mood on tasks containing neutral information (e.g., with numbers, letters, or neutral words; Biss, Weeks, 
\& Hasher, 2012). There are also studies that look at the interactions of mood effects on processing of emotional versus neutral stimuli (e.g., Isaacowitz et al., 2009), but such studies are relatively rare (Isaacowitz \& Blanchard-Fields, 2012). Thus, although the distinction is a fairly artificial one, it helps to disentangle the complex interactions between emotional stimuli and mood effects and provides the utility of more clear patterns of evidence. This distinction helps pinpoint emotional effects that may be driven primarily by external influences (e.g., how emotional items attract attention) versus those that are mostly affected by internal mood-based effects (e.g., the impact of induced mood on attention).

Effects of mood. A significant component of cognitive control is attention (Braver et al., 2007). Researchers have examined how reported levels of current mood state are related to attention and whether these relationships differ in younger and older adults. In one study by Noh et al. (2012), younger and older adults completed the Attention Network Test (ANT; Fan, McCandliss, Sommer, Raz, \& Posner, 2002), which is a task that provides separate indices of alerting (maintaining an alert state), orienting (orienting to spatial cues that direct attention to task-relevant information), and executive attention (resolving conflict from interference). Results indicated that higher levels of positive mood and lower levels of negative mood in older adults were associated with enhanced orienting towards cues. The researchers interpreted these results as suggesting that positive affect may specifically broaden attention in order to increase sensitivity to environmental cues, possibly in search of cues that could help maintain positive affect. Notably, this ability to efficiently use spatial cues (that are $100 \%$ predictive of the location of an upcoming target) to perform well on the ANT task is similar to the use of the predictive cues in the AX-CPT task and may inform hypotheses about the effects of positive mood on proactive control. In younger adults, no moderating effects of positive or negative 
moods were found, although positive correlations between negative mood and increased orienting were found. Thus, this study demonstrates that mood can have different effects on components of attention in older and younger adults.

In addition to examining the relationship between current mood states and cognitive performance, researchers have also examined effects more directly by experimentally inducing positive, negative, or neutral moods prior to completing a cognitive task (e.g., Biss et al., 2012; Phillips, Smith, \& Gilhooly, 2002). Generally, this consists of a mood induction procedure and a manipulation check that compares participants’ mood ratings before and after the induction. Mood inductions have been accomplished through a variety of procedures. For example, some studies have participants listen to music that is congruent with positive or negative moods (e.g., upbeat versus slow and melancholic music, respectively; Isaacowitz et al., 2009) or show images or video clips that portray positive or negative content (e.g., Phillips et al., 2002). Some procedures also combine these techniques (e.g., Biss et al., 2012). Studies typically report that mood inductions are generally successful at eliciting the desired mood state in participants (e.g., Isaacowitz et al., 2008). These inductions generate psychophysiological responses and activation of brain areas implicated in emotions (e.g., amygdala; Dyck et al., 2011) that are consistent with self-reports of $\operatorname{mood}^{4}$.

Although studies have not used mood inductions to examine emotion effects on proactive and reactive control in older adults, some studies have used inductions to examine influences on hypothesized components of cognitive control that can provide some insight into possible mood effects. For example, one study induced older adults into positive, negative, or neutral moods

\footnotetext{
${ }^{4}$ There are some conceptual and practical issues that need to be considered when using inductions to examine the influences of mood on cognition. These issues will be discussed in the General Discussion section.
} 
prior to the London of Tower task (Phillips et al., 2002). This task is often used to assess the ability to plan behaviour to accomplish the goal of moving discs into a particular arrangement and to do so using the minimum possible number of moves. Successful completion of this task requires advance preparation of responses, holding relevant information in working memory, and monitoring and adjusting behaviour - all of which recruit processes that are similar to those presumably recruited in proactive control as well (Braver et al., 2007). Results from this study revealed poorer performance under positive and negative relative to neutral moods and suggests impairments to planning arising from emotional mood states. A similar study found slightly different patterns with younger adults. Relative to neutral mood, younger adults also showed reduced planning ability from induced positive but not from negative mood (Oaksford, Morris, Grainger, \& Williams, 1996). Thus, there is some evidence of age differences in patterns of mood effects on planning. Phillips et al. (2002) suggested that older adults' may attempt to maintain positive moods or regulate out of negative moods that arise from the positive and negative inductions, respectively, and that these processes may have competed with task performance which resulted in planning decrements.

Studies have also examined the effects of mood on working memory, which is hypothesized to play a significant role in proactive control (Braver et al., 2007; Redick, 2014). In one study, younger and older adults were instructed to complete a 2-back working memory task while down-regulating a negative affect (i.e., disgust) that was generated from a mood induction prior to the task (Scheibe $\&$ Blanchard-Fields, 2009). This negative affect resulted in poorer working memory performance in younger, but not older, adults. The authors postulated that down-regulation of negative affect was possibly less resource-demanding for older adults, due to their abilities to effectively regulate emotions. Results from this study conflict with the 
hypothesis raised by Phillips et al. (2002) that older adults' cognitive performance is negatively impacted by mood regulation. A speculative explanation may be that older adults' only exhibit emotion effects when the task is more complex, as in the Tower of London task.

What about the effects of positive emotion on working memory? Carpenter et al. (2013) examined how a positive mood induction influenced working memory performance in older adults relative to neutral mood. Results indicated that positive mood facilitated working memory performance and also improved performance on a decision-making task (card choice with gains and losses). A study also found that positive mood in older adults can have a detrimental effect on inhibitory control, which is another important component of cognitive control (Braver et al., 2007). Biss, Weeks, and Hasher (2012) found that positive mood reduced older adults' inhibitory control over distraction from neutral words that were superimposed on images used in a 1-back working memory task. Interestingly, this reduced inhibitory control was evidenced by greater priming for the distracter words (as revealed on a subsequent implicit memory test), rather than reduced performance on the 1-back task. Thus, positive mood appeared to broaden attention (resulting in encoding of both task-relevant and task-irrelevant items), but not at the expense of working memory performance. A similar effect was also found in younger adults under induced positive mood (relative to neutral mood; Biss \& Hasher, 2011).

Finally, further insights arise from a study examining set-switching and inhibitory control in younger and older adults in which participants were induced into positive, negative, and neutral moods (Chu, 2014). The task consisted of cue-based switching tasks in which participants switched between responding based on different task rules (e.g., responding whether a figure was a triangle or rectangular in one task versus responding on the colour dimension). Age group comparisons showed that older adults showed deficits on switching and inhibitory 
control compared to younger adults but this was apparent only under induced neutral moods; age differences were not apparent under induced positive or negative moods. Additionally, results revealed that older adults' switching abilities benefited from the emotional relative to neutral inductions. In contrast, there was some evidence that younger adults' switching abilities were impaired by induced positive relative to negative and neutral moods. Thus, this study adds to the evidence of age differences in the effects of induced moods on components of cognitive control.

In summary, the effects of mood on various cognitive abilities appear to show differential patterns that vary by age, such as task-switching abilities (Chu, 2014). Within an age group, there were also inconsistencies in the influences (e.g., beneficial or detrimental) of mood on performance. In particular, there was evidence of facilitative effects of positive mood in older adults in more simple tasks involving attention (Noh et al., 2012) and working memory (Carpenter et al., 2013), but detrimental effects for more complex planning tasks (Phillips et al., 2002).

Effects of emotional stimuli. Traditionally, tasks measuring cognitive control use neutral stimuli, such as letters or numbers, to minimize the influence of potential confounding factors. However, researchers are increasingly incorporating more meaningful stimuli into cognitive tasks - both to study the effects of such stimuli on cognition and as an attempt to increase the external validity of these measures. Relevant to this review are studies using emotional stimuli in working memory tasks. For example, Borg et al. (2011) examined working memory in younger and older adults by incorporating emotional pictures in two working memory tasks. The first task - a visual working memory task - required participants to maintain four sequentially presented pictures (two negative and two neutral pictures) over a 4500 ms delay. A probe picture appeared after this delay and participants indicated whether it belonged to the current target set. For both 
age groups, maintenance of negative stimuli was better (higher accuracy) than maintenance of neutral stimuli. These results demonstrate that emotional stimuli can aid maintenance of target items in working memory, regardless of age group. However, differential effects of age appeared in the second task - a binding memory task. Similar to the first task, participants were presented with two negative and two neutral pictures sequentially. However, the goal in this task was to correctly indicate whether the probe picture (which always belonged to the current target set) was presented in the same location in which it had originally appeared. Thus, this task required participants to maintain both the picture and its visuospatial location. This was hypothesized to be more demanding than the first task, due to a higher working memory load which requires greater cognitive control. Results now indicated an age by valence interaction - younger adults performed equally for both negative and neutral pictures, whereas performance was lower for negative relative to neutral pictures in older adults. This suggested that as task demands increased, older adults were impaired by negative stimuli. Unfortunately, this study did not incorporate positive pictures, so it is unknown whether a similar pattern would be demonstrated for positive stimuli.

Insights into the maintenance and inhibitory control of positive stimuli in working memory come from a study by Truong and Yang (2014). Younger and older adults completed a delayed-response working memory task requiring both maintenance and inhibition of emotional (positive and negative) and neutral words. The maintenance of target information is considered to be less demanding and shows less age-related declines (Gazzaley, Cooney, Rissman, \& D’Esposito, 2005). In contrast, the inhibition of interfering distracters is more demanding and shows greater age-related declines (Gazzaley et al., 2005). In this task, four words were presented sequentially on each trial - two words were cued as to-be-remembered target words 
and two as to-be-ignored distracter words. Following a 500 ms delay, participants indicated whether a probe was a target word. Results indicated that both positive and negative targets, relative to neutral ones, facilitated working memory in both age groups, with slightly better performance for positive words. In contrast, emotional distracters disrupted performance, but this varied by age and valence. Younger adults were successful at rejecting negative distracters but this was not true for older adults, suggesting an age-related decline in the inhibitory control of interference from negative words. Thus, results from this study indicate how maintenance and inhibition of emotional information control can differ by age and valence.

Samanez-Larkin, Robertson, Mikels, Carstensen, and Gotlib (2009) also examined the effects of emotional stimuli on executive attention tasks in younger and older adults. This study compared performance on non-emotional versus emotional flanker tasks. In the non-emotional task, participants indicated whether a central target word was a metal or fruit. Interference resulted from incongruent flanking words (i.e., from a different category than the target word). For example, if the target word was a fruit, the incongruent flanking word was a metal. In the emotional task, the central word was either positive or negative and participants had to ignore interference from flanking words that were incongruent (i.e., opposite valence category). Results indicated that both age groups showed significant interference effects in the non-emotional task. However, only younger, but not older, adults showed interference on the emotional task. Thus, older adults appear to be better at resolving interference when emotional stimuli are used, regardless of valence. Although there was no evidence of an age-related positivity effect, the results indicated that both younger and older adults experienced more interference when the distracter flanking words were negative versus positive. This again suggests that negative 
information can have impairing effects on processes that are related to cognitive control, as was found in Borg et al. (2011) and Truong and Yang (2014).

Finally, emotional stimuli have been used in a working memory task with younger and older adults (Mikels, Larkin, Reuter-Lorenz, \& Cartensen, 2005). In contrast to the aforementioned studies in which a specific stimulus (or stimuli) had to be maintained over a delay period, participants were instructed instead to maintain the intensity of the emotional affect elicited by the stimulus. This was compared to a non-emotional working memory task in which maintenance of brightness intensities was required instead. Results were consistent with some of the studies already reviewed: older adults exhibited better working memory performance when asked to maintain emotional rather than (neutral) brightness intensities. Particularly interesting were findings that older adults outperformed their younger counterparts on trials involving maintenance of positive affect and older adults also exhibited better performance on positive than negative trials. In contrast, younger adults performed better on negative relative to positive trials. Thus, these results provide further evidence that emotional versus neutral information can exert different effects on working memory.

Together, the studies examining the effects of emotional stimuli on components of cognitive control show that the valence of emotional stimuli can have different effects and that these effects sometimes differ by age. The most consistent evidence was that negative stimuli, relative to positive and neutral stimuli, can result in poorer working memory or inhibitory control in older, but not younger adults (Borg et al., 2011; Samanez-Larkin et al., 2009; Truong \& Yang, 2014). Additionally, older adults' maintenance of positive affect in working memory appears to be better than for negative or neutral affect (Mikels et al., 2005). These results are somewhat consistent with the literature on older adults' positivity bias in attention and memory that have 
found better performance when tasks incorporate positive versus negative items. This provides some support for the idea that older adults may direct cognitive control toward positive and away from negative information (Knight et al., 2007; Mather \& Knight, 2005), which can enhance task performance when the task relevant stimuli are positive (e.g., Mikels et al., 2005).

Summary. The review on emotion and aging leads to four overall conclusions: (1) according to the SST, older adults prioritize emotional versus knowledge/information goals due to motivations to achieve emotional well-being; (2) these motivations often lead to enhanced processing of positive and/or diminished processing of negative versus neutral material (or "positivity effects"); (3) the enhanced processing of emotional materials (positive specifically) appears to require cognitive control (Mather \& Knight, 2005); and (4) mood states and emotional stimuli can have both facilitative and disruptive effects on components of cognitive control and these effects seem to vary by valence and age. In particular, there was consistent evidence that positive moods and stimuli can aid aspects of older adults' cognitive control (e.g., Carpenter et al., 2013; Mikels et al., 2005). In contrast, negative moods and stimuli (relative to neutral) can be facilitative under conditions of reduced task demand (e.g., Borg et al., 2011), but disruptive when demands are higher, such as in inhibitory control (e.g., Samanez-Larkin et al., 2009; Truong \& Yang, 2014). These patterns are not always observed in younger adults (e.g., Chu, 2014) and are also somewhat inconsistent with findings of reduced cognitive control in younger adults due to positive versus neutral mood or stimuli (e.g., Dreisbach, 2006; van Wouwe et al., 2011). Together, these results further suggest divergent patterns of emotional effects on cognitive control across the life span. These interactions between cognitive control, aging, and emotion will be examined in this dissertation. The next chapter will present an overview of the objectives, rationale, and overarching hypotheses. 


\section{Chapter 2: Overview of the Study}

\section{Objectives}

The literature review on cognitive control, aging, and emotion has demonstrated several patterns that are relevant to the current study: (1) age-related cognitive control deficits appear to be specific to proactive goal maintenance, according to the dual mechanisms of control framework; (2) in contrast to cognitive control declines, older adults are effective at regulating their emotions and demonstrate preserved processing of emotional stimuli, thus experiencing greater overall positive well-being than younger adults; and (3) the growing literature on cognitive control, aging, and emotion interactions suggests that emotional stimuli and motivation driven by emotional regulation goals (to achieve positive affect) may have the potential to moderate or even enhance performance on cognitive control related tasks.

These conclusions lead to the following research question: Could cognitive control patterns in older adults be modulated through the manipulation of emotional affect and the incorporation of salient and emotional stimuli? To address these research questions, three experiments were conducted to examine the effects of induced emotional affect (Experiments 1 and 2) and emotional task stimuli (Experiment 3) on cognitive control patterns in younger and older adults. The detailed objectives, rationale, and hypotheses for each experiment will be presented in the following chapters, but a general overview of them are presented here first.

\section{Experiments}

As the experiments were set within the DMC framework, the basic AX-CPT paradigm served as the foundation of the tasks used in all three experiments. This allowed for comparisons with existing research on proactive versus reactive control. In addition, this task has previously demonstrated its ability to be readily modified for examination of different variables of interest 
(e.g., motivation; Braver et al., 2009; Locke \& Braver, 2008) and it also correlates with more traditional neuropsychological measures, such as the trail-making test (Braver et al., 2005).

Specifically, participants received neutral (Experiment 1A) or negative (Experiment 1B) mood inductions prior to completing the standard AX-CPT task (Cohen et al., 1999). In Experiment 2, participants received neutral (Experiment 2A) or emotional mood inductions (Experiment 2B), prior to completing a modified AX-CPT task with faces of different genders as cues and letters as probes. Experiment 2A piloted the modified AX-CPT used in Experiment 2B with the main goal of reducing cognitive demands to promote the emergence of emotional effects. Experiment 2B examined the effects of negative and positive mood inductions on performance on this modified face-letter AX-CPT task. The purpose was to examine whether effects of mood inductions would be influenced (e.g., become more or less evident) by an AXCPT version with reduced task demands. Finally, Experiment 3 consisted of an AX-CPT task that used emotional and neutral faces as cues and probes and was conducted with older adults only. The emotional expressions of the cue stimuli were manipulated to examine the effects of emotional and salient face stimuli while still maintaining the basic structure of the standard AXCPT task. It served to address the question of whether older adults' preference towards positive information could have an advantageous effect on proactive control with socially salient face stimuli. The next chapter (General Methods) will outline methodology that is the same or similar across all experiments.

\section{Hypotheses}

Several hypotheses were derived based on propositions from the DMC framework and the SST, as well as the relevant empirical research conducted thus far. A more detailed description of the specific hypotheses for each experiment will be presented in the respective 
sections. In Experiment 1, it was hypothesized that negative, relative to neutral, mood inductions would have differential effects on older and younger adults. In Experiment 2, it was hypothesized that the effects of the mood inductions on cognitive control patterns may be more apparent, or more likely to emerge, when assessed with an easier AX-CPT version. Finally, Experiment 3 tested the hypothesis that older adults' positive preferences will cause them to focus more on face cues displaying positive and less on those displaying negative emotions, relative to neutral emotion. This would subsequently have the effect of enhancing proactive control, as greater cue-related processing was expected to occur for positive face cues. 


\section{Chapter 3: General Methods}

Similar methods were used across all three experiments of this dissertation. This section describes these common procedures. Details on variations that are specific to an experiment are outlined in the respective Method section for each experiment.

\section{Participants}

Sample size. A-priori power analyses were conducted using program G*Power 3.1 (Erdfelder, Faul \& Buckner, 1996) to determine the necessary sample size for each experiment with power (1- $\beta$ ) at .95 and $\alpha=.05$. For Experiments 1 and 2, results indicated that a sample size of at least $n=23$ per age by mood induction group would be required to detect a small-medium effect size $(f=0.20)$ using the “ANOVA: Repeated measures, within-between interaction” option. For Experiment 3, a sample size of at least $n=22$ would be required to detect a medium effect size $(f=0.25)$ using the “ANOVA: Repeated measures, within factors” option.

Inclusion criteria. All participants were healthy younger (ages 18-29) and older adults (ages 65 and greater). Participants were excluded from analysis if they had: (a) previous neurological disorders (stroke, dementia, prolonged periods of unconsciousness greater than five minutes, and major head injury); and (b) uncontrolled medical conditions (e.g., diabetes, cholesterol, and cardiovascular diseases); (c) current diagnoses of mood disorders (e.g., depression or anxiety disorders). Older adults were excluded if they scored greater than 6 on the Short Blessed Test (SBT; Katzman et al., 1983), suggesting dementia-related cognitive impairments. Participants were also excluded if they received scores equal or greater than 28 on the depression scale and greater than 20 on the anxiety scale of the Depression, Affect, and Stress Scale (DASS; Lovibond \& Lovibond, 1995). Scores in these ranges were indicative of “extremely severe” experiences of depression and anxiety symptoms within the past week. 
Recruitment. All younger adults (Experiments 1 and 2) were recruited from an undergraduate participant pool of Introductory Psychology students at Ryerson University and received 1.5 course credits as incentive for a 1.5-hour session. Older adults in all experiments were recruited from the Ryerson Senior Participant pool, which is an older adult participant pool maintained by the Department of Psychology at Ryerson University. Participants who took part in one of the three experiments did not participate in any of the other experiments. Older adults received a \$20 cash incentive for a 2-hour session in Experiments 1 and 2 and \$12 in Experiment 3 for a 1-hour session. All participants were tested at the Cognitive Aging Laboratory at Ryerson University. All tasks and procedures were reviewed and approved by the Ryerson Research Ethics Board (REB protocol \#: 2013-286-1; Appendix I) to ensure compliance with experimental guidelines for research involving human participants.

\section{Task Administration}

All AX-CPT tasks were programmed and administered with E-Prime 2.0 software (Psychology Software Tools, Pittsburgh, PA). Participants in Experiments 1 and 2 completed all computer tasks on a 17" laptop with an external 19" monitor display set at a resolution of $1440 \times$ 900 pixels. They used an external keyboard and mouse to input their responses. Participants in Experiment 3 used the laptop's keyboard and screen set at a resolution of $1920 \times 1080$ pixels.

\section{AX-CPT Tasks}

Variations of the AX-CPT task were used across all experiments to examine reactive and proactive control. All variants followed the same basic task rule (i.e., respond "target" to a valid probe following a valid cue). Experiment 1 used the standard letter AX-CPT (Cohen et al., 1999), which consisted of letters as cue and probe stimuli. Experiment 2 used the face-letter AXCPT which consisted of faces as cues and letters as probes. Experiment 3 used only faces as both 
cue and probe stimuli. Detailed descriptions of each variant are detailed in the Method section for each experiment.

\section{Mood Induction and Ratings}

Mood inductions. Mood inductions were used in Experiments 1 and 2 in an attempt to manipulate mood states, prior to completing the AX-CPT task. Each mood induction consisted of a slideshow of 30 pictures (positive, negative, or neutral in emotional valence) selected from the International Affective Picture System (IAPS; Lang, Bradley, \& Cuthbert, 2008). This procedure was similar to that used in other studies with younger and older adults (Biss \& Hasher, 2011; Biss et al., 2012) that were successful at inducing the intended moods. IAPS contains pictures that have been rated for emotional valence and arousal on a 9-point scale, with lower scores representing negative valence/low arousal and high scores representing positive/high arousal. All pictures that were selected were low in arousal (arousal ratings $<5.50$ ), as the goal was to induce low arousal moods. This allowed for a better match of the arousal levels across the different valence conditions (e.g., positive/negative vs. neutral). Analyses on the valence ratings revealed significant differences between all conditions in the expected direction, with the lowest ratings in the negative, followed by the neutral, and the positive condition, $p s<.001$. Arousal ratings for the negative and positive induction pictures did not differ, $p=.980$, and both were rated higher in arousal than the neutral induction pictures, $p s<.001$. Table 1 details the mean valence and arousal ratings of the selected pictures. The pictures selected for each induction were also approximately equated for the number of pictures containing humans, animals, and scenery. Images that displayed salient human facial expressions were avoided. 
Table 1

Characteristics of Mood Induction Pictures

\begin{tabular}{|c|c|c|c|c|c|c|}
\hline \multirow[b]{2}{*}{ Mood Induction } & \multicolumn{3}{|c|}{ Valence } & \multicolumn{3}{|c|}{ Arousal } \\
\hline & $M$ & $(S D)$ & Range & $M$ & $(S D)$ & Range \\
\hline Neutral & 5.34 & $(0.51)$ & $4.06-5.96$ & 4.00 & $(0.60)$ & $3.26-5.36$ \\
\hline Negative & 3.12 & $(0.47)$ & $2.12-3.94$ & 4.60 & $(0.49)$ & $3.52-5.50$ \\
\hline Positive & 7.16 & $(0.52)$ & $6.15-8.34$ & 4.60 & $(0.52)$ & $3.55-5.46$ \\
\hline
\end{tabular}

Each picture was presented centrally on the screen for five seconds. The slideshow of pictures was accompanied by mood-congruent sound clips free of verbal material: calm classical music for positive, melancholic classical music for negative, and ambient street noise for neutral mood inductions. The following clips were used: Carmen Suite, Intermezzo (positive); Peer Gynt, Aase's Death (negative); ambient street noise (neutral). The classical music clips were used because they have been validated for their ability to elicit subjective and psychophysiological reactions that are consistent with positive and negative moods (Russo, Vempala, \& Sandstrom, 2013). The ambient street noise has been validated in the Urban Soundscape Project as effective at inducing neutral moods (Loria \& Russo, 2012; www.torontosoundmap.com). Participants listened to the sound clips via Sennheiser HD280 Pro headphones. The entire slideshow lasted 2.5 minutes and looped once (in a different presentation order) for a total induction duration of 5 minutes. Participants underwent the inductions just before starting the first block of AX-CPT trials and this was repeated again before the second block.

Mood ratings. To assess changes in mood state arising from the inductions, participants rated their current mood state at six different time points throughout the task. These mood ratings occurred before (i.e., pre-induction 1, pre-induction 2) and after each of two mood inductions (i.e., post-induction 1, post-induction 2), as well as after each of the two blocks of AX-CPT trials (i.e., post-block 1, post-block 2). Two versions of the Self-Assessment Manikin (SAM; Bradley 
\& Lang, 1994) were used: one to assess the valence and another the arousal level of participants' current mood state. This consisted of nine figures depicting various emotional states with a corresponding number rating; lower numbers represented sad/low arousal and higher numbers represented happy/high arousal states. Participants responded by pressing the corresponding number key (Figure 2).
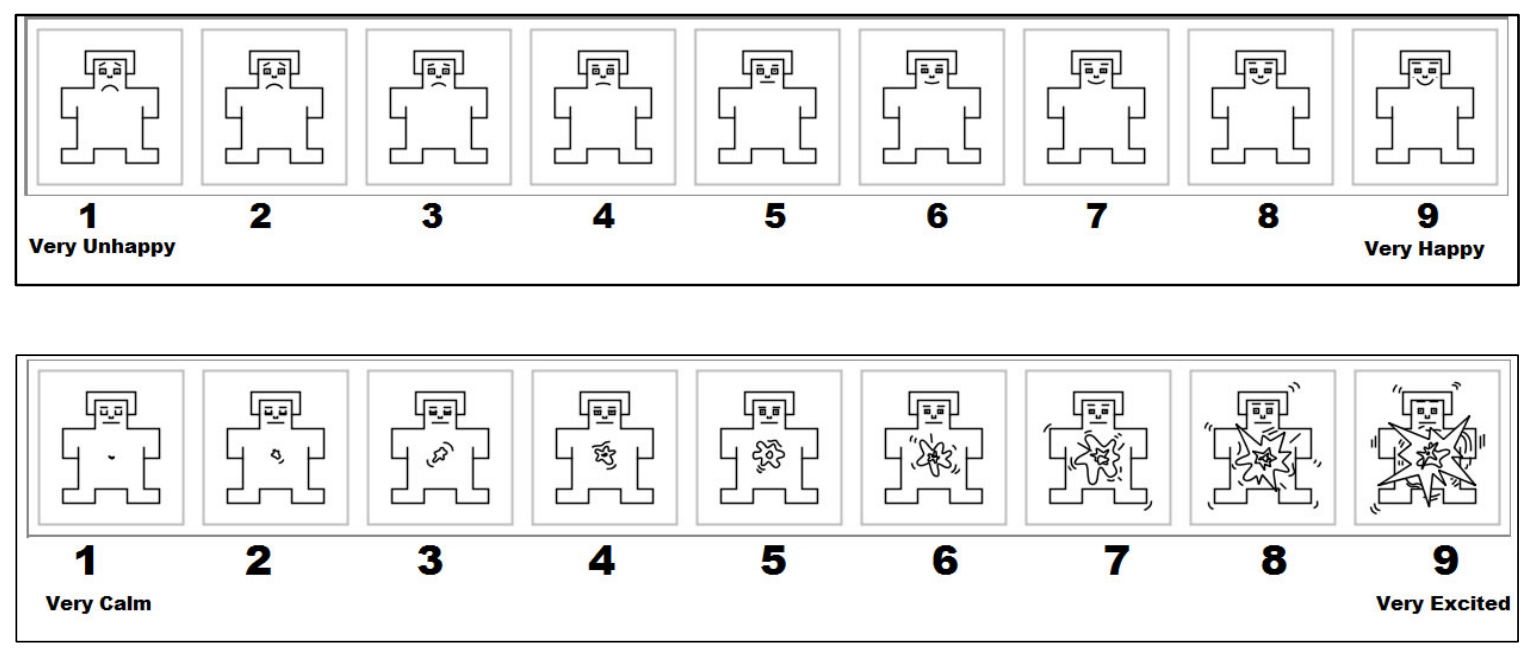

Figure 2. Self-Assessment Manikins (SAM); Upper panel: Valence ratings; Lower panel: Arousal ratings

Mood induction questionnaire. Participants were asked several questions pertaining to the mood induction (Appendix IX). The first question asked participants how familiar they were with the music (emotional induction groups) or sounds (neutral induction groups) they heard during the induction. The second question asked participants to rate their familiarity with classical music (emotional induction groups only). The third question asked participants to indicate the type of processing they engaged in while viewing and listening to the slideshow (e.g., actively paying attention to the whole slideshow versus the music/pictures only).

Responses were categorized into three types of processing: attention (responses 1-3), memorize (responses 4-6), or neither attention/memorize (response 7). The final question asked participants 
to rate how effective the slideshow was at inducing the specified mood state on a Likert scale from 1-9, with higher ratings indicating greater effectiveness.

\section{Individual Differences Measures}

Several questionnaires and cognitive measures were administered in all experiments (unless specified otherwise) to collect data on time perspective, affect, cognitive impairment, and demographic and health information. These questionnaires and measures were collected to assess for individual and group differences.

\section{Questionnaires.}

Future Time Perspective Scale (FTP). The FTP (Carstensen \& Lang, 1995) is a 10statement questionnaire that measures perceptions of time horizons. Participants indicate how true each statement is for them on a 7-point scale, with 1 representing "very untrue” and 7 representing “very true”. An example statement is: "My future is filled with possibilities”. Statements 8-10 are reverse-coded. The range of scores is 10-70; higher scores represent more expansive time perspectives. This scale was administered to examine for typical age-related patterns (i.e., expansive versus limited time horizons in younger and older adults, respectively).

Positive and Negative Affect Schedule (PANAS). The PANAS (Watson, Clark, \& Tellegen, 1988) is a 20-item questionnaire that assesses levels of self-reported positive (e.g., “excited”) and negative (e.g., “distressed”) affect at the moment of assessment. Ratings on a 5point scale range from 1 for "very slightly or not at all” to 5 for “extremely”. Responses on ten items (items 1, 3, 5, 9, 10, 12, 14, 16, 17, 19) are summed to calculate a positive affect score. Responses on the other ten items (items 2, 4, 6, 7, 8, 11, 13, 15, 18, 20) are summed to calculate a negative affect score. Scores for each affect range from 10-50, with higher scores indicating greater affect in that category. This questionnaire was included to distinguish between positive 
and negative mood states that participants were currently experiencing. It is frequently administered in studies examining emotion and aging and typically reveals age group differences, with older adults reporting lower negative affect and higher positive affect than younger adults (e.g., Isaacowitz, Toner, Goren, \& Wilson, 2008; Knight et al., 2007; Mather \& Knight, 2005; Noh et al., 2012).

\section{Depression, Anxiety, and Stress Scales (DASS21). The DASS21 (Lovibond \&} Lovibond, 1995) is a 21-item scale that measures and categorizes symptoms of depression (e.g., "I felt down-hearted and blue”, anxiety (e.g., "I felt I was close to panic”), and stress (e.g., "I found it difficult to relax”) experienced over the past week. The rating scale ranges from 0 (“did not apply to me at all”) to 3 (“applied to me very much, or most of the time”). Items 3, 5, 10, 13, 16, 17, 21 reflect depression symptoms; items 2, 4, 7, 9, 15, 19, 20 are anxiety symptoms; and items $1,6,8,11,12,14,18$ are stress symptoms. The responses to each category of symptoms are summed and multiplied by two for a category-specific score. The scores in each category range from 0-42, with higher scores representing more severe symptoms. Scores can be further categorized into different levels of symptom severity (normal, mild, moderate, severe, extremely severe) depending on the range the scores falls under. Participants with scores falling in the “extremely severe" categories of depression $(\geq 28)$ and anxiety $(\geq 20)$ were excluded in all experiments. The DASS21 was administered to examine for individual and group differences in recent mood states.

Background Information Questionnaire. Demographic (e.g., age, gender, years of education) and health information (e.g., history of neurological or psychological disorders, medications) were collected on a background information questionnaire. 
Cognitive tests. Several tests were administered to screen for dementia-related symptoms and to assess for individual and group differences on measures of visuospatial short-term memory, speed of processing, vocabulary, attention, and task-switching.

Short Blessed Test (SBT). The SBT (Katzman et al., 1983) is a 6-item test that checks for dementia-related cognitive impairments in orientation, memory, and concentration. The questions are administered orally and participants’ responses are recorded by the experimenter. The range of scores is 0 -28. Scores equal to or greater than 6 suggest possible dementia-related cognitive impairment and was used as a cut-off score for exclusion of participants from data analyses. This test was only administered to older adults.

Corsi block. The Corsi block task is a visuospatial short-term memory test administered on the computer. In this task, participants see nine grey squares, some of which briefly flash black. Participants are asked to click on the squares in the same sequence in which they turned black. The set size ranged from 2-3 squares in the practice trials and increased incrementally from 4-7 squares in the experimental trials (3 trials per set size). There were six practice and 12 experimental trials. Feedback was not provided. Following Rowe, Hasher, and Turcotte (2009), overall accuracy performance was calculated as the proportion of trials that were correctly recalled in the right sequence.

National Institutes of Health (NIH) Toolbox. The NIH Toolbox (Northwestern University and the National Institutes of Health, 2012) is a collection of standardized and validated computerized neuropsychological measures. The measures have been normed and validated in a large and diverse sample of participants ages 3-85 in the United States. All NIH Toolbox measures were administered on the computer using Internet Explorer 9.0 with highspeed cabled internet at 100 mbps, as per toolbox standardization requirements. All stimuli were 
displayed on an external 19” monitor and responses were collected via an external keyboard/mouse, which were all connected to the laptop. Four measures were selected from the cognition category of this toolbox to assess processing speed, vocabulary, attention, and taskswitching abilities. All tasks consisted of instructions and a practice block with accuracy feedback, followed by the experimental block. Participants completed the tasks in the order in which they are presented below.

Pattern Comparison Processing Speed Test. Pattern comparison is a measure of processing speed in which participants identify, as quickly as possible, whether two pictures are the same or different. They are given 90 seconds to complete this, up to a maximum of 130 items. This task was used to assess for typical age-related slowing. Scores range from 0-130, with higher scores representing faster processing speed. The score is based on the number of items correctly identified.

Picture Vocabulary Test. This test measures receptive vocabulary. Participants hear a word presented through the computer speakers and are asked to click on one out of four pictures that best represent the word. Administration of this task is adaptive to performance (i.e., the task becomes harder if the participant responds correctly or vice versa). Participants complete 20-30 trials within four minutes. This test provides a measure of crystallized intelligence, as indexed through level of vocabulary. Scores begin at 200, with higher numbers representing higher levels of vocabulary, and is calculated using item response theory.

Flanker Inhibitory Control and Attention Test. The Flanker test measured attentional/inhibitory control was measured with 20 trials lasting approximately three minutes. In this task, participants see a set of five arrows and are instructed to indicate the direction that the middle arrow is pointing. In congruent trials, the middle arrow points in the same direction as the 
rest of the arrows; for incongruent trials, the middle arrow points in the opposite direction. This test provides a measure of attention and inhibitory control and was used to assess for age differences. The scores are calculated based on a combination of accuracy and response time and range from 0-10, with higher scores representing greater attentional abilities.

Dimensional Change Card Sort Test (DCCST). DCCST is a measure of cognitive flexibility used for switching between different stimulus domains (i.e., task-switching). Participants are randomly cued to switch between matching pictures based on either the shape (a ball or truck shape) or colour (yellow or blue) dimension. Participants complete 30 trials in approximately four minutes. The scores range from $0-10$ and the same scoring process as the Flanker test is used.

\section{Data Analysis}

All analyses were conducted using SPSS 22. The statistical significance level was set at $p$ $=.05$ unless otherwise specified. The statistical significance level was adjusted for any post-hoc multiple comparisons with conservative Bonferroni corrections to reduce the chance of Type I errors. All correlations were reported as Pearson's coefficients. Partial eta squared effect sizes were reported. Conservative Greenhouse-Geisser corrections were adopted to adjust degrees of freedom if Mauchly's Test of Sphericity was significant $(p<.05)$, suggesting violation of the assumption of sphericity.

Data processing and analysis steps for certain tasks were the same in each experiment and are described together in the following section for conciseness.

Mood ratings. As a manipulation check and to examine age differences in any mood changes across the neutral inductions and the AX-CPT task, the SAM ratings collected at six time points were submitted to a 2 (Age: younger, older) $\times 6$ (Rating time: pre-induction/baseline, 
post-induction 1, post-block 1, pre-induction 2, post-induction 2, post-block 2) for valence and arousal ratings separately. Several a priori contrasts were specified: (1) differences between preinduction 1 (i.e., baseline) compared to the subsequent rating times, (2) pre-induction versus post-induction time points, (3) post-induction 1 versus post-induction 2. All post-hoc comparisons were evaluated at $p=.006$ (Bonferroni correction for eight post-hoc comparisons: 3 comparisons between post-induction 1 versus subsequent time points, 3 comparisons between post-block 2 versus subsequent time points, and 2 comparisons between pre-induction 2 and post-induction 2 versus post-block 2).

AX-CPT task performance. The dependent variables of primary interest were error rates and response times to probes for each of the four trial types (target, control, probe-lure, cuelure). Cues always required non-target responses - irrespective of trial type - and were included to ensure encoding of cue stimuli. Following standard practice (e.g., Braver et al., 2009), responses to cues were not analyzed as these responses are not informative about proactive or reactive tendencies. Error rates to probes were calculated as (1 - accuracy rate) and includes both incorrect and omitted/no response trials. For response time analyses, only response times (RTs) to correct trials were included; the percentage of trials included in each experiment were reported in the respective Results section. Following standard practice when comparing age groups on AX-CPT performance (e.g., Paxton et al., 2008, 2006), z-transformed RTs were used. A ztransformation was applied to RTs for each participant using his/her global mean and standard deviation for correct responses. The medians of the linearly-transformed RTs for each trial type 
were analyzed. The z-transformed median RTs controlled for individual differences and general age-related slowing ${ }^{5}$.

Separate analyses were conducted for target and non-target trials on the error rates and ztransformed median RTs. For target trials, ANOVAs were conducted to compare performance across age and mood induction groups (Braver et al., 2001, 2005). For non-target trials, 2 (Age: younger, older) $\times 3$ (Trial type: control, probe-lure, cue-lure) mixed ANOVAs were conducted. A priori contrasts were conducted to examine hypothesized age differences in probe-lure and cue-lure trials, which index reactive and proactive control, respectively. Specifically, independent samples $t$-tests were conducted to compare younger and older adults’ performance on cue-lure and probe-lure trials. Additionally, paired samples t-tests were conducted to examine performance differences between probe-lure versus cue-lure trials, within each age group separately.

To provide a composite measure of proactive control, two proactive indices were calculated using errors and median correct RTs, separately, for critical probe- and cue-lure trials (Braver et al., 2009; Chiew \& Braver, 2014). The index was calculated as [(cue-lure) - (probelure) ] / (cue-lure) + (probe-lure)]. A correction was applied for trials in which participants made zero errors for both cue- and probe-lure trials: (error +0.5$)$ / (frequency of trials +1 ) as used in Braver et al. (2009) and Chiew and Braver (2014). Index values range from -1 to 1 and provide a composite measure of the relative engagement in proactive control, with higher values indicative of greater proactive control.

\footnotetext{
${ }^{5} \mathrm{RT}$ analyses with the $\mathrm{z}$-transformation revealed similar patterns as analyses without the transformation (Appendix $\mathrm{XI}$ ). The analyses with the $z$-transformation provide a clearer picture of age differences that controls for overall slower responses in older adults.
} 
Outliers. Participants with task error rates that were greater than 3 times the interquartile range were identified with box-and-whisker plots. Upon closer inspection, the high error rates were often due to poor performance in only one of the two blocks. Analyses were conducted with and without these outlier blocks and the pattern of results did not change. Therefore, these outlier blocks were included in the final reported analyses for greater statistical power.

Individual differences measures. Independent samples $t$-tests were conducted to examine for group differences in the individual differences measures. If differences were found, (e.g., age difference) on a particular measure, a correlation analysis was conducted to examine its relationship with a proactive index (as a marker of AX-CPT performance), but only if the index also showed the same group difference. If the correlation was significant, the measure was entered as a covariate and an ANCOVA on the proactive index was conducted. Correlations for Experiments 1 and 2 (combined) were also conducted to examine the relationship between AXCPT performance measures and cognitive tests (Appendix X).

Supplementary analyses. Supplementary analyses were conducted to compare performance across experiments. These analyses focused on the mood ratings and AX-CPT performance. The first set of analyses compared Experiments $1 \mathrm{~A}$ and $1 \mathrm{~B}$ to examine whether AX-CPT performance differed as a function of neutral versus negative induction, respectively. The second set of analyses compared Experiments 1A and 2A, which both consisted of neutral inductions but differed on the AX-CPT version used (letter versus face-letter, respectively). The next set compared Experiments 2A and 2B, which both used the face-letter AX-CPT but under differently valenced mood inductions. The last analysis conducted compared Experiments 1B and 2B as they both consisted of negative inductions but used different AX-CPT versions (letter versus face-letter, respectively). 


\section{Chapter 4: Experiments 1A and 1B}

\section{Age Differences in Letter AX-CPT Task under Neutral and Negative Mood}

The literature review revealed that younger adults typically exhibit proactive control whereas older adults exhibit reactive control (Braver et al., 2001, 2005). The overall objective of Experiment 1 was to examine the effect of neutral mood (Experiment 1A) and negative mood (Experiment 1B) inductions on proactive and reactive control in younger and older adults using the standard letter AX-CPT task.

\section{Experiment 1A: Age Differences in Letter AX-CPT Task under Neutral Mood}

Objectives and rationale. Experiment $1 \mathrm{~A}$ aimed to replicate typical age-related patterns in control using the standard letter AX-CPT task (with letter cues and probes). As a neutral induction procedure was also used in Experiment 2A, an additional goal was to check that the induction does not change typical age patterns. Having a neutral induction condition allowed for a neutral baseline condition to compare with the other studies of the dissertation (i.e. comparisons to Experiments 1B and 2A). Thus, younger and older participants also received neutral mood inductions prior to the AX-CPT task.

Hypotheses. It was hypothesized that neutral inductions would not change the typical age-related patterns of proactive and reactive control found using the standard letter AX-CPT task (Braver et al., 2001, 2005), as described below in H1 and H2.

$\boldsymbol{H}$ 1: Predominant proactive control in younger adults, and reduced proactive control in older adults. The predominant proactive control is reflected as poorer performance on cue- than probe-lure trials in younger adults only. Reduced proactive control in older adults is indexed as better performance (fewer errors, faster responses) on cue-lure trials and/or reduced proactive control indices in older than younger adults. 
$\boldsymbol{H}$ 2: Evidence of a shift to reactive control in older adults - indexed as poorer performance on probe-lure trials in older than younger adults, and similarly reflected as poorer performance on probe- than cue-lure trials within older adults.

\section{Experiment 1B: Age Differences in Letter AX-CPT Task under Negative Mood}

Objectives and rationale. The literature review identified studies that examined the effects of induced positive and neutral moods on proactive and reactive control in younger adults (Chiew \& Braver, 2014; van Wouwe, Band, \& Ridderinkhof, 2011). Only the effects of negative stimuli presented prior to each trial (rather than negative mood induced prior to the task) have been studied (Dreisbach, 2006; Fröber \& Dreisbach, 2012). Thus, no study has examined the effect of induced negative mood on proactive and reactive control in younger or older adults. Thus, Experiment 1B aimed to address this question by inducing younger and older adults into negative moods prior to the letter AX-CPT task. It is important to examine this question as it is possible that negative mood may have differential influences on older than younger adults, considering age differences in the effects of negative mood on components of cognitive control (e.g., working memory; Scheibe \& Blanchard-Fields, 2009; task-switching; Chu, 2014). Additionally, although it has been found that cognitive control in younger adults does not appear to be affected by trial-by-trial negative stimuli, it is unknown whether they would be affected when negative moods are intentionally induced instead. It is possible that previous studies with younger adults did not find an effect of negative emotion due to this distinction. Finally, results of Experiment 1B were also analyzed with those of Experiment $1 \mathrm{~A}$ (neutral induction) to examine whether control patterns in the letter AX-CPT differed under negative relative to neutral mood inductions. 
Hypotheses. As no studies have examined the effect of induced mood on proactive and reactive control, it was an open question as to what patterns would emerge. Several competing hypotheses were proposed, as described below.

$\boldsymbol{H}$ 3: Under induced negative mood, the same age-related patterns of cognitive control would emerge - see $\mathrm{H} 1$ and $\mathrm{H} 2$ (Experiment 1A). This hypothesis was based on the findings that negative pictures presented prior to each trial did not have an effect on younger adults' cognitive control (Dreisbach, 2006; Fröber \& Dreisbach, 2012). Thus, it was expected that negative mood induced by negative stimuli may not have an effect on older adults either.

H 4: Cross-experiment analyses with Experiment 1A would confirm H3 - there would be no differences in age-related patterns of cognitive control under induced negative or neutral moods.

$\boldsymbol{H}$ 5: Age differences in proactive control may be reduced or exaggerated - this was an alternative hypothesis to $\mathrm{H} 3$ and $\mathrm{H} 4$. Based on studies finding age differences in the effect of negative mood on components of cognitive control (e.g., Chu, 2014; Phillips, Smith, \& Gilhooly, 2002), the effect of induced mood might differ in younger and older adults. However, there were no hypotheses for the directionality of the effect, as studies found mixed effects of negative mood (e.g., facilitated task-switching; Chu, 2014; impaired planning; Phillips et al., 2002). 


\section{Experiment 1A: Age Differences in Letter AX-CPT Task under Neutral Mood}

Participants in Experiment 1A received neutral mood inductions prior to the standard letter AX-CPT task with letter cue and probe stimuli.

\section{Method}

\section{Participants}

Participants were 36 healthy younger (ages 18-26) and 31 healthy older adults (ages 6584). Ten younger adults were excluded due to high DASS scores that were in the range classified as having “extremely severe” depression and anxiety symptoms. Three older adults were excluded for different reasons: technical malfunction, discontinuation of the experiment due to illness, and misunderstanding the AX-CPT task instructions. The final sample included in the analyses consisted of 26 younger and 28 older adults. Demographic characteristics and cognitive performance of the participants are displayed in Table 2. 
Table 2

Participant Demographic Characteristics and Cognitive Performance

\begin{tabular}{|c|c|c|c|c|c|c|}
\hline \multirow[t]{2}{*}{ Characteristic } & \multicolumn{2}{|c|}{$\begin{array}{l}\text { Younger Adults } \\
(n=26)\end{array}$} & \multicolumn{2}{|c|}{$\begin{array}{l}\text { Older Adults } \\
(n=28)\end{array}$} & \multirow[t]{2}{*}{$p$-value } & \multirow[t]{2}{*}{$d$} \\
\hline & $M$ & $(S D)$ & $M$ & $(S D)$ & & \\
\hline Age & 19.12 & (2.03) & 71.21 & $(4.90)$ & $<.001^{* *}$ & 13.89 \\
\hline Female/male (ratio) & $22 / 4$ & & $16 / 12$ & & $.027^{x}$ & \\
\hline Years of education & 12.85 & (1.99) & 15.63 & (3.11) & $<.001^{* *}$ & 1.06 \\
\hline Health rating & 7.90 & $(0.80)$ & 8.21 & $(1.37)$ & .319 & 0.28 \\
\hline FTP & 54.58 & $(6.29)$ & 39.54 & $(10.86)$ & $<.001^{* *}$ & 1.69 \\
\hline PANAS-Positive & 25.15 & $(8.18)$ & 33.93 & $(6.72)$ & $<.001^{* *}$ & 1.17 \\
\hline PANAS-Negative & 16.08 & $(4.20)$ & 11.89 & (1.99) & $<.001^{* *}$ & 1.27 \\
\hline DASS-Depression & 6.92 & $(5.07)$ & 3.86 & $(4.77)$ & $.026 *$ & 0.62 \\
\hline DASS-Anxiety & 7.69 & $(5.71)$ & 2.50 & (3.38) & $<.001 * *$ & 1.11 \\
\hline Corsi & 0.64 & $(0.15)$ & $0.34^{\mathrm{a}}$ & $(0.18)$ & $<.001^{* *}$ & 1.81 \\
\hline Pattern comparison & 74.73 & $(10.74)$ & $49.31^{\mathrm{b}}$ & $(8.84)$ & $<.001 * *$ & 2.58 \\
\hline DCCST & 9.18 & $(0.59)$ & $7.57^{\mathrm{b}}$ & (1.18) & $<.001 * *$ & 1.73 \\
\hline Flanker & 9.62 & $(0.38)$ & $8.45^{b}$ & $(0.66)$ & $<.001^{* *}$ & 2.17 \\
\hline Vocabulary & 1539.88 & (111.01) & $2047.00^{b}$ & (576.31) & $<.001^{* *}$ & 1.22 \\
\hline SBT & & & 0.79 & $(1.00)$ & & \\
\hline
\end{tabular}

Note . Education = average number of years of formal education; health rating = report of self-rated health on a scale from 1 (poor) to 10 (excellent); FTP = Future Time Perspective Scale; PANAS-Positive/Negative = Positive Affect Negative Affect Schedule; DASS-Depression/Anxiety = Depression, Anxiety, and Stress Scales; Corsi = Corsi Block; Pattern comparison $=$ Pattern Comparison Processing Speed Test; DCCST $=$ Dimensional Change Card Sort Test; Flanker $=$ Flanker Inhibitory Control and Attention Test; Vocabulary = Picture Vocabulary Test; SBT = Short Blessed Test

${ }^{*} p<.05 .{ }^{* *} p<.001 .{ }^{x}$ denotes the use of chi-square statistic; all other analyses used independent $t$-tests

$d=$ Cohen's $d$ effect size calculation for between subjects

a one older adult did not complete the Corsi task due to a technical issue

${ }^{\mathrm{b}}$ two older adults did not complete any of the NIH Toolbox tasks due to technical issues 


\section{Letter AX-CPT Task}

The standard letter AX-CPT was used in this experiment and consisted of letters as cue and probe stimuli.

Stimuli. The valid cue and valid probe were always the letters A and X, respectively. Invalid cues and probes were all other letters of the alphabet except for $\mathrm{K}, \mathrm{V}$, and $\mathrm{Y}$ due to visual similarity to the letter $\mathrm{X}$ ( $n=21$ letters). All letter stimuli were presented in uppercase, bolded white Helvetica font, size 36-point, and were presented centrally against a black background.

Trial procedure. Each trial consisted of a cue-probe pair. Participants first saw a cue for $750 \mathrm{~ms}$, followed by an unfilled delay for $5000 \mathrm{~ms}$. A probe then appeared for $750 \mathrm{~ms}$. Participants had a response interval of $1750 \mathrm{~ms}$ from probe onset to respond. The inter-trial interval (ITI) was a blank screen lasting 1000 ms. All stimuli were presented continuously, one at a time. Responses were required for each cue ("non-target”) and probe ("target” or "nontarget”). All responses were collected using a keyboard. Target responses were made by pressing the "Z" key with the left index finger and non-target responses with the " $\mathrm{M}$ " key with the right index finger; keys were labelled “TARGET” or “NON-TARGET”, respectively. A target trial procedure is depicted in Figure 3.

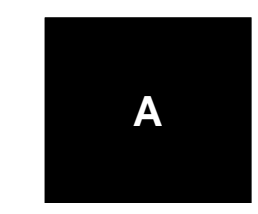

Valid Cue

$750 \mathrm{~ms}$

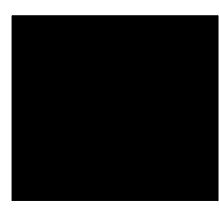

Delay

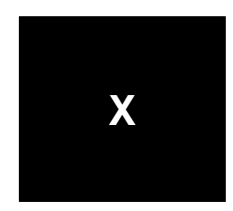

Valid Probe

$750 \mathrm{~ms}$

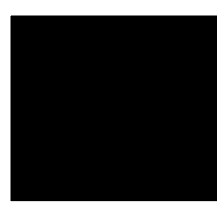

Response interval $5000 \mathrm{~ms}$ $1000 \mathrm{~ms}$

Time

Figure 3. Example of a target trial: letter A (valid cue) followed by letter X (valid probe). 
Trial types. Target trials were those in which the valid-cue A preceded the valid-probe X. Non-target trials consisted of cue-lure, probe-lure, and control trials. Cue-lure trials were those in which the valid-cue A preceded an invalid-probe. Probe-lure trials consisted of an invalid-cue followed by the valid-probe $\mathrm{X}$. Control trials were invalid-cue and invalid-probe pairings.

Following previous work (e.g., Braver et al., 2001, 2009), the distribution of trial types was as follows: the majority of trials in each block were target trials (70\%) and the rest were non-target trials (10\% of each non-target trial type). The high proportion of target trials was intended to elicit a strong, reliable bias towards target responses and the low proportion of nontarget trials was intended to increase the likelihood of interference on these trials (e.g., Braver et al., 2001, 2009). The order of trials was pseudo-randomly presented such that each block did not start or end with a non-target trial and no more than two non-target trials occurred in a row.

Task procedure. Participants began by reading the task instructions with the experimenter, which outlined that the target pair was the letter A followed by the letter X. They then completed a practice block (5 trials: 1 trial per non-target trial type and 2 target trials) and received accuracy feedback. Participants received feedback for the practice trials only; there was no feedback for the experimental trials. They also had the option to repeat the practice blocks until they understood the task. Participants completed two experimental blocks, each consisting of 100 trials: 70 target and 30 non-target trials (10 of each of the non-target trial types). Each block lasted approximately 14 minutes.

\section{Mood Ratings and Induction}

Participants received the neutral mood induction and mood ratings were assessed using the Self-Assessment Manikin, as described in the General Method section. 


\section{Procedure}

After arrival at the laboratory, participants received informed consent prior to beginning the experiment. Participants then tried on the headphones used for the mood induction to ensure fit and adequate volume. The volume was adjusted according to participant preference; participants heard a single tone at the laptop's volume level 40 and they indicated whether a louder or quieter level was preferred. Participants then began the experiment. First, the experiment reviewed the AX-CPT instructions with the participants. Participants then completed practice trials. Afterwards, the first mood rating was collected (pre-induction 1), which was immediately followed by the neutral mood induction. Immediately after the induction, participants rated their mood again (post-induction 1). They then began the first AX-CPT experimental block. Upon completion of the first block, participants rated their moods again (post-block 1). The same procedure was followed for the second half of the task which included the second neutral induction, followed by the second AX-CPT experimental block and the last three mood ratings (pre-induction 2, post-induction 2, and post-block 2). Upon completion of the AX-CPT task, participants responded to the mood induction questionnaire. After this point, participants had the option of taking a break. Following this, they completed a series of questionnaires and tests. The first was the Future Time Perspective Scale (FTP; Carstensen \& Lang, 1995). This was followed by the Positive and Negative Affect Schedule (PANAS; Watson et al., 1988) and the 21-item Depression Anxiety Stress Scales (DASS; Lovibond \& Lovibond, 1995). Participants then completed the NIH Toolbox tasks for approximately 20 minutes total. Finally, older adults were assessed for potential cognitive impairment using the Short Blessed Test (SBT; Katzman et al., 1983). Next, demographic and health information were collected on a background questionnaire. Upon completion, participants viewed a brief comedic video clip 
from Just for Laughs Gags as a mood reinstatement. All participants received a debriefing and were then granted course credit (younger adults) or received the cash incentive (older adults). In total, the experiment lasted approximately 1.5 to 2 hours.

\section{Results}

\section{Mood Ratings and Induction Questionnaire}

To examine the effect of the neutral mood induction on mood ratings, the SAM ratings for valence and arousal were submitted to two separate 2 (Age: younger vs. older) $\times 6$ (Time point) mixed ANOVAs.

Valence. For valence ratings, there was a significant main effect of time point, $F(3.63$, 188.78) $=11.20, p<.001, \eta_{\mathrm{p}}{ }^{2}=.18$. The main effect of age was trending significant, $p=.078$, with lower valence ratings for younger than older adults. These effects were qualified by an age by time point interaction, $F(5,260)=5.28, p<.001, \eta_{\mathrm{p}}{ }^{2}=.09$. Follow-up repeated measures ANOVAs with rating time point as a within-subjects factor for each age group revealed that time point was significant in younger, $F(3.39,84.733)=15.88, p<.000, \eta_{\mathrm{p}}{ }^{2}=.39$, but not older adults, $F(3.04,82.08)=2.07, p=.110, \eta_{\mathrm{p}}{ }^{2}=.07$. For younger adults, valence ratings were lower than baseline at each subsequent time point, $p s<.001$, except after the first induction, $p=.559$. There were no further changes in ratings after the first block, $p \mathrm{~s}>.306$. Additionally, younger and older adults differed only at the final two time points, $p s<.007$, and these remained significant after Bonferroni corrections. Specifically, younger adults $(M=5.27, S D=1.28)$ reported lower valence ratings than older adults $(M=6.39, S D=1.47)$ after the second induction, $p=.004$, and after the second AX-CPT block (younger: $M=5.08, S D=1.32$; older: $M$ $=6.21, S D=1.62), p=.007$. There were no age differences at baseline or at any of the other time points, $p s>.081$. Thus, younger adults' valence ratings declined after the first AX-CPT block 
before remaining stable, whereas older adults' ratings did not change from baseline. Despite these differences, the overall averages were within the middle range (4-6) that corresponds to neutral moods on the 9-point SAM rating scale, suggesting that the neutral induction was effective at achieving relatively neutral moods. Figure 4 (left panel) presents the mean valence ratings across time point.

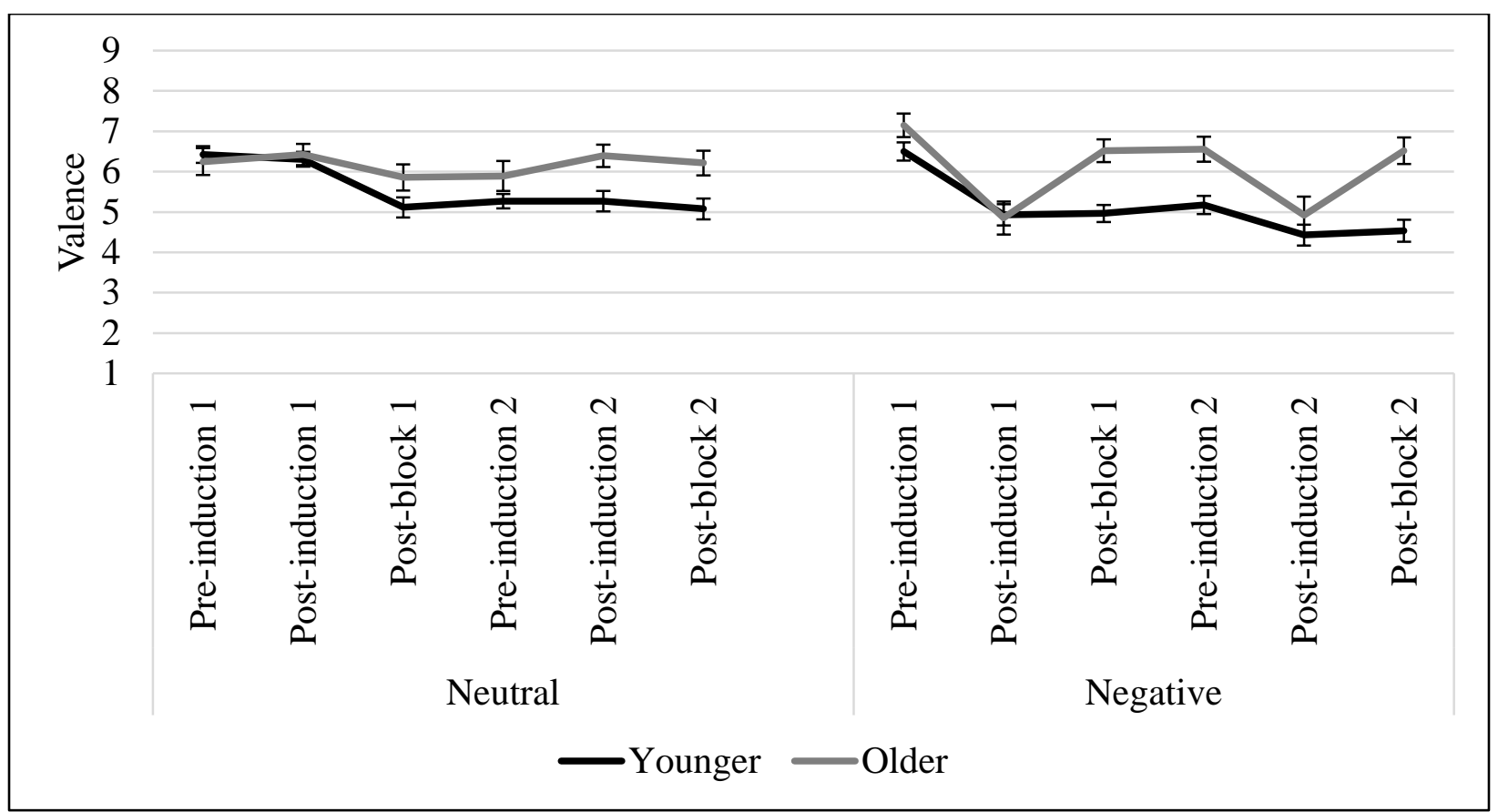

Figure 4. Mean valence ratings of mood across time point; Left panel: neutral mood induction (Experiment 1A); right panel: negative mood induction (Experiment 1B). Error bars denote standard error.

Arousal. The same mixed ANOVA was conducted on arousal ratings. There was a main effect of age, $F(1,52)=5.65, p=.021, \eta_{\mathrm{p}}{ }^{2}=.10$, with younger adults reporting lower arousal levels than older adults ${ }^{6}$. All other effects were not significant, $p s>.132$. The lack of time point

\footnotetext{
${ }^{6}$ Overall arousal rating (collapsed across time point) did not correlate with the proactive control index in RTs (which also showed significant age difference).
} 
effects or interactions suggest that arousal levels remained stable across the various time points for both groups. Figure 5 (left panel) presents the mean arousal ratings across time point.

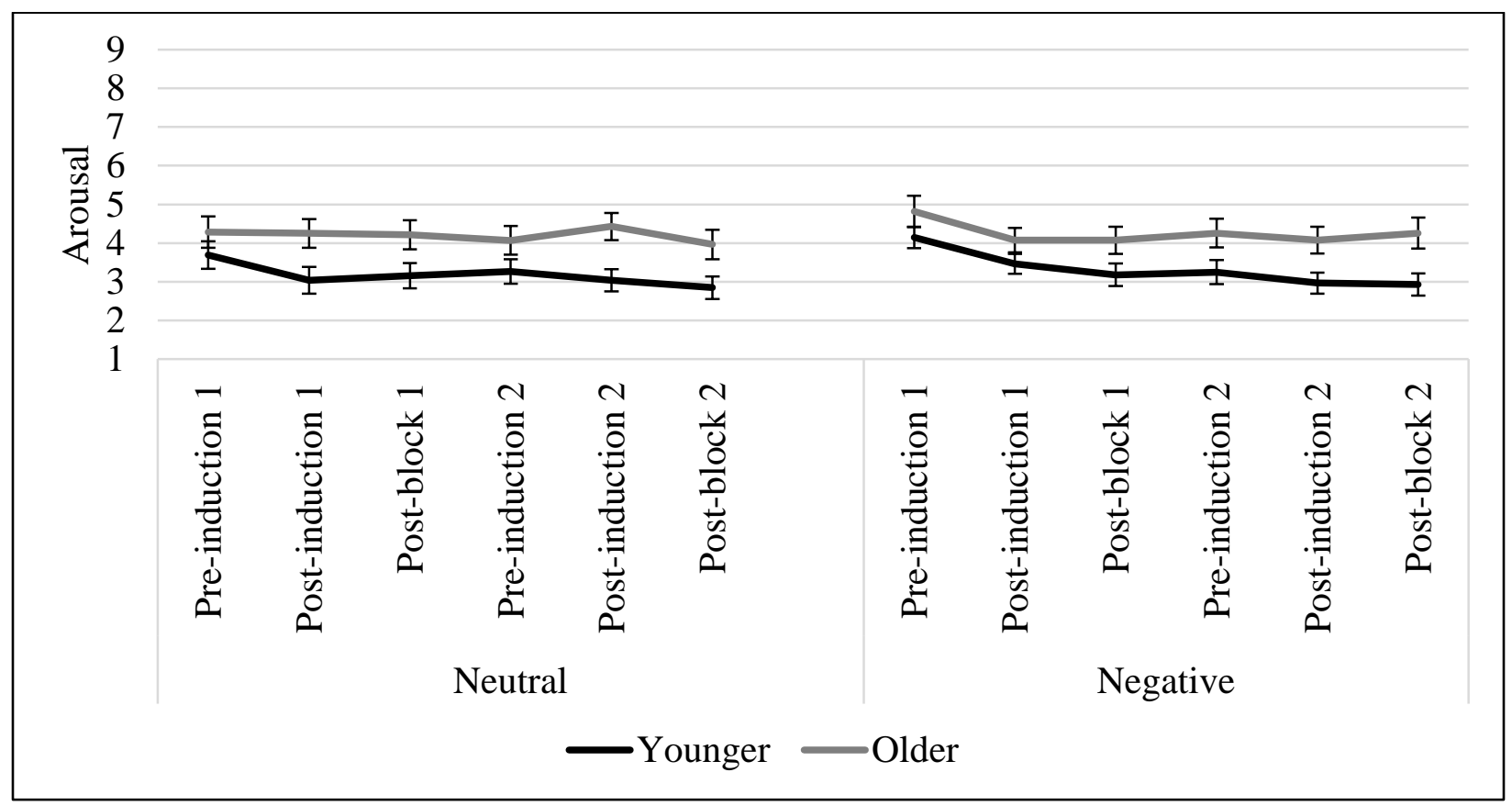

Figure 5. Mean arousal ratings of mood across time point; Left panel: neutral mood induction (Experiment 1A); right panel: negative mood induction (Experiment 1B). Error bars denote standard error.

Induction questionnaire. Analyses were conducted to examine age differences on responses to the induction questionnaire. Results of $t$-tests revealed no age differences for how familiar participants were with the sounds heard during the induction, $p=.563$, and both groups reported similar ratings of induction effectiveness, $p=.582$. For the question on slideshow processing, $74.07 \%$ indicated that they were paying attention, $14.81 \%$ indicated they were trying to memorize, and $11.11 \%$ indicated they were doing neither. To examine age differences in these responses, chi-square analyses were conducted for each category of processing (e.g., attention). 
These analyses revealed no age differences for each category, $\chi^{2} s<2.09$, $p s>.09$. No further analyses were conducted with these data as there were no age differences.

\section{Letter AX-CPT Performance}

The results for target and non-target trials were analyzed separately, for both error rates and z-transformed median response times (RTs), as presented in the left panel of Figure 6 (error rates) and Figure 7 (RTs).

Two older adults had very high error rates in only one of the two blocks ( $M s>0.67)$. For one of these participants, the error rate was high in the first block $(M=0.79)$ due to incorrect responses for target trials. It appears this participant pressed the wrong key ( $M$ instead of Z key) to indicate a correct target response but was corrected in the second block ( $M=0$ across all trials). The other participant had a high error rate in the second block $(M=0.67)$ due to lack of responses across all trial types. The error rate in the first block for this participant was low $(M=$ 0.40), suggesting that the lack of responses in the second block may have been caused from a keyboard input issue. Omitting these high error blocks for both participants did not change the pattern of results. Thus, the reported results include these blocks.

Only response time data for correct responses were used (91.89\% of all trials). One younger adult did not have any correct responses for probe-lure trials and as such, no correct response time data for that trial type (i.e., missing).

Target trials.

Error rates. Independent samples $t$-test comparing younger and older adults revealed no age differences, $t(52)=0.77, p=.445$. 


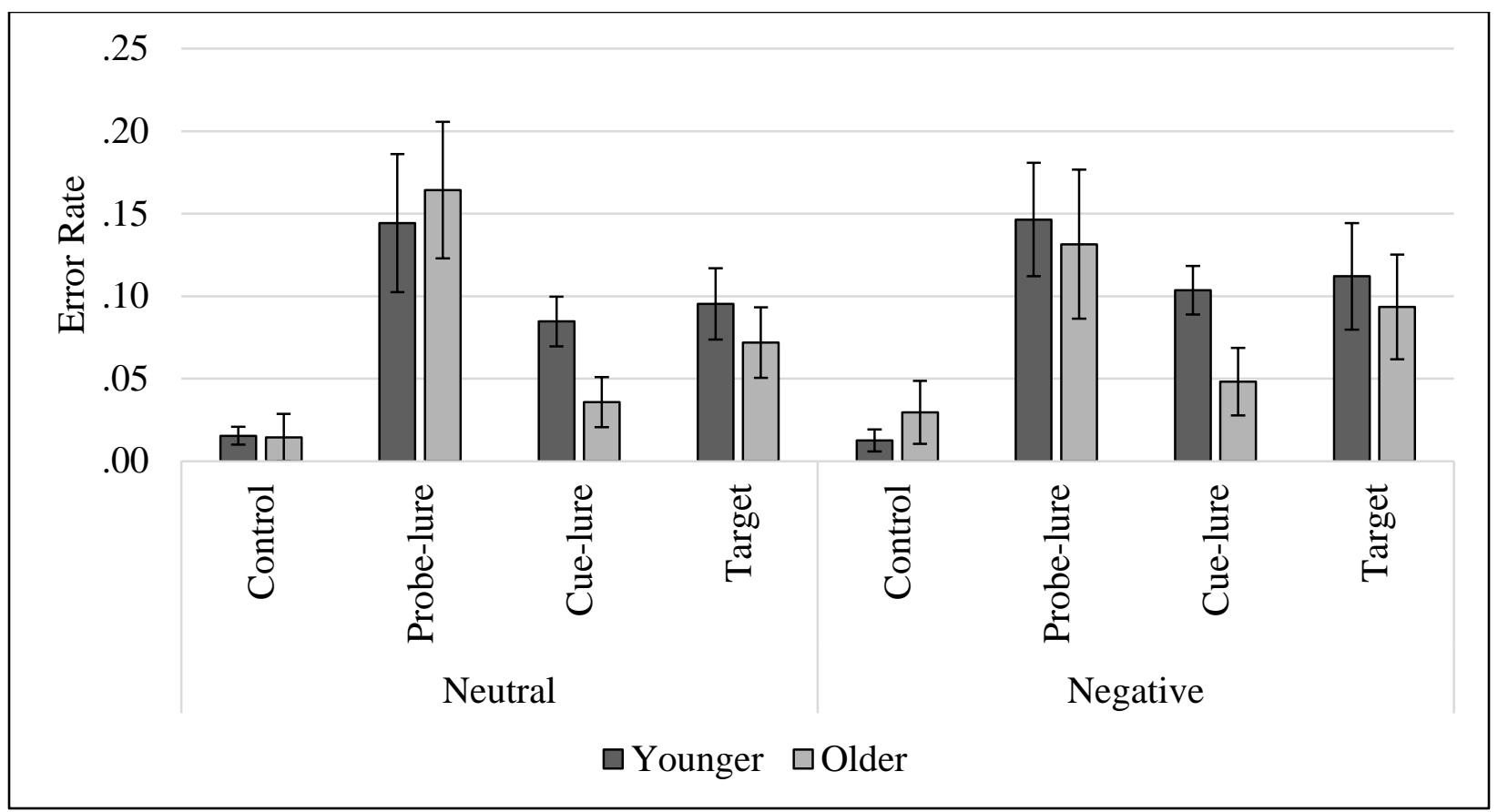

Figure 6. Mean error rates by trial type; Left panel: neutral mood induction (Experiment 1A), right panel: negative mood induction (Experiment 1B). Error bars denote standard error.

Response times. Independent samples $t$-test comparing younger and older adults revealed age differences, $t(52)=2.11, p=.040$. Younger adults $(M=-0.33, S D=0.11)$ had slightly longer RTs relative to older adults $(M=-0.40, S D=0.12)$. 


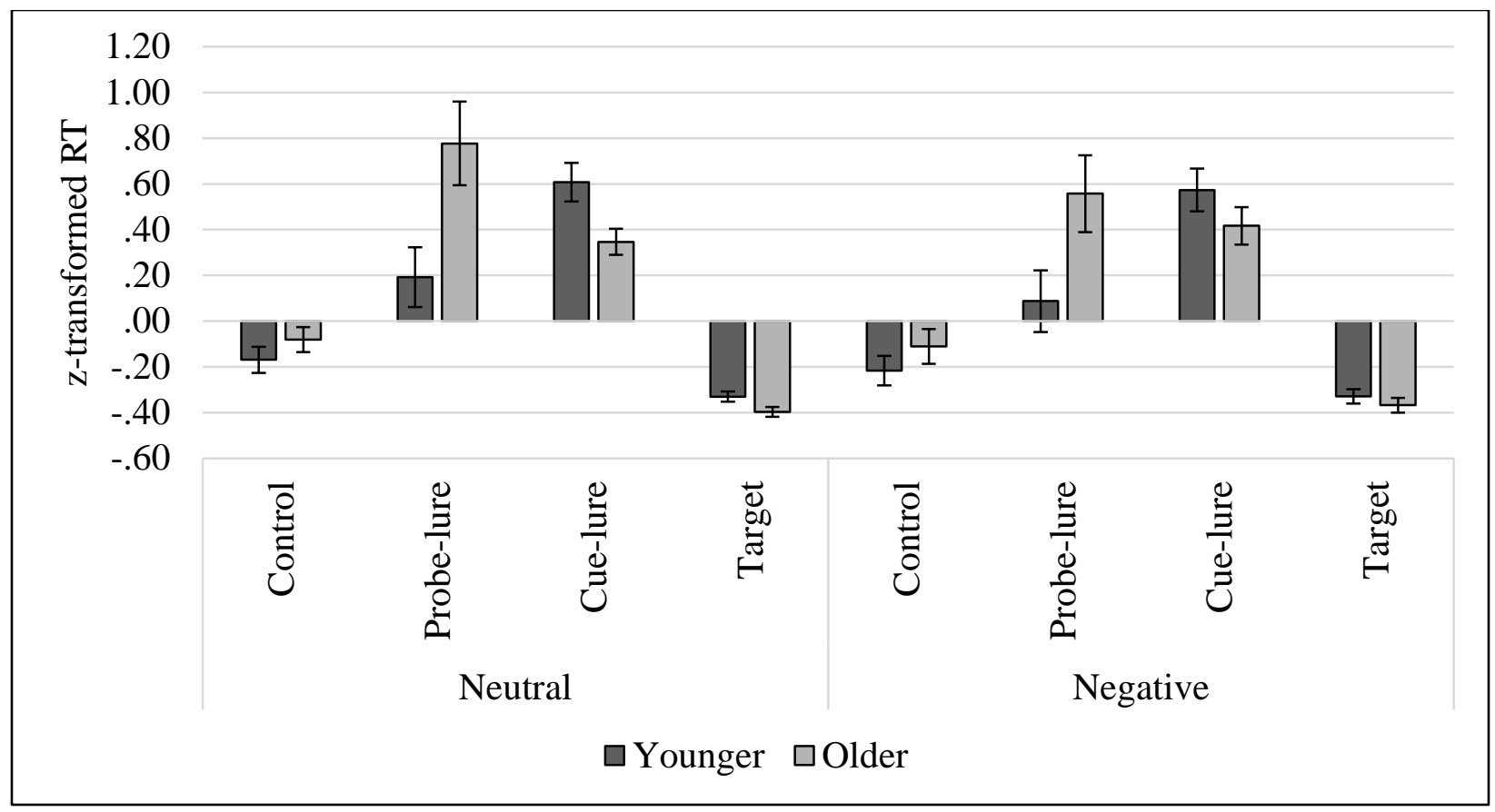

Figure 7. Mean z-transformed RTs by trial type; Left panel: neutral mood induction (Experiment 1A), right panel: negative mood induction (Experiment 1B). Error bars denote standard error.

\section{Non-target trials.}

Error rates. The 2 (Age: younger, older) $\times 3$ (Trial type: control, probe-lure, cue-lure) mixed ANOVA revealed a main effect of trial type, $F(1.11,57.59)=15.63, p<.001, \eta_{p}{ }^{2}=.23$. Planned contrasts revealed higher error rates for both probe-lure $(M=0.15, S D=0.21)$ and cuelure $(M=0.06, S D=0.08)$ than control $(M=0.01, S D=0.06)$ trials, $p s<.001$. Error rates were higher for probe-lure than cue-lure trials, $p=.004$. There was no main effect of age or an age by trial type interaction, $p s>.385$. Nonetheless, planned contrasts were conducted to examine hypothesized age differences for cue- and probe-lure trials. Independent samples $t$-tests revealed age differences for cue-lure trials only, with higher error rates in younger $(M=0.08, S D=0.08)$ than older $(M=0.04, S D=0.08)$ adults, $p=.027$. Separate paired samples $t$-tests for each age group were also conducted to examine hypothesized age differences for cue- versus probe-lure 
trials. For younger adults, error rates were similar in cue-lure $(M=0.08, S D=0.08)$ and probelure $(M=0.14, S D=0.21)$ trials, $p=.229$. However, error rates were higher for probe-lure $(M=$ $0.16, S D=0.22)$ than for cue-lure $(M=0.04, S D=0.08)$ trials in older adults only, $p=.005$. These differences are consistent with prior studies of age-related differences in proactive versus reactive control. There was some evidence of greater proactive control in younger relative to older adults (indexed by more errors on cue-lure trials in younger than older adults) and greater reactive than proactive control in older adults (indexed by increased errors on probe- versus cuelure trials). However, the age by trial type interaction did not reach significance and thus these results should be interpreted with caution (Figure 6, left panel).

Response times. Similar to the error rate analyses, there was a main effect of trial type, $F(1.25,63.60)=20.84, p<.001, \eta_{\mathrm{p}}{ }^{2}=.29$, with faster responses to control trials $(M=-0.12, S D$ $=0.29)$ relative to probe- $(M=0.50, S D=0.88)$ and cue-lure $(M=0.47, S D=0.39)$ trials. The planned contrast comparing probe- vs. cue-lure trials was not significant, $p=.885$. The main effect of trial type was qualified by an age by trial type interaction, $F(2,102)=7.76, p=.001$, $\eta_{\mathrm{p}}{ }^{2}=.13$. Independent samples $t$-tests revealed that the two age groups performed similarly for control trials, $p=.265$, but differently for interference trials. Relative to younger adults, older adults responded slower to probe-lure trials, $p=.014$, but faster to cue-lure trials, $p=.012$. Additionally, planned paired samples $t$-tests showed that both age groups responded faster to control relative to interference trials, $p \mathrm{~s}<.014$. However, there were age differences for the interference trials. Younger adults responded slower to cue- $(M=0.61, S D=0.43)$ vs. probe-lure $(M=0.19, S D=0.13)$ trials, $p=.026$, whereas older adults exhibited the opposite pattern, with slower responses to probe- $(M=0.78, S D=0.97)$ vs. cue-lure trials $(M=0.35, S D=0.30), p=$ 0.054. The main effect of age was not significant, $p=.112$. These results clearly show evidence 
of reduced proactive control and greater reactive control in older relative to younger adults (Figure 7, left panel).

Proactive index. Analyses were conducted on the proactive indices for errors and RTs (see General Methods: Data Analysis section for index formulae) to examine differences between age groups. First, one-sample $t$-tests were conducted for each age group to test whether index values were significantly different than zero. These tests revealed a proactive index that was greater than zero for RTs for younger adults $(M=0.05, S D=0.11), t(24)=2.33, p=.028$. However, the proactive index for errors $(M=-0.02 . S D=0.69)$ did not differ from zero, $p=$ .885. For older adults, the proactive index for both errors $(M=-0.28, S D=0.80)$ and RTs $(M=$ $0.05, S D=0.14)$ were not significantly different than zero, $p s>.088$.

To compare index values in younger versus older adults, separate univariate ANOVAs were conducted. For errors, there was no difference in the proactive index for younger and older adults, $p=.217$ (Figure 8, left panel). 


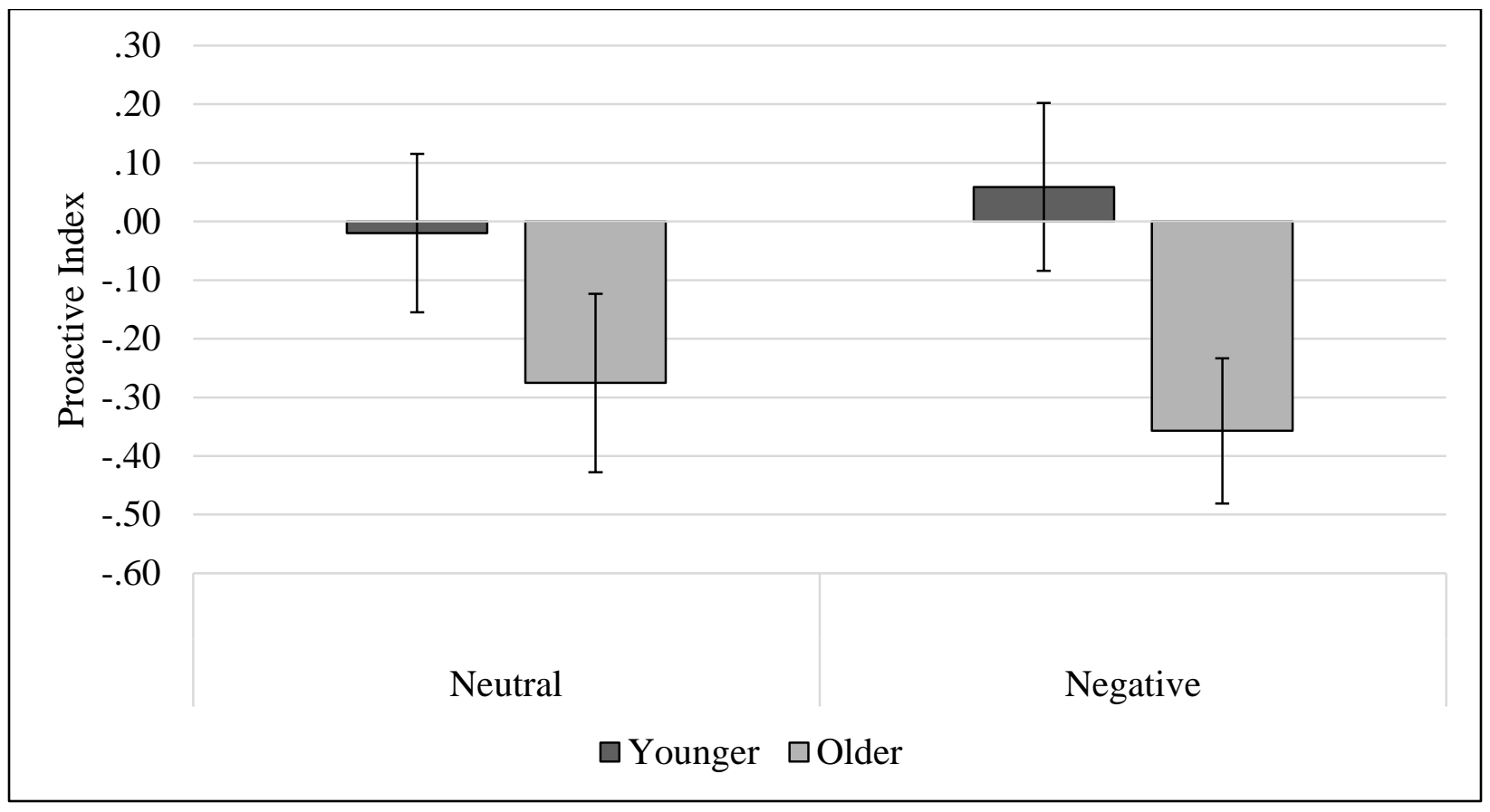

Figure 8. Mean proactive index for errors; Left panel: neutral mood induction (Experiment 1A), right panel: negative mood induction (Experiment 1B). Error bars denote standard error.

For RTs, the proactive index was larger for younger $(M=0.05, S D=0.11)$ relative to older adults $(M=-0.05, S D=0.14), F(1,52)=7.97, p=.007, \eta_{\mathrm{p}}{ }^{2}=.135$. These results reflect the more evident age differences in proactive control in the RT compared to the error data (Figure 9, left panel). 




Figure 9. Mean proactive index for median RTs; Left panel: neutral mood induction (Experiment 1A), right panel: negative mood induction (Experiment 1B). Error bars denote standard error.

\section{Individual Differences Measures}

There were significant age differences on most demographic characteristics, questionnaires, and cognitive tests. The correlation analyses suggested that some of these variables showed significant correlations with the RT proactive index, which also showed a significant age difference (larger proactive index for younger than older adults): PANASPositive, $r(53)=-.30, p=.029$; Corsi block, $r(52)=.39, p=.004$; and DCCST, $r(51)=.41, p=$ .003. To examine whether these variables may contribute to the age difference found in the proactive index, an ANCOVA was conducted with age as a between-subjects factor and with all three variables entered as covariates. The results suggested that the age difference in the RT proactive index was no longer significant, $p=.339$. Only the main effect of DCCST was significant, $F(1,49)=4.85, p=.033, \eta_{\mathrm{p}}^{2}=.097$, with higher scores for younger than older adults. All other effects were not significant, $F \mathrm{~s}<2.50$, $p \mathrm{~s}>.121$. This suggests that age-related 
differences in positive emotions (as measured on the PANAS), task-switching (DCCST), and working memory (Corsi block) may have been related to older adults’ reduced proactive control as measured in RTs. However, a direct contribution is unlikely; rather, the correlations of these variables with age (PANAS-Positive: $r(54)=0.51$; Corsi block, DCCST, proactive index RT, $r$ s $<-0.41$, ps $<.002)$ suggest that the covariance is likely commonly driven by age.

\section{Discussion}

The purpose of Experiment 1A was to replicate reported age-related patterns of reactive and proactive control in the letter AX-CPT and to pilot a neutral mood induction. Experiment $1 \mathrm{~A}$ also provides a standard comparison baseline for later experiments. Participants completed two blocks of the letter AX-CPT, with letters as probe and cue stimuli. They also received neutral mood inductions before starting each AX-CPT block. It was hypothesized (H 1, H 2) that the neutral induction would not change the typically reported age-related patterns of AX-CPT performance (e.g., Braver et al., 2001, 2005).

First, the neutral induction was relatively successful at keeping mean valence ratings in a neutral range, coupled with low arousal ratings. The induction appeared to slightly lower the mood of younger adults from a relatively more positive baseline to neutral levels. Older adults more consistently rated their moods as being somewhat positive across time. This pattern in older adults is not surprising considering the literature on age-related positivity bias (Carstensen et al., 2011). However, there were no age differences in the rating of induction effectiveness.

The data also replicated findings of an age-related shift in cognitive control patterns in the letter AX-CPT that is consistent with the literature. Specifically, studies have found that evidence of age-related reductions in proactive control most consistently manifests as better cue- 
lure performance in older than younger adults (Braver et al., 2001, 2005). In the current experiment, this age difference on cue-lure trials was seen in both the error and RT data.

Furthermore, the DMC predicts that older adults use reactive rather than proactive control to respond on the AX-CPT task. Evidence of reactive control in older adults is more consistently seen as disproportionately slowed RTs (after taking into account general age-related slowing) on probe-lure trials relative to younger adults ${ }^{7}$. However, a similar age difference is not always found in the error data (Braver et al., 2005; Braver, 2012; see Introduction of this dissertation for further details explaining this difference). This was also the case in the current experiment (i.e., more clear evidence of theoretically-predicted age by trial interactions in the RT than error data). The lack of this age difference in the error data appeared to be driven by similarly high rates of probe-lure errors in both age groups. For older adults, high rates of probe-lure errors appeared to be accompanied by slow probe-lure RTs, suggesting that their performance on these trials is not attributed to a speed-accuracy trade-off. However, a trade-off may be present for younger adults, as high probe-lure errors were accompanied by fairly fast probe-lure RTs. As the RT data represent only the speed of correct responses (and not of incorrect ones), it may be the case that while younger adults do occasionally make probe-lure errors (possibly due to attentional lapses), their responses tend to be fast when they are correct on these trials. Indeed, probe-lure RTs are almost as fast as those of control trials. This suggests that they are processing and maintaining the invalid cue and can prepare a non-target response accordingly to enable fast responding. This is consistent with the DMC account and predictions of proactive control in younger adults. In contrast, these patterns are not observed in older adults, who tend to be disproportionately slower

\footnotetext{
${ }^{7}$ Theoretically, reactive control can also be reflected as poorer probe- than cue-lure performance (e.g., Paxton et al., 2008), but studies are not as consistent in analyzing or reporting whether this difference was significant in their results (e.g., Braver et al., 2005; Paxton et al., 2006).
} 
on probe-lure trials (relative to the other trial types) even when they are correct, suggesting use of reactive control to guide correct responding instead. However, the mechanism underlying younger adults' tendency to make as many probe-lure errors as older adults is unclear and warrants further research for refinement of DMC predictions. Nevertheless, results of Experiment 1A replicated typical age-related control patterns under neutral induction and could serve as baseline comparisons for subsequent experiments of this dissertation. As such, Experiment 1B was conducted next to examine the effect of induced negative moods. 


\section{Experiment 1B: Age Differences in Letter AX-CPT Task under Negative Mood}

Participants in Experiment 1B received negative mood inductions prior to the standard letter AX-CPT task with letter cue and probe stimuli.

\section{Method}

\section{Participants}

Participants were 34 healthy younger (ages 18-28) and 32 healthy older adults (ages 6583). Two younger adults were excluded due to high DASS scores that were in the range classified as "extremely severe"; three were excluded due to current diagnoses of depression or anxiety disorders; and one was excluded due to prolonged unconsciousness. Two older adults were excluded as they discontinued the experiment; two were excluded due to high SBT scores; one was excluded due to misunderstanding of the AX-CPT task. The final sample included in the analyses consisted of 28 younger and 27 older adults (Table 3). 
Table 3

Participant Demographic Characteristics and Cognitive Performance

\begin{tabular}{|c|c|c|c|c|c|c|}
\hline \multirow[t]{2}{*}{ Characteristic } & \multicolumn{2}{|c|}{$\begin{array}{l}\text { Younger Adults } \\
(n=28)\end{array}$} & \multicolumn{2}{|c|}{$\begin{array}{l}\text { Older Adults } \\
(n=27)\end{array}$} & \multirow[t]{2}{*}{$p$-value } & \multirow[t]{2}{*}{$d$} \\
\hline & $M$ & $(S D)$ & $M$ & $(S D)$ & & \\
\hline Age & 20.32 & $(3.00)$ & 70.74 & $(5.22)$ & $<.001^{* *}$ & 11.84 \\
\hline Female/male (ratio) & $26 / 2$ & & $19 / 8$ & & $.031^{x}$ & \\
\hline Years of education & 14.11 & $(1.72)$ & 17.00 & $(4.24)$ & $.002 * *$ & 0.89 \\
\hline Health rating & 7.73 & $(1.14)$ & 8.13 & (1.67) & .307 & 0.28 \\
\hline FTP & 52.32 & $(7.66)$ & 43.19 & $(9.58)$ & $<.001^{* *}$ & 1.05 \\
\hline PANAS-Positive & 22.96 & $(4.91)$ & 36.48 & $(6.47)$ & $<.001^{* *}$ & 2.35 \\
\hline PANAS-Negative & 14.82 & $(4.29)$ & 12.93 & (3.69) & .085 & 0.47 \\
\hline DASS-Depression & 7.43 & (5.98) & 4.37 & $(4.00)$ & $.031 *$ & 0.60 \\
\hline DASS-Anxiety & 7.14 & (5.97) & 3.04 & $(3.70)$ & $.004^{*}$ & 0.83 \\
\hline Corsi & 0.64 & $(0.14)$ & 0.32 & $(0.19)$ & $<.001^{* *}$ & 1.92 \\
\hline Pattern comparison & 81.00 & $(8.52)$ & 52.04 & (11.53) & $<.001^{* *}$ & 2.86 \\
\hline DCCST & 9.29 & $(0.64)$ & 8.08 & $(0.98)$ & $<.001^{* *}$ & 0.69 \\
\hline Flanker & 9.54 & $(0.30)$ & 8.72 & $(0.64)$ & $<.001^{* *}$ & 1.64 \\
\hline Vocabulary & 1582.11 & (155.18) & 1965.71 & (220.86) & $<.001^{* *}$ & 2.01 \\
\hline SBT & & & 0.22 & $(0.16)$ & & \\
\hline
\end{tabular}

Note. Education = average number of years of formal education; health rating = report of self-rated health on a scale from 1 (poor) to 10 (excellent); FTP = Future Time Perspective Scale; PANAS-Positive/Negative = Positive Affect Negative Affect Schedule; DASS-Depression/Anxiety = Depression, Anxiety, and Stress Scales; Corsi = Corsi Block; Pattern comparison $=$ Pattern Comparison Processing Speed Test; DCCST $=$ Dimensional Change Card Sort Test; Flanker $=$ Flanker Inhibitory Control and Attention Test; Vocabulary = Picture Vocabulary Test; SBT = Short Blessed Test

${ }^{*} p<.05 .{ }^{* *} p<.001 .{ }^{x}$ denotes the use of chi-square statistic; all other analyses used independent $t$-tests $d=$ Cohen's d effect size calculation for between subjects 


\section{Letter AX-CPT Task}

The same standard letters AX-CPT was used as in Experiment 1A, consisting of letters as cue and probe stimuli.

\section{Mood Ratings and Induction}

The mood rating and induction procedures were the same as in Experiment $1 \mathrm{~A}$ except participants received the negative instead of the neutral mood induction.

\section{Procedure}

The procedure of the study was identical to Experiment $1 \mathrm{~A}$.

\section{Results}

\section{Mood Ratings and Induction Questionnaire}

Valence. The main effect of age was significant, $F(1,53)=9.24, p=.004, \eta_{\mathrm{p}}{ }^{2}=.15$, with younger adults reporting lower moods than older adults ${ }^{8}$. These effects were qualified by an age by time point interaction, $F(5,265)=6.71, p<.001, \eta_{\mathrm{p}}{ }^{2}=.11$. Separate repeated measures ANOVAs for each age group revealed main effects of time point for both age groups, $p s<.001$. Follow-up multiple comparisons were conducted. For younger adults, ratings were lower after the second than first induction and were higher at the second pre-induction compared to subsequent time points, $p \mathrm{~s}<.003$. For older adults, ratings did not differ between the first and second post-induction time points, $p=.795$. Both post-induction ratings were lower compared to the other time points, $p s<.001$; these time points did not differ from each other, $p s>.832$. The two age groups did not differ at baseline or immediately after the two inductions, $p s>.084$. For

\footnotetext{
${ }^{8}$ Overall valence rating (collapsed across time point) did not correlate with the proactive control index for errors or RTs (both of which also showed significant age differences).
} 
all other time points, younger adults reported lower valence ratings than older adults, $p s<.001$. Figure 4 (right panel) displays the valence ratings.

In summary, the negative mood induction was effective at lowering mood (relative to baseline) for both younger and older adults. The induction also resulted in equivalent mood ratings for younger and older adults at both post-induction time points. However, ratings after the first post-induction remained low in younger adults whereas older adults reported higher ratings upon completing each AX-CPT block and also before the second pre-induction time point.

Arousal. Arousal ratings were submitted to the same mixed ANOVA. There was a main effect of time point, $F(3.15,166.96)=6.71, p<.001, \eta_{\mathrm{p}}{ }^{2}=.11$. Planned simple contrasts revealed arousal ratings that were significantly lower relative to baseline, $p s<.006$. Multiple comparisons showed that arousal ratings for subsequent ratings did not differ from each other, $p$ s $>$.090. There was also a main effect of age, $F(1,53)=5.83, p=.019, \eta_{\mathrm{p}}{ }^{2}=.10$, with higher valence ratings in older than younger adults ${ }^{9}$. There was no age by time point interaction, $p=$ .413. The arousal ratings are presented in Figure 5 (right panel).

Induction questionnaire. Results revealed no age differences for how familiar participants were with the music heard during the induction, $p=.784$, familiarity with classical music, $p=.063$. However, younger adults $(M=4.93, S D=2.14)$ reported lower ratings of induction effectiveness than older adults $(M=6.48, S D=2.53), p=.017$. However, these ratings did not correlate with critical proactive indices for either RTs or errors, $p$ s $>$.133, so no further analyses were conducted using this variable. For the question on slideshow processing, 87.27\% indicated that they were paying attention, 3.64\% indicated they were trying to memorize, and

\footnotetext{
${ }^{9}$ Overall arousal rating (collapsed across time point) significantly correlated with the proactive control index for RTs, $r(55)=-.32, p=.018$. Entering overall arousal as a covariate in an ANCOVA with age as a between-subjects factor revealed that age was no longer significant in the proactive control index for RTs.
} 
9.09\% indicated they were doing neither. To examine age differences in these responses, chisquare analyses were conducted for each category of processing. These analyses revealed no age differences for each category, $\chi^{2} s<2.00, p s>.157$.

\section{AX-CPT Performance}

Three younger and three older adults had error rates that were very high $(M \mathrm{~s}>0.42)$ in only one of the two blocks, likely due to missing responses and key switches. Omitting these high error blocks did not change the pattern of results. Thus, the reported results include these blocks for greater statistical power. Only response times for correct responses were included in the final analysis (90.44\% of all trials).

\section{Target trials.}

Error rates. Independent samples t-test comparing younger and older adults on target trials revealed no age differences, $t(53)=0.41, p=.683$ (Figure 6 , right panel).

Response times. Independent samples $t$-test comparing younger and older adults on target trials also revealed no age differences, $t(53)=0.86, p=.393$ (Figure 7 , right panel).

\section{Non-target trials.}

Error rates. The 2 (Age: younger, older) $\times 3$ (Trial type: control, probe-lure, cue-lure) mixed ANOVA revealed a main effect of trial type, $F(1.17,61.92)=14.82, p<.001, \eta_{\mathrm{p}}{ }^{2}=.22$. Planned contrasts revealed higher error rates for both probe-lure $(M=0.14, S D=0.21)$ and cuelure $(M=0.08, S D=0.10)$ than control $(M=0.02, S D=0.07)$ trials, $p s<.001$. Error rates were higher for probe-lure than cue-lure trials, $p=.021$. There was no main effect of age or an age by trial type interaction, $p s>.249$. However, to examine age differences for cue- vs. probe-lure trials, independent samples $t$-tests were conducted. Age differences were found for cue-lure trials only, with higher error rates in younger $(M=0.10, S D=0.08)$ than older $(M=0.05, S D=0.11)$ 
adults, $p=.031$. Separate paired samples $t$-tests for each age group were also conducted to test for hypothesized age differences in cue- vs. probe-lure trials. For younger adults, error rates were similar in probe-lure $(M=0.15, S D=0.18)$ and cue-lure $(M=0.10, S D=0.08)$ trials, $p=.279$. However, older adults showed higher error rates for probe-lure $(M=0.13, S D=0.23)$ than cuelure $(M=0.05, S D=0.11)$ trials, $p=.029$ (Figure 6, right panel).

Overall, the accuracy rates replicate the same patterns found in Experiment 1A. Although the age by trial interaction did not reach significance, there was evidence of greater proactive control in younger relative to older adults (more errors on cue-lure trials in younger than older adults).

Response times. The same mixed ANOVA on RTs revealed a main effect of trial type, $F(1.25,66.17)=21.67, p<.001, \eta_{\mathrm{p}}{ }^{2}=.29$, with faster responses to control trials $(M=-0.16, S D$ $=0.37)$ relative to probe- $(M=0.32, S D=0.82)$ and cue-lure $(M=0.50, S D=0.47)$ trials. The planned contrast comparing probe- and cue-lure trials was not significant, $p=.217$. There was no main effect of age, $p=.157$, but there was a significant age by trial type interaction, $F(2,106)=$ 4.60, $p=.012, \eta_{\mathrm{p}}^{2}=.08$. Planned paired samples $t$-tests showed that both age groups responded faster on control relative to interference trials, $p s<.017$. However, the two age groups differed across the two types of interference trials. Younger adults had longer RTs on cue- ( $M=0.57, S D$ $=0.50)$ than on probe-lure $(M=0.09, S D=0.71)$ trials, $p=.013$, whereas no difference was found for probe- $(M=0.56, S D=0.87)$ compared to cue-lure $(M=0.42, S D=0.43)$ trials in older adults, $p=0.502$. Independent samples $t$-tests on probe-lure trials also revealed that younger adults $(M=0.09, S D=0.71)$ had reduced RTs relative to older adults $(M=0.56, S D=$ 0.87). However, there were no age differences for cue-lure or control trials, $p s>.217$. These results are similar to those found in Experiment 1A, with evidence of proactive control in 
younger adults (indexed by larger RTs on cue- than probe lure trials). Unlike in Experiment 1A, older adults did not exhibit evidence of a reactive control preference (Figure 7, right panel).

Proactive index. The proactive indices were calculated and analyzed in the same way as in Experiment 1A. The proactive index for errors was not significantly different from zero for younger adults $(M=0.06, S D=0.76), p=.684$, but was significantly lower than zero for older adults $(M=-0.36, S D=0.64), p=.008$ (Figure 8, right panel). The proactive index for RT was significantly greater than zero for younger adults $(M=0.06, S D=0.13), p=.014$, but was not significantly different from zero for older adults $(M=-0.02, S D=0.13), p=.517$. Univariate ANOVAs comparing age differences revealed that younger adults had higher proactive index values than older adults for both errors, $F(1,54)=4.79, p=.033, \eta_{\mathrm{p}}{ }^{2}=.083$, and RTs, $F(1,54)=$ 5.35, $p=.025, \eta_{\mathrm{p}}{ }^{2}=.092$. Again, these results largely mimic those found in Experiment $1 \mathrm{~A}$ (Figure 9, right panel).

\section{Individual Differences Measures}

Similar to Experiment 1A, there were significant age differences in most demographic characteristics, questionnaires, and cognitive tests. Some of these variables correlated with the error and RT proactive indices, which also showed significant age differences (larger proactive index for younger than older adults). Specifically, the proactive index in errors correlated with education, $r(55)=-.35, p=.010$, and DCCST, $r(52)=.30, p=.032$. The proactive index in RTs correlated with: education, $r(55)=-.37, p=.006$; FTP, $r(55)=.28, p=.041$; DCCST, $r(52)=$ $.33, p=.017$; and vocabulary, $r(51)=-.30, p=.030$. The ANCOVA on proactive index in errors with education and DCCST entered as covariates revealed that the age difference was no longer significant, $p=.711$. All other effects were also not significant, $F \mathrm{~s}<2.95, p s>$.092. The ANCOVA on proactive index in RTs with education, FTP, DCCST, and vocabulary entered as 
covariates also revealed that the age difference was no longer significant, $p=.933$, and all other effects were not significant, $F s<2.24, p s>.141$. Similar to Experiment $1 \mathrm{~A}$, these effects are likely to reflect the common effect of age.

\section{Cross-experiment (1A vs. 1B) Analyses}

To examine whether performance on the letter AX-CPT varied by neutral versus negative mood inductions, the results from Experiments 1A (neutral induction) and 1B (negative induction) were compared. First, mood ratings were compared across the two experiments to check whether mood ratings differed in the expected directions.

Mood ratings. Ratings for the two induction groups were compared at each time point, collapsed across age. As expected, valence ratings were lower in the negative (Ms $<4.89$, SDs $<$ 1.93) than neutral $(M s>5.85$, SDs $<1.48)$ induction groups at both post-induction time points, ps $<.001$. Ratings were trending significance at baseline, $t(107)=1.78, p=.078$, with slightly higher ratings in the negative $(M=6.82, S D=1.39)$ than neutral $(M=6.33, S D=1.45)$ group. However, ratings were similar in both induction groups at all other time points, $p s>.384$. The same analyses on arousal ratings revealed no significant induction group differences at any time point, $p s>.201$.

AX-CPT performance. Separate 2 (Age: younger, older) $\times 2$ (Mood induction: neutral, negative) univariate ANOVAs were conducted on errors and RTs for target trials. There were no main effects or interactions of mood induction for both errors and RTs, $p s>$.334. For non-target trials, 2 (Age: younger, older) $\times 2$ (Mood induction: neutral, negative) $\times 3$ (Trial type: control, probe-lure, cue-lure) mixed ANOVAs on error rates and RTs, separately, did not reveal any main effects or interactions of mood induction, $p s>$.312. Separate 2 (Age: younger, older) $\times 2$ (Mood 
induction: neutral, negative) univariate ANOVAs on the proactive indices for errors and RTs did not show main effects or interactions of mood induction either, $p s>.395$.

Together, these results suggest that the negative mood induction did not differentially change age differences in cognitive control patterns from those observed in the neutral induction group.

\section{Discussion}

Experiment 1B examined the effect of negative mood inductions on proactive and reactive control, as measured by the standard letter AX-CPT. Hypotheses were left open as this was the first study that examined the effect of induced negative moods on younger and older adults. Results revealed that the negative mood induction was successful at significantly lowering mood, relative to baseline. However, it should be noted that the average post-induction ratings fell within the mid-range of the scale. This suggests slightly more neutral than negative moods. There were no age differences in ratings after each induction. However, younger adults stayed in their post-induction mood throughout the time course whereas older adults' moods increased closer to baseline, consistent with evidence of faster regulation out of negative moods in older adults (Larcom \& Isaacowitz, 2009). Despite these differences, the negative mood induction appeared to be effective at temporarily lowering moods from baseline just before beginning each block of AX-CPT trials, based on post-induction ratings.

Using the standard letter AX-CPT, typical age-related patterns of control were found. When comparing the two age groups, younger adults made more cue-lure errors than older adults, indicative of greater proactive control. However, the two age groups made similar numbers of probe-lure errors. In the RT data, both age groups responded similarly to cue-lure trials, but older adults were slower on probe-lure trials (suggesting reactive control) than younger 
adults. Additionally, younger adults used proactive control as evident primarily in the RT data, but not in the error data. Older adults showed evidence of reactive control in the error rate data, but did not show a strong preference for either control mode in RTs. These results are consistent with the literature and are similar to those observed in Experiment 1A with neutral moods. A similar discrepancy between the error and RT data was also evident (see Discussion section of Experiment 1A). Together, these results suggest that induced negative mood had no evident effects on control patterns. These results are generally consistent with the limited number of studies on younger adults which also did not find any influence of negative emotion (from negative pictures presented on a trial-by-trial basis) on proactive and reactive control (e.g., Dreisbach, 2006). It extends these findings to induced negative mood and to an older adult sample.

Considering that studies have found evidence that positive mood can influence cognitive control (e.g., Chiew \& Braver, 2014; van Wouwe et al., 2011), how come negative moods do not seem to exert any influences (whether similar or different)? One possibility arises from the relationship between positive emotion and dopamine (Ashby, Isen, \& Turken, 1999). The DMC also predicts that dopamine serves a gating function that grants access to information in cognitive control (Braver \& Barch, 2002; Braver et al., 2007). As this relationship with dopamine is not present for negative emotions, some have hypothesized that only positive emotions will have an effect due to these dopaminergic connections (Dreisbach \& Goschke, 2004; Dreisbach, 2006; Mitchell \& Phillips, 2007). Thus, a lack of negative mood effects in Experiment 1B is consistent with the hypothesis that only positive mood will have effects on cognitive control. This relationship did not appear to differ between younger and older adults, despite evidence that induced negative moods can influence hypothesized component processes of cognitive control 
differently in the two age groups (e.g., Carpenter et al., 2013; Chu, 2014). As such, one of the goals of the next experiment was to examine the effect of positive mood inductions. 


\section{Chapter 5: Experiments 2A and 2B}

\section{Age Differences in Face-Letter AX-CPT under Neutral and Emotional Mood}

Experiment 1 revealed typical patterns of proactive control in younger adults and evidence of reactive control in older adults. However, induced negative mood did not have an effect on these control patterns. The literature review suggested that there is more evidence of effects due to induced positive versus neutral moods in younger adults (van Wouwe et al., 2011). However, a similar comparison has not been examined in older adults. Furthermore, it was possible that mood effects would be more apparent if task demands of the AX-CPT were reduced, based on patterns identified in the literature review (e.g., Borg et al., 2011; Carstensen et al., 2011; Truong \& Yang, 2014). Thus, Experiment 2 intended to examine the age differences in a modified and easier face-letter AX-CPT task under neutral (Experiment 2A) and emotional (negative and positive) mood (Experiment 2B) conditions.

\section{Experiment 2A: Age Differences in Face-Letter AX-CPT under Neutral Mood}

Objectives and rationale. The first purpose of Experiment $2 \mathrm{~A}$ was to examine whether proactive control could be promoted in older adults by making the task less demanding. To accomplish this, the letter cues in the standard letter AX-CPT were replaced with pictures of male and female faces. The validity of the cue was based on gender and was counterbalanced across two blocks (e.g., in one block, male faces were the valid cues and female faces were the invalid cues). This change was expected to reduce cognitive demands in several ways. First, compared to letter stimuli, face stimuli are more salient, externally valid, and likely to be meaningful to older adults (Kray et al., 2015; Phillips, Slessor, Bailey, \& Henry, 2014). This may help facilitate the maintenance of the contextual information represented by the face cues (i.e., the task rule of how to respond to the following probe). Second, use of different types of stimuli 
for cues versus probes (faces versus letters, respectively) creates a clear distinction between them (Reimer et al., 2015; van Wouwe et al., 2009). This may allow older adults to better use the valid face cues as predictive cues. Third, the use of gender distinctions generates a two-choice dichotomous rule that may be easier to keep in mind - this reduces the number of competitor choices (Kray et al., 2015). Alternatively though, it was also possible that having to make two decisions (first determine male/female, then determine the task rule) would make the task harder but could also promote deeper or more elaborative processing of the cue stimuli, as compared to when they were letters.

Neutral inductions were used again to keep procedures similar to that of Experiment 2A in order to provide a neutral baseline for comparison purposes. Results of this experiment were compared with that of Experiment $1 \mathrm{~A}$ which allowed for examinations of any changes that were due to the face-letter AX-CPT task specifically (relative to the letter AX-CPT), separate from the influences of induced negative and positive moods.

Hypotheses. Hypotheses focused on proactive control as the primary measure of interest. However, changes to proactive control were hypothesized to result in opposite effects to reactive control as well (i.e., an increase in one control mode may be accompanied by a decrease in the other). This was based on findings of enhanced proactive (poorer cue-lure performance) with reduced reactive control (better probe-lure performance) in older adults after strategy training and extended practice as compared to baseline levels (Braver et al., 2009; Paxton et al., 2006).

$\boldsymbol{H}$ 6: It was hypothesized that there would be less evidence of age differences in proactive control. It was hypothesized that the changes aimed at reducing the demands of the AX-CPT would result in less age differences between younger and older adults on proactive control (e.g., no age differences on probe-lure trials and/or similar proactive indices). 
$\boldsymbol{H}$ 7: Alternatively, it was also possible that the task change would result in comparable increases to proactive control in both younger and older adults. Thus, results would still reveal age differences.

H 8: Cross-experiment analyses with Experiment 1A (letter AX-CPT, neutral induction): It was hypothesized that results would be consistent with H6 - that is, it was hypothesized that measures of proactive control would show reduced age differences in Experiment 2A with the face-letter compared to the letter AX-CPT used in Experiment 1A.

\section{Experiment 2B: Age Differences in Face-Letter AX-CPT under Negative and Positive}

\section{Mood}

Objectives and rationale. Experiment 2A was hypothesized to reveal evidence of proactive control in older adults as measured on the face-letter AX-CPT task under neutral inductions. Thus, the intention was to use the same task in Experiment 2B to examine the effects of induced negative and positive moods in younger and older adults on cognitive control. This was based on the idea that mood effects may be more apparent when the AX-CPT task was less resource-demanding (Borg et al., 2011; Carpenter et al., 2013; Phillips et al., 2002; Truong \& Yang, 2014). Therefore, younger and older participants in Experiment 2B received either a negative or positive mood induction prior to completing the face-letter AX-CPT consisting of face and letter stimuli. Positive mood inductions were included in Experiment 2B to address the open question of the impact of positive mood on older adults' cognitive control. It was possible that older adults under positive mood may show different effects as compared to negative and neutral moods (Biss et al., 2012; Carpenter et al., 2013; Noh et al., 2012). Additionally, prior studies with younger adults have found discrepant findings of reduced (van Wouwe et al., 2011) and enhanced proactive control (Chiew \& Braver, 2014) under induced positive compared to 
neutral moods. Thus, Experiment 2B aimed to contribute to this literature with both younger and older adult samples. Additionally, the effects of induced negative moods have not been compared to effects of induced positive mood; thus, participants received either negative or positive mood inductions in this experiment. Cross-experiment analyses with Experiment 2A were also conducted to compare the effects of induced negative and positive versus neutral moods. Analyses also compared results from Experiment 1B to examine whether any differences would be found when performance on the two AX-CPT versions were compared under neutral versus negative moods.

Hypotheses. As no studies have examined the effect of induced negative moods and positive moods on proactive and reactive control in both younger and older adults within a study, it was an open question as to what patterns would emerge. Overall, it was hypothesized that mood effects would be more apparent on the face-letter than letter version of the AX-CPT, as measured by cross-experiment analyses with Experiment 1B. Several competing hypotheses were proposed, as described below.

$\boldsymbol{H}$ 9: It was hypothesized that induced negative mood would not have an effect on younger adults’ cognitive control (Dreisbach, 2006; Fröber \& Dreisbach, 2012). For older adults, it was not clear whether effects of negative mood would change their typical proactive control impairments. However, based on findings from Experiment 1B, an effect of negative mood on cognitive control in older adults was not expected either. Alternatively, it was possible that the change to a less demanding AX-CPT task may allow more evident effects of induced mood to emerge. Thus, the hypotheses for the directionality of these effects remained open.

$\boldsymbol{H}$ 10: Under induced positive mood, it was hypothesized that younger adults would show evidence of reduced proactive control, relative to negative mood. This was based on similar 
findings as reviewed in the Introduction (Dreisbach, 2006; Fröber \& Dreisbach, 2012). For older adults, it was unclear what effects would be seen, as studies have shown both facilitative (Carpenter et al., 2013; Chu, 2014; Noh et al., 2012) and detrimental effects (Phillips et al., 2002) of positive mood on components of cognitive control in older adults (e.g., planning, Phillips). However, based on findings that positive mood is related to greater orienting to cues (Noh et al., 2012) and working memory maintenance (Carpenter et al., 2013), it was possible that positive moods may enhance proactive control specifically. 


\section{Experiment 2A: Age Differences in Face-Letter AX-CPT under Neutral Mood}

Participants in Experiment 2A received a neutral mood induction prior to completing the face-letter AX-CPT task with faces as cues and letters as probes.

\section{Method}

\section{Participants}

Participants were 35 healthy younger (ages 18-28) and 26 healthy older adults (ages 6587). Six younger adults were excluded due to high DASS scores that were classified as “extremely severe”. One younger and one older adult were excluded due to a prior stroke and brain surgery, respectively. One younger adult had current diagnoses of depression and anxiety. The final sample included in the analyses was 27 younger and 25 older adults (Table 4). 
Table 4

Participant Demographic Characteristics and Cognitive Performance

\begin{tabular}{|c|c|c|c|c|c|c|}
\hline \multirow[t]{2}{*}{ Characteristic } & \multicolumn{2}{|c|}{$\begin{array}{l}\text { Younger Adults } \\
(n=27)\end{array}$} & \multicolumn{2}{|c|}{$\begin{array}{l}\text { Older Adults } \\
(n=25)\end{array}$} & \multirow[t]{2}{*}{$p$-value } & \multirow[t]{2}{*}{$d$} \\
\hline & $M$ & $(S D)$ & $M$ & $(S D)$ & & \\
\hline Age & 20.63 & $(3.00)$ & 74.04 & $(6.84)$ & $<.001^{* *}$ & 10.11 \\
\hline Female/male (ratio) & $22 / 5$ & & $20 / 5$ & & $.892^{x}$ & \\
\hline Years of education & 14.07 & $(1.67)$ & 16.94 & (3.04) & $<.001^{* *}$ & 1.17 \\
\hline Health rating & 7.82 & (1.39) & 8.25 & (1.11) & .228 & 0.34 \\
\hline FTP & 51.78 & $(8.47)$ & 38.12 & (7.39) & $<.001^{* *}$ & 1.72 \\
\hline PANAS-Positive & 24.74 & $(5.47)$ & 35.00 & (5.59) & $<.001^{* *}$ & 1.86 \\
\hline PANAS-Negative & 14.59 & (3.43) & 12.28 & (2.97) & $.013^{*}$ & 0.72 \\
\hline DASS-Depression & 7.00 & $(6.48)$ & 4.58 & $(4.66)$ & .140 & 0.43 \\
\hline DASS-Anxiety & 8.00 & (5.38) & 2.56 & (3.98) & $<.001^{* *}$ & 1.15 \\
\hline Corsi & 0.50 & $(0.14)$ & 0.25 & $(0.14)$ & $<.001 * *$ & 1.79 \\
\hline Pattern comparison & 71.56 & $(14.38)$ & 45.32 & $(9.53)$ & $<.001 * *$ & 2.15 \\
\hline DCCST & 8.84 & (1.19) & 7.75 & $(0.84)$ & $<.001^{* *}$ & 1.06 \\
\hline Flanker & 9.42 & $(0.52)$ & 8.53 & $(0.53)$ & $<.001^{* *}$ & 1.70 \\
\hline Vocabulary & 1475.22 & (217.17) & 1925.56 & (250.78) & $<.001^{* *}$ & 1.92 \\
\hline SBT & & & 0.40 & $(0.20)$ & & \\
\hline
\end{tabular}

Note . Education = average number of years of formal education; health rating = report of self-rated health on a scale from 1 (poor) to 10 (excellent); FTP = Future Time Perspective Scale; PANAS-Positive/Negative = Positive Affect Negative Affect Schedule; DASS-Depression/Anxiety = Depression, Anxiety, and Stress Scales; Corsi = Corsi Block; Pattern comparison $=$ Pattern Comparison Processing Speed Test; DCCST $=$ Dimensional Change Card Sort Test; Flanker $=$ Flanker Inhibitory Control and Attention Test; Vocabulary = Picture Vocabulary Test; SBT = Short Blessed Test

${ }^{*} p<.05 .{ }^{* *} p<.001 .{ }^{x}$ denotes the use of chi-square statistic; all other analyses used independent $t$-tests $d=$ Cohen's $d$ effect size calculation for between subjects 


\section{Face-Letter AX-CPT Task}

This version of the AX-CPT task modified the letter version by replacing cue letters with neutral female and male faces. Probe stimuli were the same as those used in the letter AX-CPT task. This version was used in both studies of Experiment 2.

Stimuli. All face stimuli were obtained from the FACES collection (Ebner, Riediger, \& Lindenberger, 2010), a validated database developed for research purposes, containing photographs of naturalistic Caucasian adult faces (ages 19-80) displaying neutral and emotional expressions. All face models were photographed wearing the same gray t-shirt. The images were colour photographs taken against gray backgrounds. Each image was $335 \times 419$ pixels and was displayed centrally against a black background on the computer screen. Faces in this database have been rated for perceived facial expression in a validation study with $N=154$ raters (Ebner et al., 2010). This data consisted of the average rater identification accuracy, which was the percentage of raters who were able to correctly identify the intended emotional expression displayed by the face. This data was used to select the subset of neutral faces used as stimuli in the face-letter AX-CPT. A subset of 32 images were selected for the face-letter AX-CPT task and consisted of 16 younger (ages: 19-31) and older (ages 69-80) male and female faces displaying neutral expressions (two images per person) ${ }^{10}$. Faces selected were those in which the gender of the face was easy to distinguish in addition to receiving relatively high average rater identification for their neutral face. The faces that served as valid and invalid cue images in each block were equally distributed by age group (younger or older) of the face model. The rater recognition accuracies from the validation study are presented in Table 5.

\footnotetext{
${ }^{10}$ Image codes of selected subset: younger males $(016,153,160,167)$ and females $(028,048,069$, 182); older males $(004,018,027,166)$ and females $(012,030,060,079)$.
} 
Table 5

Rater Accuracies for the Emotional Expression of Selected Faces

\begin{tabular}{lllll}
\hline \multirow{2}{*}{ Rater Accuracies } & \multicolumn{2}{c}{ Male faces } & \multicolumn{2}{c}{ Female faces } \\
\cline { 2 - 5 } & Younger faces & Older faces & Younger faces & Older faces \\
\hline Mean (SD) & $91.25(5.31)$ & $81.00(11.77)$ & $94.00(3.51)$ & $87.63(7.19)$ \\
Range & $83 \%-99 \%$ & $67 \%-96 \%$ & $89 \%-99 \%$ & $74 \%-98 \%$ \\
\hline
\end{tabular}

Note. Mean scores refer to the percentage of raters from the validation study who accurately identified the emotional expression displayed by the face.

Trial procedure and types. The trial procedure was identical to that of the letter AX-CPT task. The only difference was that participants were required to press the spacebar key (labelled "FACE”) with their thumb to each cue, instead of a "non-target” response.

The trial types were structured identically to that of the letter AX-CPT task, with only a change in the cue stimuli used (faces instead of letters). The specification for a valid-cue varied by block and was based on gender classification. The valid cues were male faces in one block and female faces in the other block. The order of the blocks was counterbalanced across participants. In the valid-male block, male faces served as valid-cues and female faces as invalidcues (Figure 10). Thus, a male face (valid-cue) followed by the letter X (valid-probe) required a target response in this block. In the valid-female block, female faces were the valid-cues and male faces were the invalid-cues. Cue-lure trials consisted of a male/female valid cue face followed by an invalid-probe letter. Probe-lure trials consisted of a male/female invalid-cue face followed by the valid-probe letter $\mathrm{X}$. Control trials consisted of a male/female invalid-cue face followed by an invalid-probe letter. 


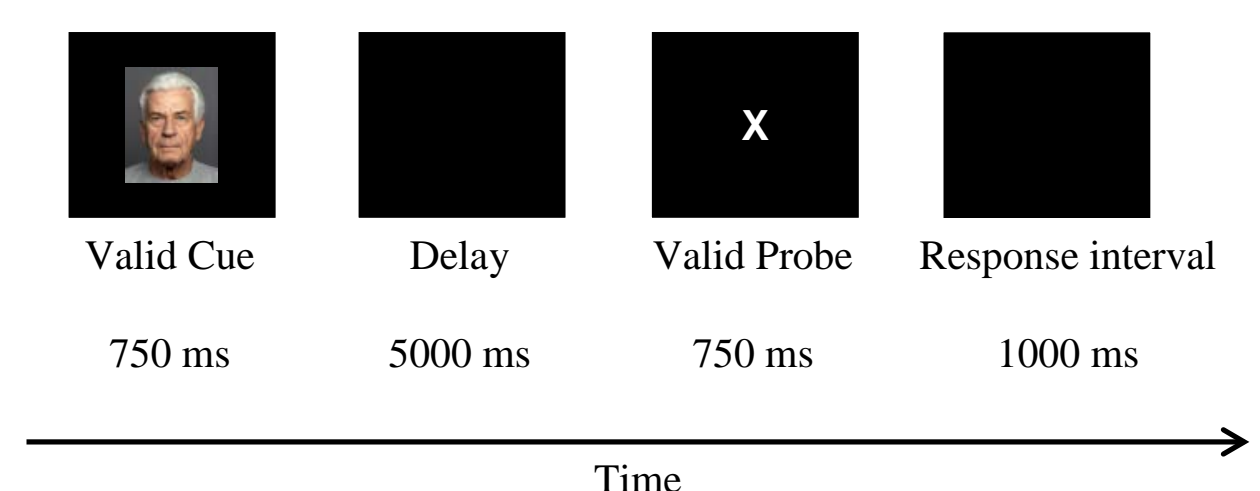

Time

Figure 10. Sample illustration of a target trial in the male block: male face (valid cue) followed by letter $\mathrm{X}$ (valid probe).

Task procedure. The procedure was identical to the letter AX-CPT task except that the instructions specified which gender (male/female) served as the valid-cue face in the first block. For the second block, task instructions specified that the opposite gender now served as the valid-cue face. Participants received additional practice with this new task rule.

\section{Procedure}

The procedure was the same as Experiment 1.

\section{Results}

\section{Mood Ratings and Induction Questionnaire}

Valence. The mixed ANOVA on SAM valence ratings revealed a significant main effect of time point, $F(3.34,166.95)=10.97, p<.001, \eta_{\mathrm{p}}{ }^{2}=.18$. There was also a main effect of age, $F(1,50)=8.17, p=.006, \eta_{\mathrm{p}}{ }^{2}=.14$, with lower ratings reported by younger than older adults. These effects were qualified by an age by time point interaction, $F(5,250)=2.84, p=.016, \eta_{\mathrm{p}}{ }^{2}=$ .05. For older adults, there was a trend toward a main effect of time point that did not reach significance, $F(2.22,53.22)=2.67, p=.073, \eta_{\mathrm{p}}{ }^{2}=.10$. After Bonferroni corrections, the only comparisons that remained significant were the differences after the first induction (i.e., post- 
induction 1) and after the second AX-CPT block (i.e., post-block 2), with lower ratings in the latter, $p=.002$. For younger adults, the main effect of time point was significant, $F(2.88,74.78)$ $=13.20, p<.001, \eta_{\mathrm{p}}{ }^{2}=.34$. Multiple comparisons revealed that ratings were higher at baseline than subsequent time points, $p$ s $<.002$, except after the first induction, $p=.068$. Ratings at the first and second post-induction time points did not differ, $p=.026$, nor did the subsequent time points differ from each other, $p s>.009$ (Figure 11, left panel).

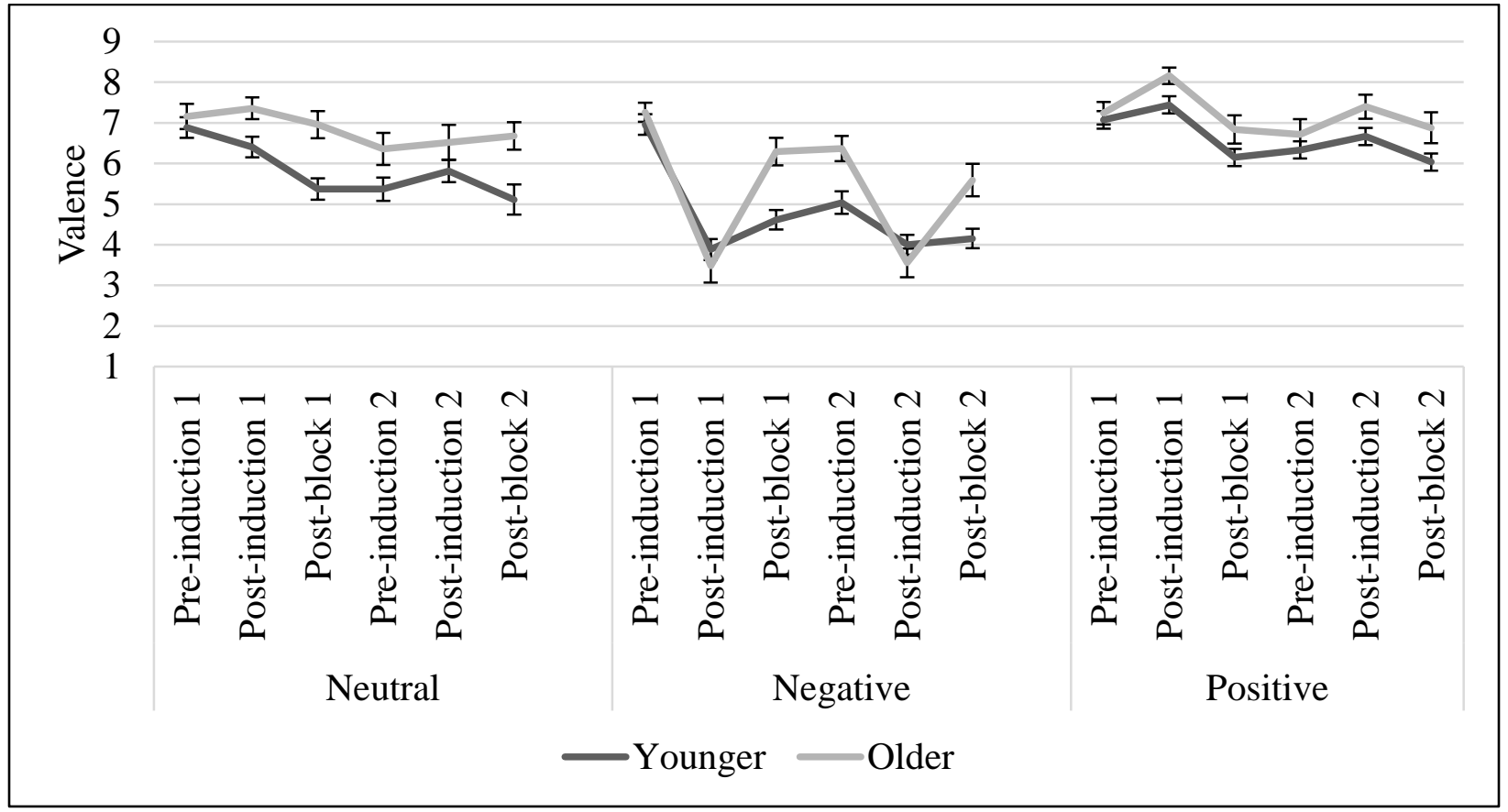

Figure 11. Mean valence ratings of mood across time point; Left panel: neutral mood induction (Experiment 2A); middle panel: negative mood induction (Experiment 2B); right panel: positive mood induction (Experiment 2B). Error bars denote standard error.

Arousal. The mixed ANOVA on SAM arousal ratings also revealed a main effect of time point, $F(3.24,161.93)=7.79, p<.001, \eta_{\mathrm{p}}^{2}=.14$, with simple contrasts showing lower arousal ratings compared to baseline for all subsequent time points, $p s<.032$. All other comparisons 
were not significant after Bonferroni adjustments, $p \mathrm{~s}>$.005. There was no effect of age group or an age by time point interaction, $p s>.604$ (Figure 12, left panel).

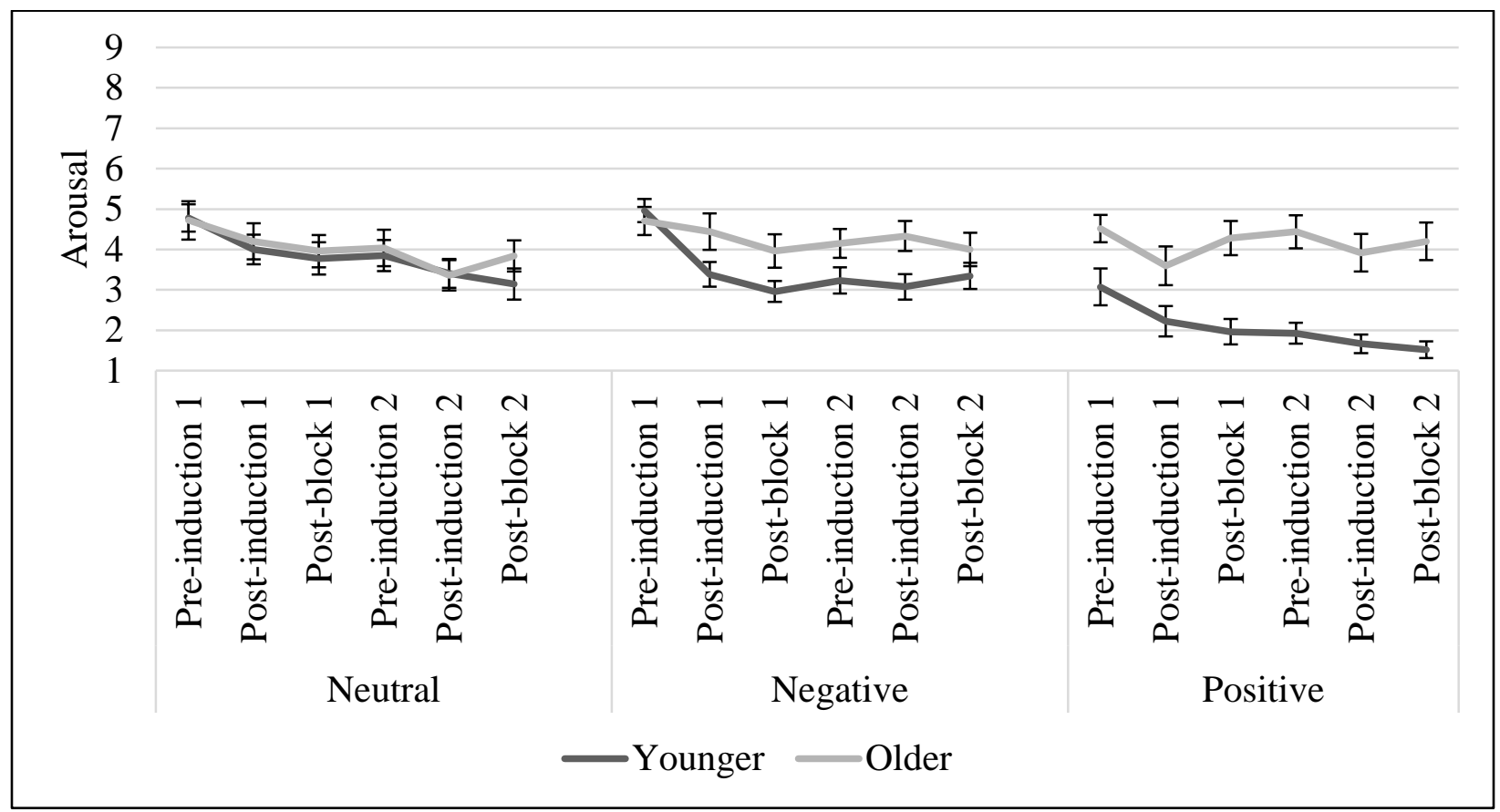

Figure 12. Mean arousal ratings of mood across time point; Left panel: neutral mood induction (Experiment 2A); middle panel: negative mood induction (Experiment 2B); right panel: positive mood induction (Experiment 2B). Error bars denote standard error.

Induction questionnaire. Responses to each question were analyzed with $t$-tests which revealed no age differences for the familiarity of the sounds heard, $p=.118$, or ratings of induction effectiveness, $p=.364$. For the question on slideshow processing, $76.92 \%$ indicated that they were paying attention, $11.54 \%$ indicated they were trying to memorize, and $11.54 \%$ indicated they were doing neither. To examine for age differences in these responses, chi-square analyses were conducted for each category of processing. These analyses revealed no age differences for each category, $\chi^{2} s<0.59$, $p s>.442$. No further analyses were conducted on the induction questionnaire data. 
In summary, the neutral mood induction was somewhat effective as the mood at the two post-induction time points was maintained as compared to the two corresponding pre-induction time points. Consistent with Experiment 1A, there was a slight reduction in mood ratings (but still within a neutral mood range) across time points (compared to baseline), particularly for young adults and following each AX-CPT block, and participants maintained these relatively neutral moods across time.

\section{AX-CPT Performance}

As in Experiment 1, the results (error rates and RTs) were analyzed separately for target and non-target trials. Only response time data for correct responses were used (94.41\% of all trials). One younger and one older adult did not have any correct responses for probe-lure trials and as such, no correct response time data for that trial type.

\section{Target trials.}

Error rates. Younger adults $(M=0.05, S D=0.05)$ had higher error rates for target trials than older adults ( $M=0.02, S D=0.02), t(36.77)=2.55), p=.015$ (Figure 13, left panel). 


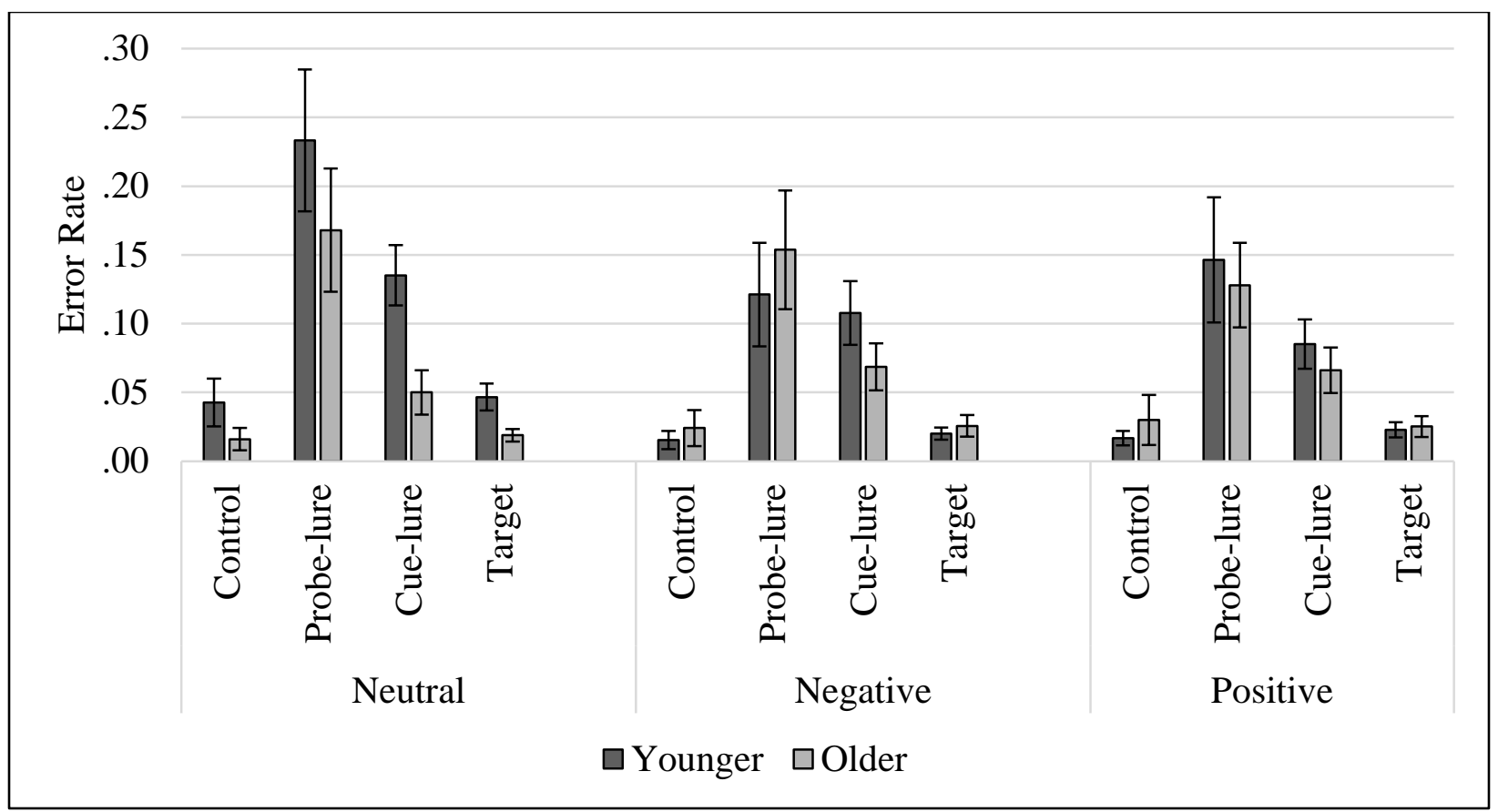

Figure 13. Mean error rates by trial type; Left panel: neutral mood induction (Experiment 2A), middle panel: negative mood induction (Experiment 2B); right panel: positive mood induction (Experiment 2B). Error bars denote standard error.

Response times. Younger $(M=-0.19, S D=0.14)$ and older $(M=-0.22, S D=0.15)$ adults did not differ on response times for target trials, $t(50)=.78, p=.437$ (Figure 14, left panel). 


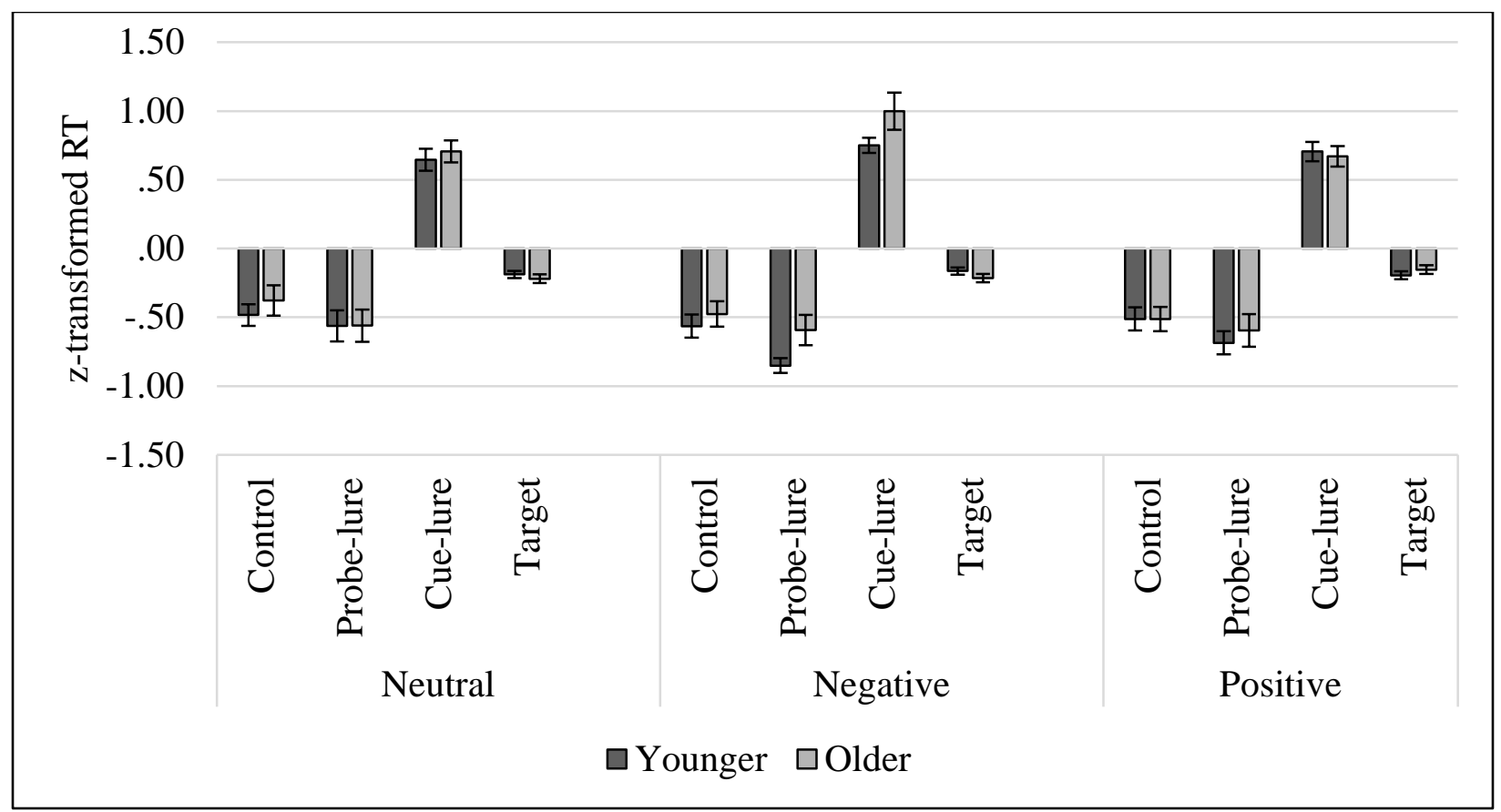

Figure 14. Mean z-transformed RTs by trial type; Left panel: neutral mood induction (Experiment 2A), middle panel: negative mood induction (Experiment 2B); right panel: positive mood induction (Experiment 2B). Error bars denote standard error.

\section{Non-target trials.}

Error rates. There was a main effect of trial type, $F(1.32,65.77)=16.32, p<.001, \eta_{\mathrm{p}}{ }^{2}=$ .25, with planned contrasts revealing higher error rates for probe- and cue-lure than control trials, ps $<.001$, as well as higher error rates for probe- than cue-lure trials, $p=.004$. There was also a main effect of age, $F(1,50)=4.76, p=.034, \eta_{p}{ }^{2}=.09$, with higher error rates for younger than older adults. The age by trial type interaction was not significant, $p=.619$. However, paired samples $t$-tests for each age group separately revealed that error rates were higher for probe- ( $M$ $=0.17, S D=0.22)$ than cue-lure $(M=0.05, S D=0.08)$ trials for older adults, $p=.009$ but were equivalent for younger adults $\left(M_{\text {probe-lure }}=0.23, S D_{\text {probe-lure }}=0.27 ; M_{\text {cue-lure }}=0.14, S D_{\text {cue-lure }}=\right.$ 0.11), $p=.104$. Additionally, both age groups had equivalent performance on control $\left(M_{\text {younger }}=\right.$ 
$\left.0.04, S D_{\text {younger }}=0.09 ; M_{\text {older }}=0.02, S D_{\text {older }}=0.04\right)$, and probe-lure trials, $p s>.183$. However, for cue-lure trials, younger adults had higher error rates than older adults, $p=.031$ (Figure 13, left panel).

Response times. There was a main effect of trial type, $F(2,96)=117.46, p<.001, \eta_{\mathrm{p}}{ }^{2}=$ .71. Compared to cue-lure trials, responses were faster to both control and probe-lure trials, $p s<$ .001 , which did not differ from each other, $p=.30$. There was no effect of age, $p=.690$, or an age by trial interaction, $p=.928$. The lack of an interaction suggests that both age groups performed equivalently on all trial types. In support of this, planned contrasts revealed that there were no age differences for any of the trials, $p \mathrm{~s}>.434$. Additionally, both age groups responded slower to cue-lure than probe-lure and control trials, $p s<.001$.

Proactive index. For younger adults, the proactive index for errors $(M=-0.05, S D=$ 0.68) was not different than zero, $t(26)=-0.40, p=.695$, but it was significantly lower than zero for older adults $(M=-0.48, S D=0.48), t(24)=-5.01, p<.001$. Older adults had lower proactive index values for errors than younger adults, $F(1,51)=6.83, p=.012, \eta_{\mathrm{p}}{ }^{2}=.120$ (Figure 15, left panel). 


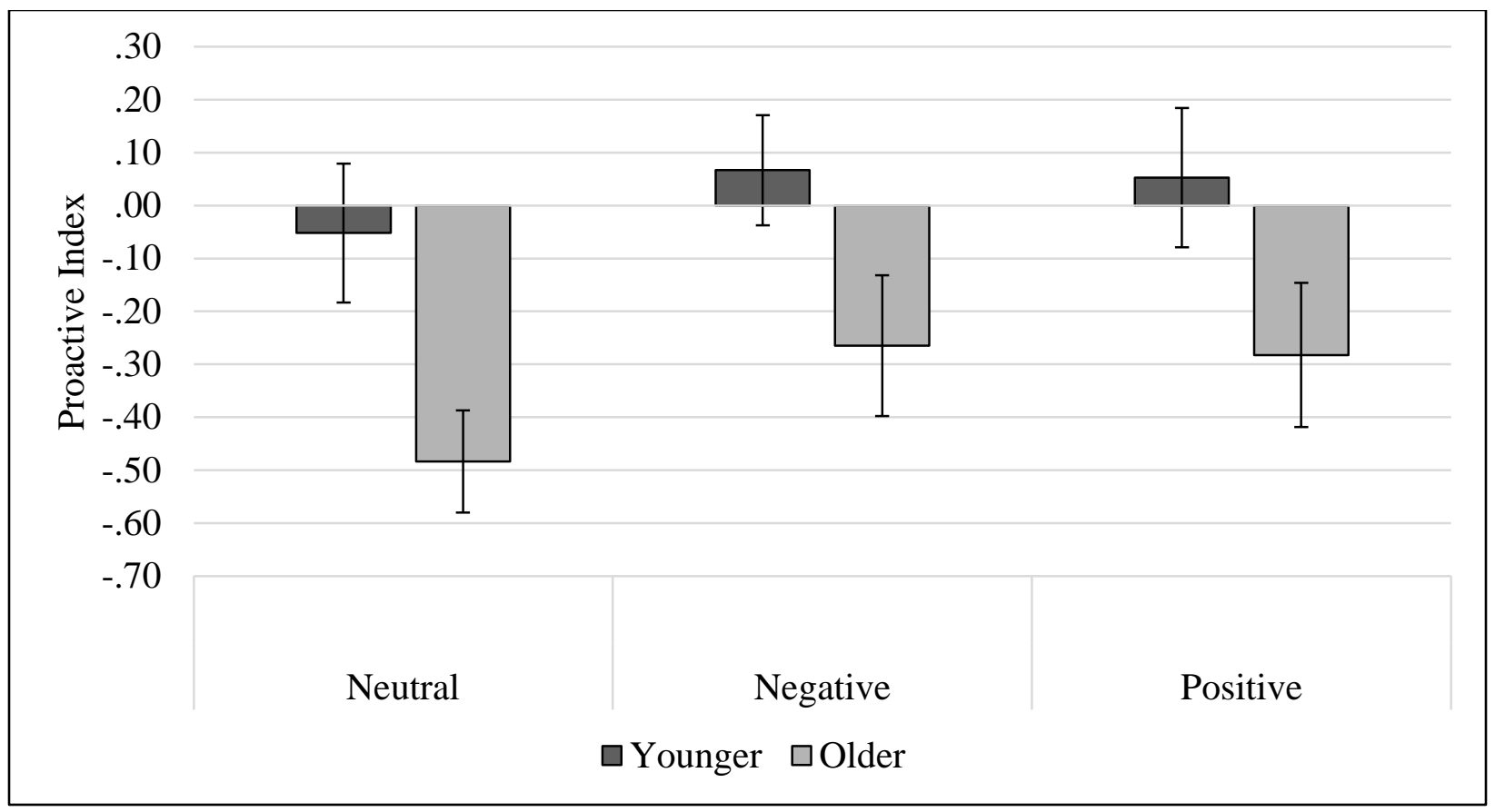

Figure 15. Mean proactive index for errors; Left panel: neutral mood induction (Experiment 2A), middle panel: negative mood induction (Experiment 2B); right panel: positive mood induction (Experiment 2B). Error bars denote standard error.

The proactive index for RTs was significantly greater than zero in both younger $(M=$ $0.20, S D=0.13)$ and older $(M=0.18, S D=0.11)$ adults, $p s<.001$, and there was no age group difference, $p=.665$ (Figure 16, left panel). 


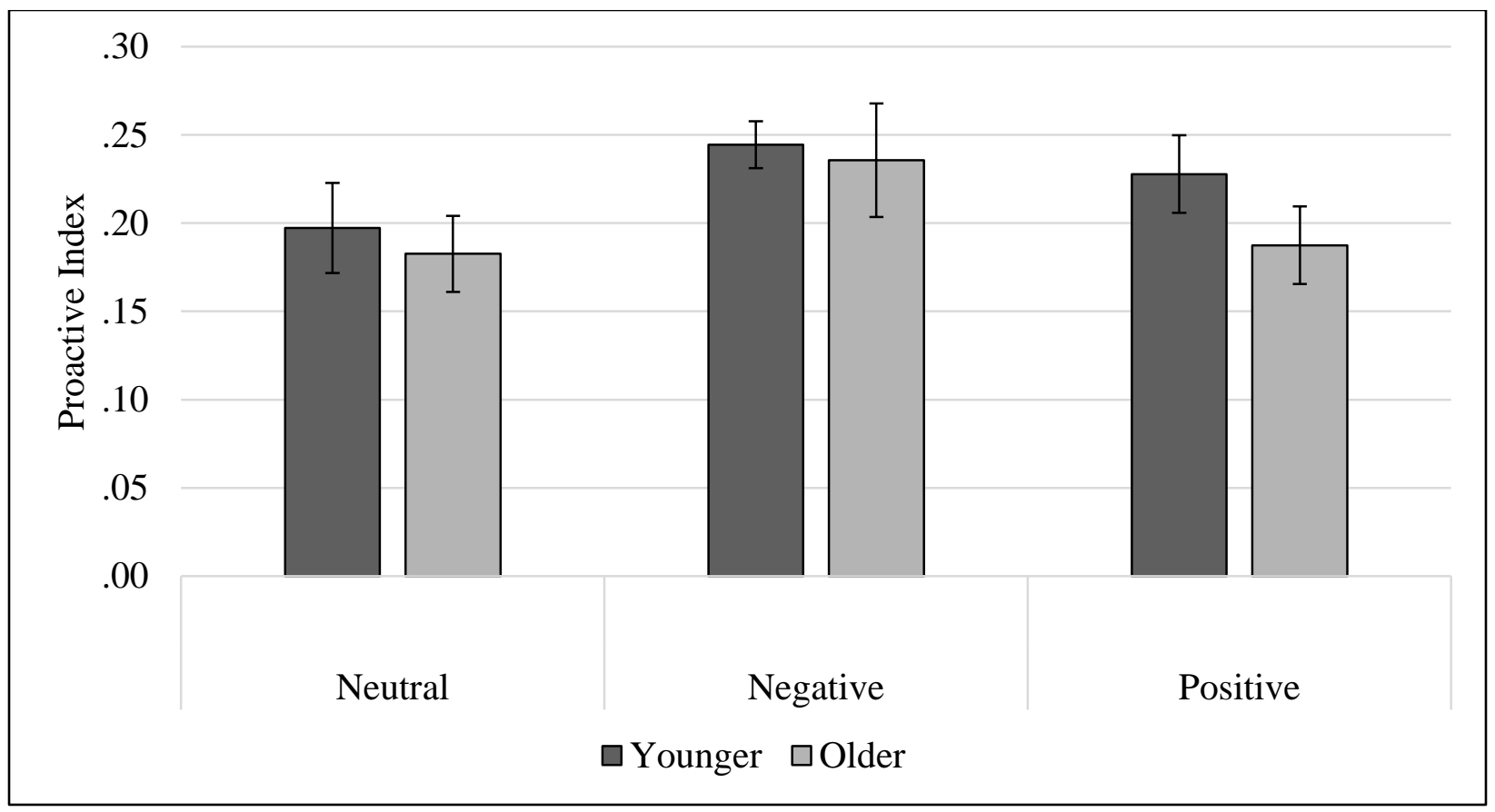

Figure 16. Mean proactive index for RTs; Left panel: neutral mood induction (Experiment 2A), middle panel: negative mood induction (Experiment 2B); right panel: positive mood induction (Experiment 2B). Error bars denote standard error.

Together, the data present very different patterns than those observed in Experiment 1, particularly in the RT data. The evidence suggests reduced reactive control (faster probe-lure responses) and an increase in proactive control (slower cue-lure responses). This is seen in both age groups, resulting in proactive indices for RTs that do not differ in younger and older adults. This is in contrast to Experiment 1 in which older adults show more evidence of reactive than proactive control.

\section{Individual Differences Measures}

As in previous experiments, variables that showed age differences were correlated with the proactive index for errors, which also showed a significant age difference (larger proactive index for younger than older adults). The following measures correlated with the error index: 
FTP, $r(52)=.32, p=.022$; pattern comparison, $r(52)=.33, p=.017$; DCCST, $r(51)=.29, p=$ .039 , and vocabulary, $r(55)=-.29, p=.034$. Including these variables in the ANCOVA on proactive index in errors revealed that the effect of age was no longer significant, $p=.875$, and all other effects were not significant, $F \mathrm{~s}<.807, p s>.374$.

\section{Cross-Experiment (1A vs. 2A) Analyses}

There appeared to be more proactive tendencies in both age groups in Experiment 2A compared to $1 \mathrm{~A}$, likely driven by modifications to the AX-CPT task. In order to further examine these effects, cross-experiment analyses with Experiment 1A, both of which involved neutral mood inductions, were conducted to compare performance on the two versions of the AX-CPT task: letter versus face-letter.

Mood ratings. $t$-tests were conducted to check whether induced moods were similar across the two experiments. These results revealed lower valence ratings for Experiment $1 \mathrm{~A}$ than 2A that were significant at baseline, $p=.016$, marginally significant after the first induction, $p=$ .052 , and significant after the first AX-CPT block. No other differences were found, $p s>.352$. The same comparisons conducted on the arousal ratings revealed no differences between experiments, $p \mathrm{~s}>.060$.

\section{AX-CPT performance.}

Target trials. A 2 (Age: younger, older) × 2 (AX-CPT version: letter, face-letter) univariate ANOVA on the error data for target trials revealed a main effect of version, $F(1,105)$ $=9.62, p=.002, \eta_{\mathrm{p}}{ }^{2}=.09$, with lower error rates on the face-letter than letter version. All other effects were not significant, $F \mathrm{~s}<.2 .42$, $p \mathrm{~s}>.123$. The same analysis was conducted for RTs. The main effect of version was significant, $F(1,105)=40.14, p<.001, \eta_{\mathrm{p}}{ }^{2}=.28$, with faster responses in the letter than face-letter version. The age by version interaction was approaching 
significance, $F(1,105)=3.72, p=.057, \eta_{\mathrm{p}}{ }^{2}=.04$. This interaction was driven by age differences in the letter version, $p=.040$, but not the face-letter version, $p=.437$, with slower responding in younger $(M=-0.33, S D=0.11)$ than older $(M=-0.40, S D=0.12)$ adults. The 3-way interaction was not significant, $p=.492$. Decreased target errors but increased target response times in the face-letter versus letter version suggest a potential speed-accuracy trade off, with greater prioritization of accuracy over speed.

\section{Non-target trials.}

A 2 (Age: younger, older) $\times 2$ (AX-CPT version: letter, face-letter) $\times 3$ (Trial type: control, probe-lure, cue-lure) mixed ANOVA on the non-target error rates revealed a significant main effect of trial type, $F(1.22,124.74)=31.93, p<.001, \eta_{\mathrm{p}}{ }^{2}=.24$. Error rates were lower for control than interference trials, $p<.001$, and were lower for cue- than probe-lure trials, $p<.001$. The main effect of age was approaching significance, $F(1,102)=3.80, p=.054, \eta_{\mathrm{p}}{ }^{2}=.04$, with higher error rates in younger than older adults. The main effect of version was trending significance, $F(1,102)=3.08, p=.082, \eta_{\mathrm{p}}{ }^{2}=.03$, with higher error rates in the face-letter than letter version. All interactions were not significant, Fs <.33, ps $>.169$.

The same mixed ANOVA was conducted on the RT data, which revealed main effects of version and trial type, $F \mathrm{~s}>45.60, p \mathrm{~s}<.001, \eta_{\mathrm{p}}{ }^{2} \mathrm{~s}>.45$. These were qualified by age by trial type, version by trial type, and age by version by trial type interactions, $F \mathrm{~s}>4.25$, $p \mathrm{~s}<.016, \eta_{\mathrm{p}}{ }^{2} \mathrm{~s}>$ .04. To simplify interpretation of the 3-way interaction, and to specifically compare proactive control use in the two versions, the proactive indices for RTs were analyzed with a 2 (Age) $\times 2$ (Version) univariate ANOVA. This revealed main effects of age and version, Fs $>5.49$, ps < $.021, \eta_{\mathrm{p}}{ }^{2} \mathrm{~s}>.05$. The age by version interaction was trending significance, $F(1,102)=3.04, p=$ $.084, \eta_{\mathrm{p}}{ }^{2}=.03$. As reported earlier, younger adults were more proactive than older adults in the 
letter version, but there were no age differences in the face-letter version. This was driven by an increase in proactive control in both age groups in the face-letter compared to the letter version, ps $<.001$.

As confirmed by the cross-experiment analyses, the face-letter version appeared to facilitate proactive control, at least in the RTs, to a greater extent than the letter version. This proactive advantage was found for both younger and older adults. For younger adults, the increase in proactive control was mainly driven by faster probe-lure responses in the face-letter than letter version, with no change to the cue-lure trials. For older adults, the increase in proactive control (best reflected in the proactive index for RTs) was driven by both faster probelure responses and slower cue-lure responses in the face-letter versus letter version. However, it should be noted that these effects were only evident in the RT, but not the error data.

\section{Discussion}

Experiment 2A used a modified AX-CPT with faces as cue stimuli (face-letter AX-CPT), alongside a neutral mood induction. This experiment served to test the effects of changing the cue stimuli from multiple-choice alphabet letters to two-option human male and female faces. Neutral mood inductions were included to match the conditions of Experiment 1A in order to facilitate cross-experiment analyses. Similar to Experiment 1A, these inductions resulted in mood ratings that were in a fairly neutral valence range (4-6), particularly for younger adults. In contrast, older adults reported slightly more positive ratings relative to younger adults and their moods tended to be more stable across time points.

Experiment 2A provided evidence that changes to the standard letter AX-CPT (i.e., using faces instead of letters as cues) can change age-related patterns of control. Performance on the face-letter AX-CPT revealed no significant age by trial type interactions observed in either the 
error or RT data. This supported the hypothesis (H 6) that age differences in proactive control would be reduced using the face-letter AX-CPT. To more directly assess this change, crossexperiment analyses comparing Experiment 1A and 2A were conducted, which differed only on the AX-CPT version used (letter- vs. face-letter, respectively). These analyses revealed a shift toward proactive control in RTs in both age groups, as evidenced by a larger proactive index, in the face-letter versus letter AX-CPT. For younger adults, this increase in the proactive index was primarily driven by faster responding to probe-lure trials with no changes in RTs on cue-lure trials across experiments. For older adults, however, the increase in the proactive index was due to both faster responding on probe-lure trials coupled with slower responding on cue-lure trials in the face-letter versus letter AX-CPT. This asymmetry is not accounted for by generalized speeding or slowing of responses in the face-letter AX-CPT. Thus, the modifications of the faceletter AX-CPT resulted in performance shifts that led older adults to perform much more similarly to younger adults.

However, it should be noted that planned contrasts on the error data still showed evidence of reduced proactive and greater reactive control in older than younger adults. This was demonstrated in a proactive index that did not significantly differ from zero for younger adults, but was significantly less than zero for older adults, indicative of more reactive control. The same planned contrasts on the RT data did not reveal any age differences. Interestingly, the reactive tendency reflected in errors (i.e., higher errors for probe- than cue-lure trials) made by older adults appears to be a consistent finding across results of all the experiments thus far. This suggests that while changes to the AX-CPT appear to have substantial effects on RTs, it did not substantially change error rates. Thus, it appears that the face-letter AX-CPT resulted in a possible prioritization of faster responses on invalid cue trials (control and probe-lure) but slower 
valid cue responses. Aside from a possible speed-accuracy trade-off for target trials (reduced errors but slowed responses), faster responses were not accompanied by increases in errors for the non-target trials. This suggests that the results may not be fully accounted by a speedaccuracy trade-off. The same patterns were found in younger adults as well, suggesting that the change in task had equivalent effects on both age groups. Interestingly, only older adults exhibited an increase in cue-lure trials, possibly suggesting that they were now more cuefocused, as compared to when measured on the letter AX-CPT.

Nevertheless, the results of the current experiment and analyses with Experiment $1 \mathrm{~A}$ demonstrate that it is possible to enhance proactive control in older adults through modifications of the standard AX-CPT. This was accomplished by changing the cue stimuli from letters to faces. Why did this modification facilitate proactive control? There are several possibilities. First, reduced error rates on target trials support the hypothesis that the change from letter to face cues can facilitate cue maintenance, making it easier to prepare a target response after a valid cue. This may have occurred because the face cue was more distinct or salient than the simple letter A in the standard AX-CPT. As face cues are very different from letter probes, this marked distinction may have made it easier to distinguish between a cue and probe and could possibly direct relatively greater processing towards the cue and away from the probe. This distinction could have helped participants quickly and correctly prepare the appropriate response (target or non-target) after detection of the target gender face cue, in anticipation of the upcoming letter probe. Some support for this explanation comes from a study finding increased proactive control after training (relative to baseline) that was aimed at cue processing, with strategy focused on teaching older adults how to use the cue to effectively prepare for upcoming probes (Paxton et al., 2006). It should be noted that increased proactive control was also found in two control 
groups that received either reinstruction of task goals or additional practice, indicating that the strategic training was likely not the most critical factor. However, there was some evidence of slightly greater proactive control in the training than control groups, suggesting greater benefits from strategic training focused on cue processing.

Another explanation of enhanced proactive control in the current experiment arises from the reduction of potential items belonging to the stimulus set that could serve as valid versus invalid cues in the face-letter compared to the letter AX-CPT. In both versions, the basic task rule is the same: respond "target" to the letter X if the preceding item is a valid cue and "nontarget” if it is an invalid cue. In the face-letter AX-CPT, an invalid cue is based on gender (e.g., any female out of the eight unique female faces that served as invalid cues). In the AX-CPT, the invalid cue was any non-A letter, out of a possible 22 letters. Additionally, some letters that served as invalid cues also served as invalid probes. However, in the face-letter version, they are mutually exclusive (e.g., all cues were female faces and all probes were letters). As such, the number of possible items that could serve as invalid cues was reduced in the face-letter version. This is hypothesized to have reduced interference and may have made the overall task easier, thus promoting proactive control. Supporting evidence of this explanation comes from a study that had a significantly reduced number of competitive cues and probes in two variants of the AX-CPT and also found evidence of proactive control in older adults (Kray et al., 2015). This possibility was further investigated in Experiment 3 and informed the design of the face AXCPT, which attempted to match the number of competitive invalid cues and probes to that of the letter AX-CPT.

Additionally, it is also possible that faces, which are more meaningful, salient, and detailed than letters, may have facilitated richer goal representations. This could directly aid goal 
processing and maintenance, and would be consistent with the strong patterns of proactive control observed in this experiment, which appeared to be driven by enhanced cue-related processing. Indeed, this is most evident in the RT data for older adults. As compared to the standard AX-CPT with letter cues, older adults were significantly faster for all invalid cue trials - they were able to maintain and use the invalid face cue to appropriately prepare a non-target response, enabling faster responses. In contrast, they were slower for all valid cue trials (target and cue-lure). This pattern suggests that older adults were actively maintaining and using the valid face cue to prepare an appropriate response, which resulted in slower responses on cue-lure trials. This advance preparation should have also quickened target responses. Instead, it appears that older adults may have prioritized accuracy for target trials, as responses were more accurate, but slower, in the face-letter than letter AX-CPT. These possibilities were further examined in Experiment 3, in which the task was modified so that both cues and probes were faces (to reduce distinctiveness), and also included emotional cue faces to examine the effect of more salient cues.

However, the observed changes may not be unique to faces. In the face-letter AX-CPT, participants were likely engaged in more distinctive processing of the cue because they needed to make a two-choice semantic judgement about the face (i.e., male or female?) to determine the validity of the cue. This may have helped participants - older adults in particular - to distinguish valid and invalid cues and selectively use the perceptually salient and distinctive cues to guide their subsequent response to the letter probe. To address this possibility, Experiment 3 used faces as stimuli but in a paradigm that was more analogous to the letter AX-CPT by defining one of multiple faces as a valid cue (to increase processing complexity of cues) and using perceptually similar stimuli (i.e., faces) as probes (to reduce cue-probe distinctiveness). 
In summary, although it is unclear what specific elements or mechanisms caused the shift towards proactive control, it is possible that what they all have in common is the effect of reducing task demands. The modifications to the AX-CPT were intended to reduce the cognitive demands of the task and this was successfully achieved, as supported by a reduction in target error rates in the face-letter AX-CPT compared to the letter AX-CPT, as well as a reduction in overall error rates, $p<.001$. This provides support for predictions from the DMC model that older adults' proactive control deficit may be related to age-related limitations on cognitive resources which hinders the spontaneous engagement of proactive control (Braver et al., 2007). When cognitive resources are not as taxed from reduced task demands, older adults can rely less on reactive control. However, it should be emphasized that this facilitative effect was limited to RTs, with no evident changes to error rates. It may be that this effect is rather subtle and potentially only detectable with more sensitive behavioural measures, such as speed of responding or neuroimaging techniques. Additional research examining why there are different performance patterns for accuracy and RTs will be necessary in order to elucidate the manner in which particular factors affect proactive and reactive control. 


\section{Experiment 2B: Age Differences in Face-Letter AX-CPT under Negative and Positive}

\section{Mood}

Participants in Experiment 2B received a negative or positive mood induction prior to completing the face-letter AX-CPT task with faces as cues and letters as probes.

\section{Method}

\section{Participants}

Participants were 68 healthy younger adults (ages 18-29) and 55 healthy older adults (ages 65-89). Twelve younger adults and one older adult were excluded due to high DASS scores that were in the range categorized as “extremely severe”. Three younger adults were excluded due to current diagnoses of depression or anxiety; one older adult was excluded due to technical malfunctions, and one older adult was excluded due to an SBT score greater than the cut-off score of 6 . The final sample included in the analyses were 53 younger and 52 older adults. Participants were randomly assigned to undergo either the negative or positive mood inductions, resulting in four different age by emotion groups (Table 6). 
Table 6

Participant Demographic Characteristics and Cognitive Performance by Age and Mood Induction Group

\begin{tabular}{lllllllll}
\hline & \multicolumn{3}{c}{ Younger Adults } & \multicolumn{5}{c}{ Older Adults } \\
\cline { 2 - 5 } Characteristic & $\begin{array}{l}\text { Negative Mood } \\
(n=26)\end{array}$ & \multicolumn{3}{c}{$\begin{array}{l}\text { Positive Mood } \\
(n=27)\end{array}$} & $\begin{array}{l}\text { Negative Mood } \\
(n=27)\end{array}$ & \multicolumn{2}{c}{$\begin{array}{l}\text { Positive Mood } \\
(n=25)\end{array}$} \\
\hline Age & 20.62 & $(2.73)$ & 21.04 & $(3.20)$ & 72.00 & $(4.90)$ & 73.44 & $(7.33)$ \\
F/M (ratio) & $20 / 6$ & & $23 / 4$ & & $24 / 3$ & & $21 / 4$ \\
Years of edu. & 14.00 & $(2.18)$ & 14.41 & $(1.59)$ & 14.85 & $(2.33)$ & 15.28 & $(3.09)$ \\
Health rating & 7.85 & $(1.05)$ & 7.65 & $(1.28)$ & 8.33 & $(1.41)$ & 8.92 & $(0.93)$ \\
FTP & 50.65 & $(7.57)$ & 51.89 & $(9.61)$ & 38.56 & $(11.15)$ & 40.48 & $(12.03)$ \\
PANAS-PA & 24.08 & $(8.05)$ & 26.00 & $(7.64)$ & 35.67 & $(6.09)$ & 38.92 & $(5.85)$ \\
PANAS-NA & 14.96 & $(3.45)$ & 12.15 & $(3.34)$ & 13.63 & $(5.81)$ & 12.04 & $(3.30)$ \\
DASS-Dep & 8.38 & $(6.50)$ & 7.26 & $(5.44)$ & 5.19 & $(5.69)$ & 4.64 & $(4.99)$ \\
DASS-Anx & 7.08 & $(4.57)$ & 6.81 & $(5.58)$ & 3.56 & $(4.24)$ & 2.75 & $(2.88)$ \\
Corsi & 0.61 & $(0.15)$ & 0.58 & $(0.29)$ & 0.27 & $(0.15)$ & 0.29 & $(0.21)$ \\
Patt. Compar. & 67.81 & $(47.44)$ & 71.19 & $(11.29)$ & 47.44 & $(9.92)$ & 47.74 & $(9.62)$ \\
DCCST & 9.04 & $(0.64)$ & 9.06 & $(0.55)$ & 7.59 & $(1.17)$ & 7.73 & $(1.14)$ \\
Flanker & 9.42 & $(0.53)$ & 9.50 & $(0.44)$ & 8.50 & $(0.81)$ & 8.34 & $(0.82)$ \\
Vocabulary & $1497.46(211.78)$ & 1551.15 & $(139.67)$ & 1930.37 & $(226.87)$ & $2082.00(612.66)$ \\
SBT & & & & & 0.74 & $(1.13)$ & 0.96 & $(1.65)$ \\
\hline
\end{tabular}

Note. Mean scores with standard deviations presented in parentheses. F/M (ratio) = female/male ratio; years of edu. = average number of years of formal education; health rating = report of self-rated health on a scale from 1 (poor) to 10 (excellent); FTP = Future Time Perspective Scale; PANAS-PA/NA = Positive Affect Negative Affect Schedule; DASS-Dep/Anx = Depression, Anxiety, and Stress Scales; Corsi $=$ Corsi Block; Pattern compar. $=$ Pattern Comparison Processing Speed Test; DCCST = Dimensional Change Card Sort Test; Flanker = Flanker Inhibitory Control and Attention Test; Vocabulary = Picture Vocabulary Test; SBT = Short Blessed Test ${ }^{*} p<.05 .{ }^{* *} p<.001 .{ }^{x}$ denotes the use of chi-square statistic; all other analyses used independent $t$-tests $d=$ Cohen's $d$ effect size calculation for between subjects

\section{Mood Inductions}

Participants were randomly assigned to the negative or positive mood induction group. 


\section{Face-Letter AX-CPT Task}

The same face-letter AX-CPT task was used as in Experiment 2A. The task procedure was also the same.

\section{Procedure}

The procedure was identical to that of Experiment 2A except that participants received either a negative or positive mood induction.

\section{Results}

\section{Mood Ratings and Induction Questionnaire}

Valence. The valence ratings were submitted to a 2 (Age: younger vs. older) $\times 2$ (Mood induction: negative, positive) $\times 6$ (Time point) mixed ANOVA. The means are presented in Figure 11 (middle and right panels). There was a main effect of time point, $F(3.22,325.01)=$ $30.93, p<.001, \eta_{\mathrm{p}}{ }^{2}=.23$. There were also main effects of age, $F(1,101)=7.17, p=.009, \eta_{\mathrm{p}}{ }^{2}=$ .07 , and mood induction, $F(1,101)=61.18, p<.001, \eta_{\mathrm{p}}{ }^{2}=.38$. The two- and three-way interactions were also significant, $F \mathrm{~s}>5.13, p \mathrm{~s}<.001, \eta_{\mathrm{p}}{ }^{2} \mathrm{~s}>.05$. To clarify these interactions, separate 2 (Age) $\times 6$ (Time point) mixed ANOVAs were conducted for each mood induction group. These analyses revealed that the main effect of time point was significant in both induction groups, $F \mathrm{~s}>17.00, \mathrm{ps}<.001$. In the positive induction group, planned contrasts with baseline ratings revealed that ratings were higher after the first induction, equivalent after the second induction, and lower at all other time points. Ratings were also higher after the first and second induction compared to all other time points, $p s<.001$, which did not differ from each other, $p s>$.642. The age and age by time point effects were not significant.

For the negative induction group, the main effects of time point, age, and the age by time point interaction were significant, $p \mathrm{~s}<.029$. To further examine this, paired samples $t$-tests were 
conducted for each age group separately. For younger adults, all comparisons with baseline were significant, with lower ratings at all subsequent time points, $p \mathrm{~s}<.001$. Ratings at post-induction did not differ from all subsequent time points, $p s>.044$, except for higher ratings at the second pre-induction time point, $p=.005$. Ratings dropped again after the second post induction and did not change after the second AXCPT block, $p=.327$. Similar to younger adults, older adults had lower ratings at all subsequent time points compared to baseline, $p s<.001$. Ratings did not differ between both post-induction time points, $p=.847$, and ratings at both were lower than at other time points, $p s<.001$. Ratings after the first AXCPT block and at the second pre-induction time points did not differ, $p=.691$. Ratings after the second AXCPT block were lower than after the first block, $p=.006$, and at the second pre-induction time point, $p=.005$. Additionally, age group comparisons revealed that younger and older adults did not differ at baseline or after the two induction time points, ps > .306; however, younger adults reported lower ratings at all other time points, $p s<.004$.

Finally, independent samples $t$-tests confirmed that ratings in both mood groups did not differ at baseline, $p=.863$, and were significantly higher in the positive than negative induction groups at all other time points, $p s<.001$. When these analyses were repeated separately for each age group, the pattern of results remained the same for younger adults. For older adults, the positive and negative induction groups only differed at the two post-induction time points and after the second AXCPT block, ps <.024, with higher ratings in the positive than negative group.

Arousal. The analyses on SAM arousal ratings revealed main effects of age, $F(1,101)=$ 27.87, $p<.001, \eta_{\mathrm{p}}{ }^{2}=.22$, and mood induction, $F(1,101)=8.00, p=.006, \eta_{\mathrm{p}}{ }^{2}=.07$ (Figure 12, middle and right panels). These effects were qualified by an age by mood interaction, $F(1,101)$ $=5.95, p=.016, \eta_{\mathrm{p}}{ }^{2}=.06$, driven by lower arousal ratings in the positive than negative induction 
group for younger adults, $t(51)=-4.29, p<.001$, but no difference between groups for older adults, $t(50)=-0.25, p=.808$. There was also a main effect of time point, $F(3.54,357.45)=$ 9.38, $p<.001, \eta_{\mathrm{p}}{ }^{2}=.09$, which was qualified by an age by time point interaction, $F(5,505)=$ 2.92, $p=.013, \eta_{\mathrm{p}}{ }^{2}=.03$. For younger adults, all planned contrasts revealed higher arousal ratings at baseline than subsequent time points. All other comparisons were not significant, $p \mathrm{~s}>.071$. All comparisons for older adults were not significant, $p s>.082$. Finally, independent samples $t$ tests revealed significant age differences at every time point, $p$ s $<.003$, except at baseline, $p=$ .113, with younger adults reporting lower arousal ratings than older adults (Figure 12, middle and right panels).

Induction questionnaire. Separate 2 (Age: younger, older) $\times 2$ (Mood induction: negative, positive) two-way ANOVAs were conducted to examine group differences on the questionnaire responses. These revealed several group differences. Those in the positive induction group $(M=2.19, S D=1.07)$ reported being more familiar with the music they heard than those in the negative induction group $(M=1.62, S D=0.86), F(1,104)=11.30, p=.001$, $\eta_{\mathrm{p}}{ }^{2}=.10$. Older adults also reported being more familiar with both the music they heard ( $M_{\text {younger }}$ $\left.=1.57, S D_{\text {younger }}=0.80 ; M_{\text {older }}=2.25, S D_{\text {older }}=1.08\right), F(1,104)=15.94, p<.001, \eta_{\mathrm{p}}{ }^{2}=.14$, and with classical music $\left(M_{\text {younger }}=2.53, S D_{\text {younger }}=0.72 ; M_{\text {older }}=3.08, S D_{\text {older }}=0.74\right), F(1,104)=$ $15.03, p<.001, \eta_{\mathrm{p}}{ }^{2}=.13$, than younger adults. Older adults $(M=7.04, S D=2.14)$ also reported higher ratings of induction effectiveness than younger adults $(M=5.66, S D=2.43)$. For these questions, the age by mood induction interactions did not reach significance, $F \mathrm{~s}<1.80$, ps $>$ .183. For the question on slideshow processing, $92.38 \%$ indicated that they were paying attention, $2.86 \%$ indicated they were trying to memorize, and $4.7 \%$ indicated they were doing 
neither. There were no age or mood induction differences for any of the categories, $\chi^{2} s<0.58$, $p s$ $>.448$.

Overall, both age groups reported higher valence ratings after the positive mood inductions. Ratings were lower after the AX-CPT blocks but average ratings remained above 6 . Both age groups also reported lower valence ratings after the negative mood inductions (average ratings $<4$ ) but younger adults stayed in these negative moods longer, whereas older adults returned to more positive moods following the AX-CPT blocks. Importantly, there were no age group differences at the critical baseline and post-induction time points. Younger adults in the positive induction group also reported lower arousal ratings as compared to the negative induction.

\section{AX-CPT Performance}

Only response time data for correct responses were analyzed (95.95\% of all trials). There were no correct response time data for probe-lure trials for one younger (positive mood condition) and one older adult (negative mood condition) as they did not make any correct responses on these trials; thus these data points were treated as missing values.

\section{Target trials.}

Error rates. A 2 (Age: younger, older) $\times 2$ (Mood induction: negative, positive) two-way ANOVA on error rates did not reveal any significant effects or interactions, $F$ s $<0.38$, $p s>.538$ (Figure 13, middle and right panels).

Response times. Similar to errors, the same ANOVA on RTs did not reveal any significant effects or interactions, $F s<2.52$, ps $>.116$ (Figure 14, middle and right panels). 


\section{Non-target trials.}

Error rates. Error rates were submitted to a 2 (Age: younger, older) $\times 2$ (Mood induction: negative, positive) $\times 3$ (Trial type: control, probe-lure, cue-lure) mixed ANOVA. There was a main effect of trial type, $F(1.32,132.78)=23.18, p<.001, \eta_{\mathrm{p}}{ }^{2}=.18$. Error rates were lower for control than probe- and cue-lure trials, and were higher for probe- than cue-lure trials, ps $<.008$. All other effects were not significant, $p s>.429$. Although the three-way interaction was not significant, planned comparisons were conducted to examine specific hypotheses regarding differences between mood groups in age-related reactive and proactive patterns. First, paired samples $t$-tests were conducted for each age by mood induction group separately. Younger adults in both induction groups performed similarly, with fewer errors for control than probe- and cuelure trials, $p s<.007$; there were no differences between the latter, $p s>.218$. Similarly, older adults in both induction groups demonstrated the same patterns, with more errors on probe-lure than control trials, $p s<.005$, and more probe- than cue-lure errors, $p s<.05$. Older adults in the negative induction group made fewer errors on control than cue-lure trials, $p<.001$, but there was no such difference in the positive induction group, $p=.134$. The three-way interaction was not significant so these findings should be interpreted with caution. Finally, independent $t$-tests were conducted to examine age or mood induction differences for each trial type. For both induction groups, younger and older adults did not differ on any of the trial types, $p s>.177$. Additionally, there were no mood induction differences on any of the trial types for either younger or older adults, ps $>.443$ (Figure 13, middle and right panels).

Response times. The same mixed ANOVA was used to analyze the RT data. There was a main effect of age, $F(1,99)=4.22, p=.043, \eta_{\mathrm{p}}{ }^{2}=.04$, with faster RTs for younger than older adults. There was also a main effect of trial type, $F(1.59,157.60)=330.07, p<.001, \eta_{\mathrm{p}}{ }^{2}=.77$, 
with faster responses to probe-lure than cue-lure trials, which were in turn responded faster to than control trials, $p s<.002$. Although the mood induction by trial type interaction was trending significance, $p=.084$, all other interactions were not significant, $p$ s $>.108$. Nevertheless, the RT data were submitted to the same planned comparisons as the error data to examine performance differences in each age by mood induction group. For all groups, paired samples $t$-tests revealed similar trial type differences as seen in the mixed ANOVA, with faster responses to control and probe-lure than cue-lure trials, $p s<.001$. However, the finding of faster responses to probe-lure than control trials was seen only for the younger adults in the negative induction group, $p=.011$. This difference was not significant in any of the other groups, $p s>.130$.

Additionally, independent $t$-tests were conducted to examine age or mood group differences for each trial type. For both induction groups, no age differences were found for control or cue-lure trials, $p s>.10$. For probe-lure trials, no age differences were seen in the positive induction groups, $p=.539$, but responses were faster in younger than older adults in the negative induction groups, $p=.041$. Finally, independent $t$-tests comparing mood induction groups for each age group separately revealed no differences for control or probe-lure trials. For cue-lure trials, there were no mood induction group differences in younger adults. However, older adults had slower responses in the negative than positive induction group, $p=.040$ (Figure 12, middle and right panels).

Proactive index. One sample $t$-tests for each age by mood induction group were conducted to examine whether index values differed significantly from zero. For younger adults in both groups, the proactive index for errors was not significant, $p \mathrm{~s}>.529$, but the index for RTs was significantly greater than zero, $p s<.001$. For older adults, the proactive index for errors was significantly less than zero in the positive induction group, $p=.049$, and this difference was 
marginally significant in the negative induction group, $p=.057$. Similar to younger adults, the proactive index for RTs was also significantly greater than zero, ps $<.001$.

To examine group differences in index values, separate one-way ANOVAs on errors and RTs were conducted, with age and induction group as between-subjects factors. The proactive index for errors was significantly smaller in older than in younger adults, $F(1,104)=6.87, p=$

$.010, \eta_{\mathrm{p}}{ }^{2}=.06$; however, the age groups did not differ in proactive indices for RTs, $p \mathrm{~s}>.169$. No other effects were significant, $p s>.900$. The means for the proactive indices for errors and RTs are presented in the middle and right panels of Figure 15 and Figure 16, respectively.

\section{Individual Differences Measures}

Separate 2 (Age: younger, older) $\times 2$ (Mood induction: negative, positive) univariate ANOVAs for each measure revealed various group differences. Correlation analyses were conducted with measures that showed significant age differences and the proactive index for errors, which also showed a significant age difference (larger proactive index for younger than older adults). The following measures correlated with the error index: FTP, $r(105)=.24, p=$ .012 ; Corsi block, $r(104)=.23, p=.018$; DCCST, $r(101)=.22, p=.030$, and Flanker, $r(101)=$ $.22, p=.025$. Including these variables in the ANCOVA on proactive index for errors once again revealed that the effect of age was no longer significant, $p=.850$; all other effects were also not significant, Fs $<.963$, ps $>.329$.

\section{Cross-Experiment Analyses}

Experiment 2A vs. 2B. The first set of cross-experiment analyses compared the effects of the different mood inductions on the face-letter AX-CPT task. These analyses compared Experiment 2A (neutral induction + face-letter AX-CPT) with the negative and positive induction groups of Experiment 2B. 
Mood analyses. Analyses focused on comparing ratings in the neutral versus negative and positive induction groups, across the various time points. Ratings were higher in the positive than neutral group at both post-induction time points $\left(M \mathrm{~s}_{\text {pos }}>7.02, S D \mathrm{~s}_{\text {pos }}<1.49 ; M \mathrm{~s}_{\text {neut }}<6.87\right.$, $\left.S D \mathrm{~s}_{\text {neut }}<1.83\right), p s<.009$, and approached significance at the second pre-induction time point $\left(M_{\text {pos }}=6.52, S D_{\text {pos }}=1.71 ; M_{\text {neut }}=5.85, S D_{\text {neut }}=1.79\right), p=.052$. Ratings were lower in the negative than neutral group after both inductions $\left(M \mathrm{~s}_{\text {neg }}<3.77, S D \mathrm{~s}_{\text {neg }}<1.78 ; M \mathrm{~s}_{\text {neut }}<6.87\right.$, $\left.S D s_{\text {neut }}<1.83\right)$, ps $<.001$, and after both AXCPT blocks $\left(M s_{\text {neg }}<5.47, S D s_{\text {neg }}<1.85 ; M s_{\text {neut }}<\right.$ $\left.6.13, S D s_{\text {neut }}<1.96\right), p s<.050$. These results suggest that the positive and negative inductions were effective at inducing the intended moods, relative to the neutral induction.

The arousal ratings for the negative and neutral induction groups did not differ at any time points. For the positive group, arousal ratings were lower than those of the neutral group at the earlier time points (baseline and the first post-induction), $p s<.020$, and were approaching significance at the middle time points (post-block 1 and pre-induction 2), ps <.059.

\section{AX-CPT performance.}

Target trials. A 2 (Age: younger, older) $\times 3$ (Mood induction: neutral, negative, positive) two-way ANOVA was conducted. For errors, the age by mood interaction was significant, $F(2$, $156)=3.47, p=.034, \eta_{\mathrm{p}}{ }^{2}=.04$, and all main effects were not significant, $F \mathrm{~s}<1.33$, $p \mathrm{~s}>.250$. To clarify this interaction, separate one-way ANOVAs were conducted for each age group, with mood as a between-subjects factor. There was a main effect of mood for younger adults, $F(2,79)$ $=4.34, p=.016, \eta_{\mathrm{p}}{ }^{2}=.10$, but this was not significant in older adults, $p=.743$. Younger adults in the positive $(M=0.02, S D=0.03)$ and negative $(M=0.02, S D=0.02)$ induction groups made fewer errors than those in the neutral group $(M=0.05, S D=0.05), p s<.040$. However, these differences were not significant after Bonferroni adjustments for multiple comparisons. The 
same ANOVA was conducted on the RT data. No main effects or interactions were significant, $F s<1.43, p s>.244$

Non-target trials. A 2 (Age: younger, older) $\times 3$ (Mood induction: neutral, negative, positive) $\times 3$ (Trial type) mixed ANOVA did not reveal a main effect or interaction of mood induction. However, the RT analysis revealed a mood induction by trial type interaction, F(4, 294) $=2.49, p=.043, \eta_{\mathrm{p}}{ }^{2}=.03$. To elucidate this interaction, separate univariate ANOVAs were conducted for each trial type separately, with mood induction as a between-subjects factor. These analyses revealed a significant main effect of mood induction for cue-lure trials only, $F(2,156)=$ 3.36, $p=.037, \eta_{\mathrm{p}}{ }^{2}=.04$; this effect was not significant for the other trial types, $F \mathrm{~s}<1.24, \mathrm{ps}>$ .292. Post-hoc comparisons on cue-lure trials indicated that the positive induction group $(M=$ $0.69, S D=0.37)$ did not differ from the neutral induction group $(M=0.67, S D=0.40), p=.855$. Both the positive and neutral groups differed from the negative group $(M=0.89, S D=0.55)$, with slower cue-lure responses in the latter, $p s<.042$. Based on visual inspection, it appeared that this effect was mainly driven by older adults’ performance. The same comparisons conducted for each age group separately revealed no significant effects in younger adults; however, for older adults, cue-lure responses were slower in the negative than positive group, $p=$ .040 , and were slightly slower than the neutral group (approaching significance), $p=.069$. However, these results should be interpreted with caution, as these effects were not significant after conservative Bonferroni corrections.

Proactive index. The 2 (Age: younger, older) × 3 (Mood induction: neutral, negative, positive) two-way ANOVA on the proactive index for errors did not reveal a main effect or interaction of mood induction, $p \mathrm{~s}>.326$. This was true for the proactive index for RTs as well, ps $>.100$. 
Experiment 1B vs. 2B. The second set of cross-experiment analyses compared the effects of the different AX-CPT task versions when participants were induced into negative moods. These analyses compared Experiment 1B (negative mood induction + standard letter AX-CPT) with the negative mood group of Experiment 2B (negative mood induction + faceletter AX-CPT).

Mood ratings. Independent samples t-tests were used to check whether the negative mood induction resulted in similar mood ratings across the two experiments. Despite similar ratings across experiments at most time points (including baseline), $p \mathrm{~s}>.084$, ratings at both post-induction time points were lower in Experiment 2B than 1B, $p s<.009$. The same set of $t$ tests on arousal ratings did not reveal any significant effects, $p s>.287$. Thus, it appears the negative induction was more effective at lowering moods (at post-induction time points) in Experiment 2B than 1B.

\section{AX-CPT performance.}

Target trials. A 2 (Age: younger, older) × 2 (Version: letter, face-letter) two-way ANOVA revealed a main effect of version, $F(1,107)=11.52, p=.001, \eta_{\mathrm{p}}{ }^{2}=.10$. Error rates were higher in the standard letter than the face-letter AX-CPT. All other effects were not significant, $F \mathrm{~s}<0.27, p s>.608$.

Non-target trials. A 2 (Age: younger, older) $\times 2$ (Version: letter, face-letter) $\times 3$ (Trial type: control, probe-lure, cue-lure) mixed ANOVA was conducted on the error rates and RTs. For errors, the only significant result was a main effect of trial type, $F(1.21,126.22)=26.47, p<$ $.001, \eta_{\mathrm{p}}{ }^{2}=.20$. All trial types differed from each other, with the lowest error rates for control trials, followed by cue-lure, then probe-lure trials, $p s<.005$. All other effects were not significant, $F \mathrm{~s}<.2 .16, p \mathrm{~s}>.118$. 
For RTs, there were main effects of age, version, and trial type, $F \mathrm{~s}>7.72, p s<.006, \eta_{\mathrm{p}}{ }^{2} \mathrm{~s}$ $>.07$, as well as a version by trial type interaction, $F(2,206)=51.18, p<.001, \eta_{\mathrm{p}}{ }^{2}=.33$. The age by trial type and the 3-way interactions were approaching significance, $F \mathrm{~s}>2.81, p \mathrm{~s}<.062, \eta_{\mathrm{p}}{ }^{2} \mathrm{~s}$ $>$.03. The age by version interaction was not significant, $p=.660$. To elucidate the significant interactions, the RT proactive indices were submitted to 2 (Age) $\times 2$ (Version) two-way ANOVAs. This revealed a main effect of version, $F(1,106)=76.79, p<.001, \eta_{\mathrm{p}}{ }^{2}=.43$. Proactive indices were larger in the face-letter $(M=0.24, S D=0.12)$ than the letter $(M=0.02$, $S D=0.13)$ version. The main effect of age group was trending significance, $F(1,106)=3.30, p$ $=.072, \eta_{\mathrm{p}}{ }^{2}=.03$, with slightly greater proactive index values in younger than older adults. The age by version interaction was not significant, $p=.148$.

Together, these results mirror the patterns seen in the prior cross-experiment analysis comparing the two neutral induction groups with different versions of the AX-CPT. It appears the version facilitated engagement in proactive control as indexed by fast probe-lure but slow cue-lure responses. This resulted in a proactive index for RTs that was significantly larger in the face-letter than letter version. However, this pattern did not appear to be modulated by negative induction as results were comparable to those observed under neutral induction.

\section{Discussion}

In Experiment 2B, participants completed the face-letter AX-CPT and underwent a positive or negative mood induction. The results again showed that the face-letter AX-CPT resulted in enhanced proactive control reflected in the RT data. However, there were only minimal effects of mood on cognitive control patterns in younger and older adults.

Counter to the hypothesis that negative mood may not have any effects for either age group (H 10), there was some evidence of effects due to negative relative to positive induced 
moods. Specifically, there was some evidence of slower cue-lure RTs in older adults under negative compared to the positive induction. This was not expected as it was hypothesized that positive mood may enhance proactive control in older adults ( $\mathrm{H} 11)$. There are some speculative explanations for this effect. For example, studies have found that older adults induced into negative moods seek out positive stimuli, hypothesized to be driven by motivations to regulate out of negative moods (Isaacowitz et al., 2008). Additionally, there is research indicating that older adults may naturally perceive more positivity in faces displaying ambiguous expressions (Kellough \& Knight, 2011). Combining these two findings, a speculative explanation may be that older adults induced into negative, relative to positive or neutral moods, had enhanced cue processing because they were motivated to regulate out of negative moods and thus sought out items that could help with mood repair, such as the face cues. Despite the fact that the face cues were displaying neutral expressions, it may have been possible that those induced into negative moods perceived positivity in these neutral faces and were motivated to attend to them to help with mood repair. Although this is a speculative explanation, it may partially explain why results of Experiment 1B (with only letter stimuli) did not show similar enhanced cue processing in older adults under negative condition. Future research could examine whether older adults in negative moods show enhanced processing of face cues when they display positive versus negative and neutral expressions. It should be emphasized that although the negative induction was identical in both experiments, the post-induction ratings were lower in the current experiment compared to Experiment 1B. It is unclear why this occurred, as baseline ratings were comparable in both experiments. The sample characteristics and other mood measures (e.g., PANAS) were also similar in both groups. Additionally, the rating of induction effectiveness did 
not differ. Nevertheless, this hinders the ability to make conclusions regarding the effects of the negative induction in Experiment 1B.

Although it was hypothesized that cognitive control in younger adults would not be affected by negative induced moods ( $\mathrm{H}$ 10), it was hypothesized that they would be affected by positive moods ( $\mathrm{H}$ 11). However, the results lacked evidence suggesting reduced proactive control in younger adults. This is inconsistent with results from various studies that found positive moods and stimuli reduced proactive control in younger adults (e.g., Dreisbach, 2006; Fröber \& Dreisbach, 2012). It may have been possible that the positive induction was too mild for observable effects to emerge. Previous studies presented participants with positive versus neutral stimuli on every trial (e.g., Dreisbach, 2006). However, other studies with similar paradigms found enhanced proactive control in younger adults (Chiew \& Braver, 2014). Thus, it may be possible that the effects of induced positive moods on proactive control are not very consistent.

Cross-experiment analyses were also conducted to compare proactive and reactive control in Experiment 1B versus the negative induction groups in Experiment 2B to examine whether emotion effects (i.e., of negative inductions) would be more evident on a less demanding version of the AX-CPT. Results indicated a shift away from reactive and toward proactive control in Experiment 2B in RTs, driven by the change from letter to face cues. The shift away from reactive control was evident in both age groups, but the shift toward proactive control was evident only for older adults. These findings are consistent with the results from analyses that compared these versions but with neutral mood inductions (reported in Experiment 2A). As such, this finding of greater proactive control appears to be driven primarily from the version change, with minimal or no changes arising due to the negative mood inductions. This 
goes against the hypothesis that mood effects would be more evident on an easier versus more difficult task. However, this conclusion is limited to negative moods, as positive inductions were paired with the face-letter AX-CPT only. However, the positive induction did not appear to significantly change control patterns in either age group so it may be possible that effects due to induced positive moods may not be observable with either AX-CPT version. 


\section{Chapter 6: Experiment 3}

\section{Effect of Neutral and Emotional Faces in Face AX-CPT Task}

Experiment 3 examined the effect of emotional face cues on cognitive control in older adults. Participants completed a modified AX-CPT task that consisted of neutral and emotional faces as cue and probe stimuli, hereafter referred to as the face AX-CPT.

\section{Objectives and Rationale}

Emotional stimuli in tasks measuring components of cognitive control (e.g., working memory; Borg et al., 2011; Truong \& Yang, 2014) reveal different effects of positive and negative stimuli on performance in younger and older adults. An unaddressed question is whether emotional stimuli can influence proactive and reactive control patterns in older adults. Experiment 3 addressed this question. Specifically, older adults completed the face AX-CPT that used face stimuli for both cues and probes. The emotional expression (happy, sad, or neutral) of the valid face cue was manipulated across blocks. This manipulation tested whether cue processing would differ depending on the emotion expressed by the valid cue face.

Additionally, Experiment 2 revealed proactive control in younger and older adults using the face-letter AX-CPT. Analyses comparing performance on the letter versus face-letter AXCPT revealed greater proactive control in the latter. However, the mechanisms driving this change are not clear. It was not clear whether it was due to: a) the inclusion of faces as stimuli, b) the distinction between face cues and letter probes, or c) reduced number of competitor choices. Thus, the face AX-CPT used in Experiment 3 was designed to be as similar as possible to the parameters of the standard letter AX-CPT (used in Experiment 1), except for the use of face instead of letter stimuli. Specifically, the number of competitor stimuli were similar/equated. A comparable task rule was selected to be similar to that of A versus non-A (i.e., face A versus 
non-face A). Thus, Experiment 3 also served to help clarify the potential mechanism underlying the finding of enhanced proactive control in older adults found in Experiment 2.

\section{Hypotheses}

$\boldsymbol{H}$ 11: It was hypothesized that greater proactive control would be found in the positive versus negative and neutral block. This hypothesis was based on findings of a general positivity effect in older adults' processing preferences (Reed et al., 2014), as well as findings that older adults perform well at maintaining positive stimuli and affect in working memory (Mikels et al., 2005; Truong \& Yang, 2014). Specifically, it was hypothesized that older adults would engage in greater cue processing and maintenance when the cues were happy faces in the positive block. This would be reflected as poorer cue-lure performance in the positive relative to the negative and neutral blocks. Additionally, based on findings that older adults experience greater interference and poorer inhibitory control for negative than positive and neutral stimuli, it was hypothesized that differences would be greatest when comparing performance for negative versus positive face cues. However, there was no prediction as to whether it would specifically impair proactive or reactive control.

\section{Method}

Older adults completed the face AX-CPT with faces as cue and probes. The emotional valence (positive, negative, neutral) of the emotional expression displayed by face cues was manipulated across three blocks.

\section{Participants}

Thirty-three healthy older adults (ages 65-90, $M=73.36, S D=7.10$ ) participated in Experiment 3. One participant was excluded due to a SBT score of six. Two participants did not 
have useable data due to technical issues with the E-Prime software. The final sample consisted of 30 older adults (Table 7).

Table 7

Participant Demographic Characteristics and Cognitive Performance

\begin{tabular}{lll}
\hline Characteristic & \multicolumn{2}{l}{ Older Adults } \\
\cline { 2 - 3 } & $M$ & $(\mathrm{n}=30)$ \\
\hline Age & 73.30 & $(7.14)$ \\
Female/male (ratio) & $18 / 12$ & \\
Years of education & 15.53 & $(3.20)$ \\
Health rating & 8.38 & $(1.32)$ \\
Digit symbol & 58.47 & $(13.96)$ \\
Digit span & 7.00 & $(1.35)$ \\
Shipley vocabulary & $34.67^{\mathrm{a}}$ & $(3.81)$ \\
FTP & 40.43 & $(12.37)$ \\
PANAS-Positive & 39.40 & $(7.24)$ \\
PANAS-Negative & 11.97 & $(2.76)$ \\
DASS-Depression & 5.17 & $(6.31)$ \\
DASS-Anxiety & 3.79 & $(4.55)$ \\
SBT & 1.20 & $(1.45)$ \\
\hline
\end{tabular}

Note. Education = average number of years of formal education; health rating = report of self-rated health on a scale from 1 (poor) to 10 (excellent); FTP = Future Time Perspective Scale; PANAS-Positive/Negative = Positive Affect Negative Affect Schedule; DASS-Depression/Anxiety = Depression, Anxiety, and Stress Scales; SBT = Short Blessed Test; ${ }^{a}$ mean score based on $n=25$ because this task was not administered for the first five participants. 


\section{Face AX-CPT Task}

Experiment 3 used a modified AX-CPT task that used female face images as cue and probe stimuli. The valid-cue and valid-probe were two specific faces. The emotional expression (happy, sad, and neutral) of the valid-cue face was manipulated across three blocks.

Stimuli. Face stimuli were obtained from the FACES collection (described in the Method section of Experiment 2A). A subset of 23 different older female faces (ages 69-80) displaying neutral, happy, and sad facial expressions were selected for the face AX-CPT ${ }^{11}$. This subset of faces was selected based on the data from the FACES database validation study (Ebner et al., 2010). This data consisted of the average rater accuracy, which was the percentage of raters who were able to correctly identify the intended emotional expression displayed by the face. This data was used to select the subset of faces used as stimuli in the face AX-CPT. First, a particular female was chosen to serve as the valid-cue face in all blocks. As the emotional expression (neutral, happy, and sad) of the valid-cue face was going to be manipulated across blocks (i.e., the same face displaying different emotional expressions in each block), it was important to select a face that received high rater identification accuracy raters across all three emotional categories. Additionally, it was important that the identification accuracy was very similar for happy and sad expressions. The database contains rating accuracies for older adult raters ( $M$ age $=73.6, S D=2.80$ ) who participated in the validation study; as such, these accuracies were used to be comparable to the older participants recruited for Experiment 3. Based on these criteria, the

\footnotetext{
${ }^{11}$ Only older female faces were selected. This decision was based on the validation data for the FACES database, which indicated lower rater accuracy for recognition of the emotional expression of older male compared to older female faces (Ebner et al., 2010). As it was hypothesized that cognitive control would vary by emotional expressions, it was important that the faces used would allow for easy recognition of the intended emotional expressions. In order to minimize the potential confound of unclear emotional expressions of the stimuli, the task was constrained to using female faces only. Older faces were selected to be as maximally self-relevant and meaningful to the older participants in this experiment.
} 
face that best met all the requirements was image code "012_o_f'” (Figure 17). This face received high older adult rater identification accuracies across all three emotions with equivalent rater accuracy scores for the happy (90.50\%) and sad image (91.00\%); the identification accuracy was high for her neutral expression as well (86.00\%). Next, a valid-probe face was selected. This face would be displaying the same neutral expression across all blocks. Thus, image code "079_o_f_n_a” was selected due to a high rater accuracy (100\%) for her neutral face image (Figure 17). The remaining 21 faces were selected based on high average ratings across the emotional categories $(M=85.62 \%, S D=5.83)$. These faces were randomly assigned to serve as invalid-cues and probes. All selected images for the face AX-CPT had received individual accuracy scores greater than $60 \%$.

Trial procedure. The trial procedure was similar in structure to the other AX-CPT tasks, with some differences in timing. The presentation time for each cue and probe was not changed (750 ms each) but the delay between them was reduced to $1500 \mathrm{~ms}$ from $5000 \mathrm{~ms}$ in the letter and face-letter AX-CPT. Previous studies did not find that a long (e.g., $5000 \mathrm{~ms}$ ) versus short (e.g., 1000 ms) delay interacted with cognitive control in older adults (e.g., Braver et al., 2005). Thus, a shorter delay was selected. The response interval for cues and probes did not change (1750 ms each) but the inter-trial interval was shortened from $1000 \mathrm{~ms}$ to $500 \mathrm{~ms}$. All timing reductions served to shorten the overall duration of a block in order to minimize fatigue effects that were likely to arise from the completion of three consecutive blocks (Chiew \& Braver, 2014). Figure 17 depicts an example target trial. 


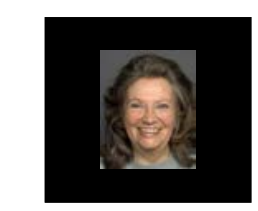

Valid Cue

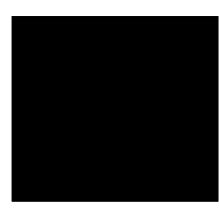

Delay

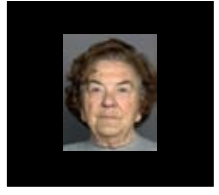

Valid Probe

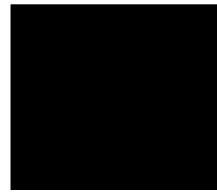

Response interval

$1000 \mathrm{~ms}$

$750 \mathrm{~ms}$

$1500 \mathrm{~ms}$

$750 \mathrm{~ms}$

Time

Figure 17. Sample illustration of a target trial in the positive block: happy face (valid cue; image code: “012_o_f”) followed by neutral face (valid probe; image code: “079_o_f”).

Trial types. The structure of the trial types was the same as the other AX-CPT tasks. The identity of the valid-cue and valid-probe faces was the same in all blocks (i.e., identity codes “012_o_f” and “079_o_f”, respectively). However, the emotional expression of the valid-cue face varied across blocks. The valid-cue face displayed a happy expression in the positive block, a sad one in the negative block, and a neutral one in the neutral block. The valid-probe face always displayed a neutral expression in all blocks. Furthermore, in the positive and negative blocks, half of the invalid-cues (used in the probe-lure and control trials) displayed the same emotion as the valid-cue and the other half displayed neutral expressions. In the neutral block, all invalid-cue faces were neutral (i.e., all faces presented in this block were neutral).

Task procedure. Participants first received instructions that introduced them to the validcue and valid-probe faces. They then began the face identification practice phase, in which they practiced identifying the valid-cue and valid-probe faces. This phase consisted of 15 self-paced trials in which participants saw one face at a time, randomly presented. Participants were asked to press the right arrow key for the valid-cue (5 trials) and the left arrow key for the valid-probe (5 trials). For all other faces, participants responded by saying “no” out loud. Following the face 
identification practice phase, participants received instructions for the face AX-CPT task and completed eight self-paced practice trials: three target trials and one trial for each of the other five trial types. This was followed by a similar practice phase of eight trials that were not selfpaced. Afterwards, participants completed the first block of 100 experimental trials. All practice phases and the experimental block were repeated another two times for the remaining two blocks, for a total of 300 trials. Participants received accuracy feedback in practice phases only and had the option to repeat practice trials as well. Block sequence was counterbalanced across participants. In one counterbalancing condition, participants began with the positive block, followed by the neutral and then negative blocks. The other counterbalancing condition started with the negative block, followed by the neutral and then positive blocks. Each block lasted approximately 7.5 minutes. The approximate total duration of the face AX-CPT task was 30 minutes.

\section{Additional Measures/Questionnaires}

Digit symbol substitution task (DSST; Wechsler, 1981). This task was used to assess visuomotor speed of processing. Participants copied a corresponding symbol into numbered boxes based on a coding legend. They were asked to copy as many items as possible until the experimenter stopped them (after 2-minutes had elapsed). The number of correct responses were summed to provide a score, ranging from 0-133; higher scores represented faster processing speeds.

Digit span task (Wechsler, 1981). This task was used to measure short-term working memory span. Participants are asked to repeat digits that are orally read to them, one digit at a time, in the sequence they were presented. Set sizes increased incrementally, from three to twelve digits, and there were two trials per set size. If participants answered the first trial in a set 
correctly, a trial from the next set size was administered. If incorrect on this first trial, participants were asked to complete the second trial. If the response to this trial was incorrect as well, the task was discontinued. The last set size answered correctly was the final span score.

Shipley vocabulary test (Shipley, 1940). This task assessed vocabulary levels. Participants were presented with a word and were asked to select a synonym word from four possible options. This test was self-paced and consisted of a total of 40 words. The final score was based on the number of correct responses.

Face AX-CPT questionnaire. This questionnaire consisted of three questions that asked participants to describe: (a) whether they noticed any differences in what they saw across the three blocks of the face AX-CPT task (e.g., the change in the emotional expressions); (b) whether they believed these differences affected their performance; and (c) whether they used any strategies to complete the task (Appendix XII). Responses to this questionnaire were designed to provide supplementary data to gain insight into how participants perceived and completed the task.

\section{Procedure}

Participants began the experiment after giving informed consent. The first task was the Digit Symbol Substitution Task (DSST). This was followed by the Digit Span task. Participants then completed the face AX-CPT task on the laptop. At the end of the task, participants were administered the Face AX-CPT questionnaire. Participants then filled out the following questionnaires, in this order: FTP, PANAS, and DASS (see General Method section for descriptions). After the questionnaires, participants completed the Shipley Vocabulary Test, followed by the Short Blessed Test. They then filled out a background information questionnaire. 
At the end of the experiment, participants received a debriefing and the cash incentive. The entire experiment was approximately 1 hour.

\section{Results}

\section{Face AX-CPT Performance}

The results for target and non-target trials were analyzed separately, for both error rates and response times (RTs). Only response time data for correct responses were used (94.48\% of all trials). In the positive block, one participant did not make any correct responses to probe-lure

trials and another participant did not respond correctly to any of the control. Two participants did not make any correct responses to probe-lure trials in the negative block. As such, the correct response time data for these trial types were missing for these three participants.

\section{Target trials.}

Error rates. A repeated measures ANOVA with Block (positive, neutral, negative) as a within-subjects factor was conducted. This revealed no significant differences across blocks, $F(1.29,37.34)=2.14, p=.148$ (Figure 18). 


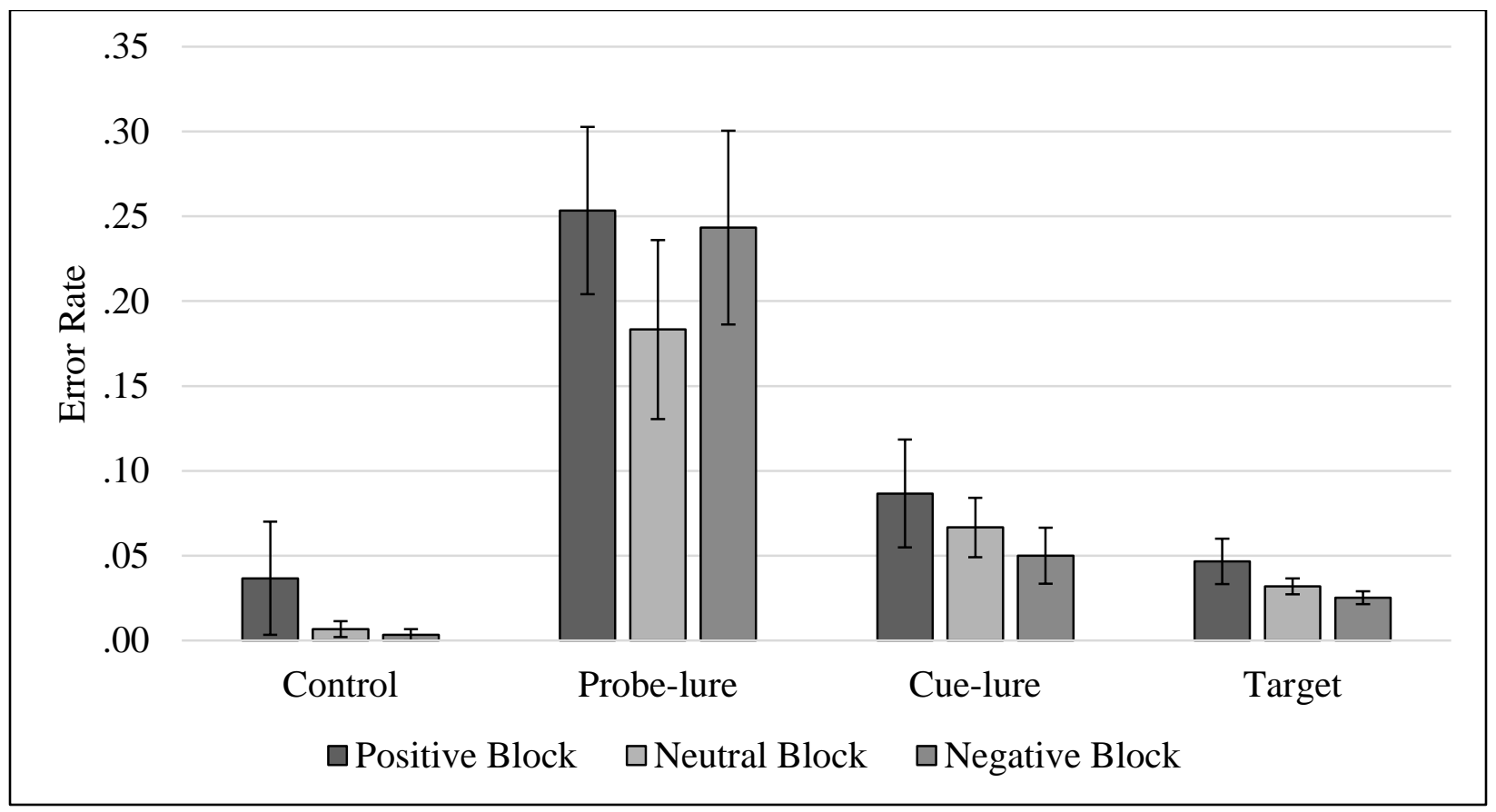

Figure 18. Mean error rates by trial type across the three blocks. Error bars denote standard error.

Response times. The same ANOVA on z-transformed RTs also revealed no significant differences across blocks, $F(1.37,39.66)=0.56, p=.574$ (Figure 19). 


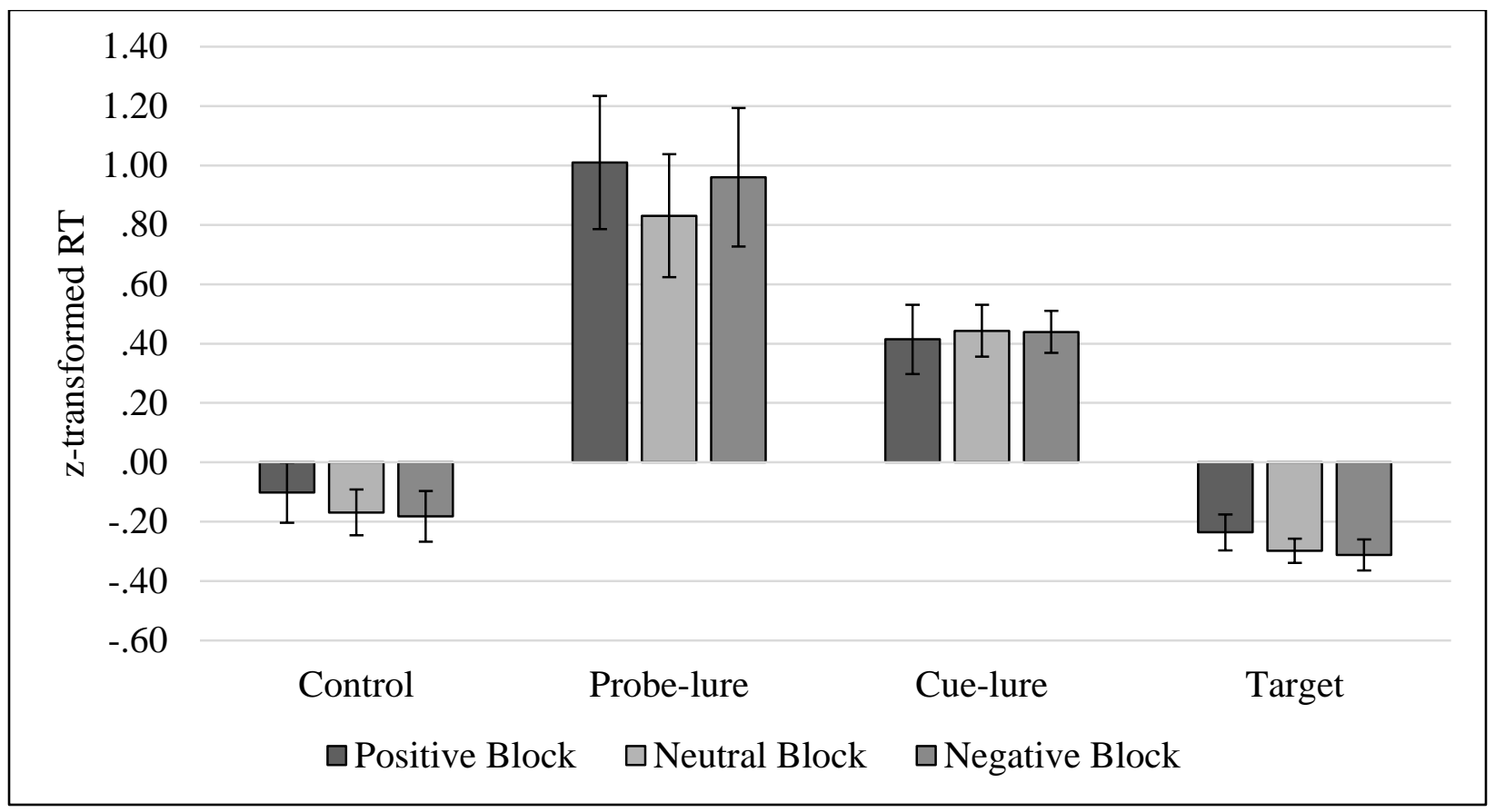

Figure 19. Mean z-transformed median RTs by trial type across the three blocks. Error bars denote standard error.

\section{Non-target trials.}

Error rates. A 3 (Block: positive, neutral, negative) $\times 3$ (Trial type: control, probe-lure, cue-lure) revealed a main effect of trial type, $F(1.08,31.33)=16.34, p<.001, \eta_{p}{ }^{2}=.36$. Planned contrasts revealed higher error rates for both probe-lure $(M=0.23, S D=0.25)$ and cue-lure $(M=$ $0.07, S D=0.08)$ than control $(M=0.02, S D=0.06)$ trials, $p s<.001$. Error rates were also higher for probe-lure than cue-lure trials, $p=.003$. There was no main effect of block or a block by trial interaction, $F \mathrm{~s}<1.10$, ps $>.317$.

Response times. The RT data were submitted to the same ANOVA as the error rates, which revealed a main effect of trial type, $F(1.35,35.05)=25.72, p<.001, \eta_{\mathrm{p}}{ }^{2}=.50$. Responses were faster for control $(M=-0.15, S D=0.37)$ than interference trials, $p s<.001$, and for cue-lure 
$(M=0.43, S D=0.40)$ than probe-lure trials $(M=0.90, S D=0.95), p=.027$. There was no main effect of block or a block by trial type interaction, Fs $<0.62$, $p s>.543$.

Proactive index. Proactive indices were calculated for errors and RTs for each block separately. These were submitted to $t$-tests to examine whether index values were significantly different from zero. These revealed indices significantly less than zero (i.e., more reactive tendencies) for errors in the emotional blocks only, ts $<2.65$, $p$ s $<.013$. All other indices did not differ from zero, $p s>.075$. The proactive indices for errors and RTs were also submitted to separate repeated measures ANOVAs with block as a within-subjects factor. For errors, the main effect of block did not reach statistical significance, $F(2,58)=2.51, p=.09, \eta_{\mathrm{p}}{ }^{2}=.08$, although there was a numerical difference between the neutral and emotional blocks, with a higher proactive index in the former (Figure 20, left panel). For RTs, there was no main effect of block, $p=.504$, suggesting a similar lack of proactive tendencies across all blocks (Figure 20, right panel). 


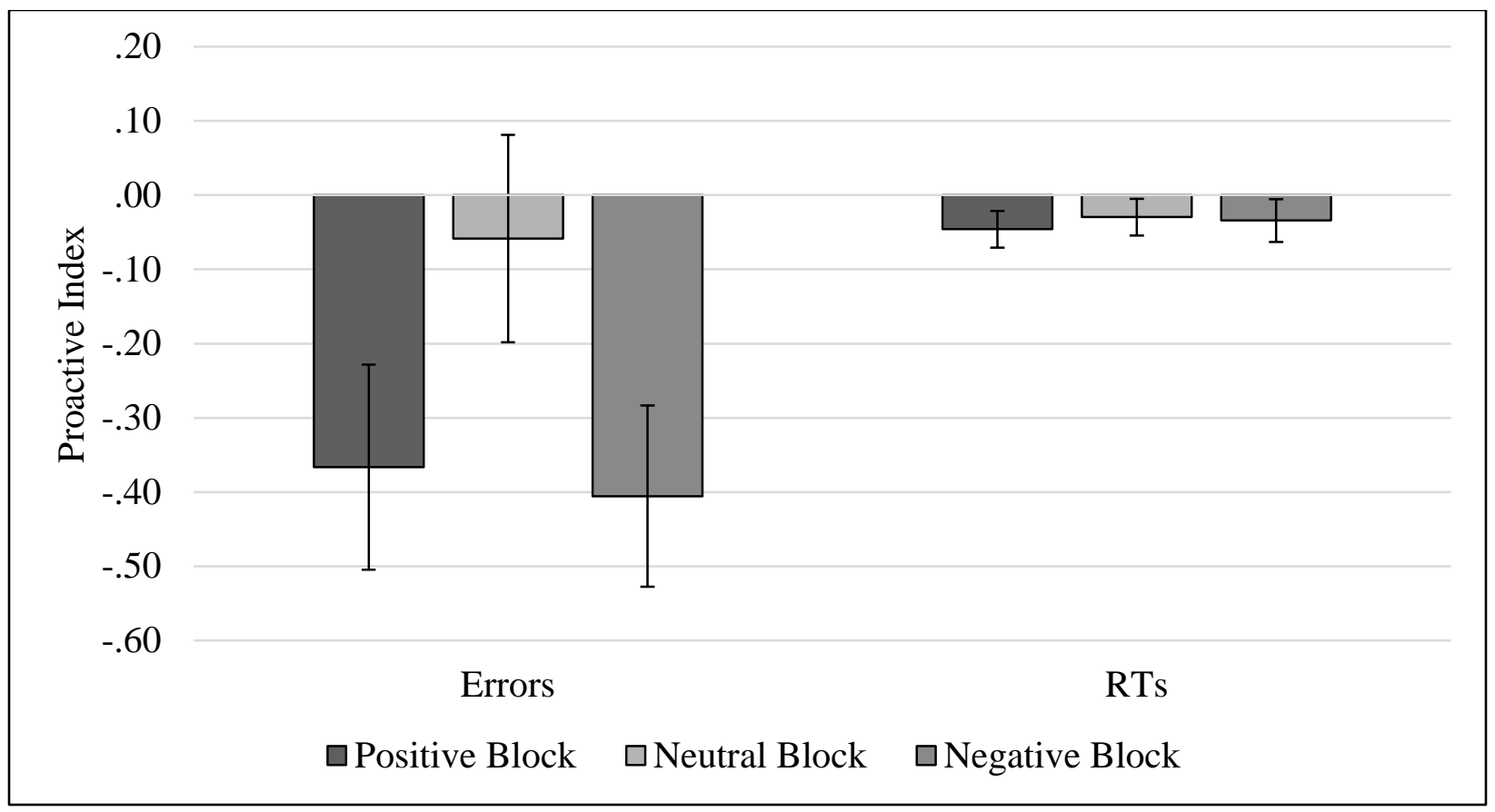

Figure 20. Mean proactive index for errors (left panel) and RTs (right panel) by trial type across the three blocks. Error bars denote standard error.

\section{Individual Differences Measures}

Correlation analyses were conducted to examine the relationship between individual differences (means and standard deviations presented in Table 7) and AX-CPT performance, as indexed by the proactive index for errors and RTs for each block separately. These analyses did not reveal any significant correlations with the proactive index for errors except for a marginally significant positive correlation with Shipley vocabulary scores in the negative block, $r(30)=.36$, $p=.051$. All other correlations were not significant, $p \mathrm{~s}>.082$. Analyses with the proactive index for RTs revealed some significant correlations for the neutral block only: the proactive index positively correlated with digit symbol scores, $r(30)=.42, p=.020$, and with Shipley vocabulary scores, $r(30)=.46, p=.011$. There were no significant correlations in the positive, $p \mathrm{~s}>.168$, or 
negative block, $p s>.066$. Note that all correlations were not corrected for multiple comparisons and should be interpreted with caution.

The Face AX-CPT questionnaire was also coded for common responses (based on $n=25$, because the first five participants did not receive this questionnaire) to gain additional insight into how participants perceived the AX-CPT task. For question 1, 80\% of participants reported that the emotional expression of the faces changed across blocks and 20\% reported that they did not notice any changes. For question 2, 72\% of participants reported that they felt their performance did not change across blocks; $20 \%$ reported a decline in performance due to fatigue; and $8 \%$ felt their performance improved due to practice. For question 3, 68\% reported using a strategy to complete the task (e.g., looking for patterns in the faces presented; however, there were no consistent patterns in the strategies reported) and $8 \%$ reported no strategic approach to the task. Overall, the questionnaire responses suggested that participants noticed that the emotional expressions changed across the blocks but they did not report influences of this manipulation on performance nor did they report consistent use of strategies. 


\section{Discussion}

Experiment 3 examined the effect of emotional face stimuli on older adults' proactive and reactive control. The standard AX-CPT task was modified such that emotional and neutral faces served as cues and neutral faces served as probes. The emotional expression (positive, negative, or neutral) of the valid cue was manipulated across three blocks. Results indicated that older adults used a predominantly reactive strategy to complete the task, as indexed by poorer performance (more errors, increased RTs) on probe-lure than cue-lure trials. This pattern of reactive control in older adults is consistent with results from other studies employing the standard letter AX-CPT task (e.g., Braver et al., 2001, 2005) as well as results from studies using different paradigms (e.g., Stroop and Flanker tasks; Bugg, 2014). Thus, this experiment replicated findings of reactive control in older adults using a paradigm with non-letter stimuli. Inconsistent with hypotheses, performance did not differ across the three emotional and neutral blocks. This suggests that manipulation of the emotional expression of the valid cue face did not affect performance. There was no support for the hypothesis (H 11) that a positive cue face would help older adults better engage in goal maintenance in a proactive manner. There was also no evidence to support the hypothesis that negative cue faces would hinder engagement in proactive control. Rather, there was only a pattern towards reduced reactive control seen in the error data for the neutral block only, as reflected by a numerically larger proactive index value in this block compared to the emotional blocks. However, it is likely that this can be attributed to the sequencing of the blocks. The neutral block was always presented as the second block of trials and had the advantage of practice effects arising from completion of the first block, and is also unlikely to suffer from fatigue effects that are more likely to be present in the third block. Thus, results of this study suggest that older adults' tendency to engage in reactive over proactive 
control is not affected by incorporation of emotional faces or valence manipulation of the cue. This raises the possibility that age-related emotional goals and preferences (e.g., positivity effects) may not function to assist with goal maintenance within a cognitive control task when emotion is not task-relevant. Based on findings that the positivity effect is largest under unconstrained conditions (Reed et al., 2014), it may have been that the AX-CPT task goals did not allow for older adults to naturally engage with the material. In this version, the same face was chosen as the valid cue across all blocks, to be analogous to the valid letter " $\mathrm{X}$ " in the standard letter AX-CPT. Although the expression that was displayed by the valid cue face varied across blocks, it is possible that the repetitiveness may have hindered positivity effects. Future research could try varying the positive stimuli instead.

It is also an open question as to how younger adults would have performed on this task. The literature review demonstrated that younger and older adults sometimes show divergent patterns of emotional stimuli effects. Additionally, many of the studies that have tested the agerelated predictions of the DMC model have used age-group comparisons to support theoretical claims, as opposed to comparing relative differences on probe- and cue-lure trials within an age group (e.g., Braver et al., 2001). Thus, without a younger comparison group, conclusions regarding the effects of emotion (or lack of) on age-related control patterns are limited.

Nonetheless, the results are novel as no other studies have systematically varied emotional stimuli in an AX-CPT paradigm with older adults. Furthermore, the results of Experiment 3 using the face AX-CPT task also contributed insights into the factors underlying enhanced proactive control in the face-letter AX-CPT task. This will be further discussed in the following General Discussion section. 


\section{Chapter 7: General Discussion}

This study aimed to examine the interactions between cognitive control, aging, and emotion. It made novel contributions by elucidating the factors that can modulate age differences in cognitive control. Experiment 1 examined the effect of neutral and negative mood inductions on proactive and reactive control using the standard letter AX-CPT. Typical age-related patterns were found, with younger adults exhibiting proactive control and older adults exhibiting reactive control. Experiment 2 examined the effect of neutral and emotional mood inductions using a modified face-letter AX-CPT that was designed to reduce cognitive demands. Results indicated a strong shift towards proactive and away from reactive control in both age groups, resulting in patterns of age-equivalent proactive control evident in RTs, regardless of induced mood conditions. Finally, Experiment 3 investigated the effect of incorporating emotional and neutral face stimuli in the AX-CPT on older adults' cognitive control. Results revealed typical reactive control patterns that did not vary by emotion. Taken together, the results indicated that cognitive control patterns were most sensitive to the changes of cognitive demands of the task, but showed minimal effects of emotion. Specifically, modifying properties of the AX-CPT task led to enhanced proactive control and a shift away from reactive control. However, the results provided less support of mood or stimulus effects on cognitive control. The following section will highlight the theoretical and empirical implications of the results, in addition to discussing the potential limitations of the study and directions for future investigations.

\section{Age Differences in Cognitive Control}

The DMC framework postulates that there is a shift away from proactive and toward reactive control with advancing age. Results from this study provide further evidence to support this proposition. There was converging evidence of proactive control in younger adults and 
reactive control in older adults, replicated across five separate groups of participants (two younger and three older adult groups in Experiments 1 and 3). This strongly supports the predictions of age-related control patterns outlined in the DMC model and demonstrates the ability of this theory to specify the particular cognitive control deficit (i.e., proactive control) that is apparent in older age ${ }^{12}$.

It was also evident in this study that age-related patterns of control are flexible and amenable to change, as has been previously demonstrated through methods such as strategy training (Braver et al., 2009; Paxton et al., 2006). Experiment 2 contributed novel and clear evidence that changing certain task parameters of the AX-CPT can also modulate age-related patterns. Specifically, the face-letter AX-CPT caused a clear shift away from reactive and toward proactive control in the RT data, particularly in older adults. Although the exact modifications and mechanisms underlying this shift are not clear, results from the face AX-CPT used in Experiment 3 provide some insights. This version of the AX-CPT was designed with the hypothesis that face cues, which are more salient and meaningful than letters, would help facilitate context representation and maintenance in older adults (through enhanced cue processing), which would lead to enhanced proactive control. However, despite using only face stimuli in the face AX-CPT, older adults only exhibited reactive control, with no evidence of proactive control. This suggests that the inclusion of faces per se was likely not the critical factor

\footnotetext{
${ }^{12}$ Although it is outside the scope of this dissertation to compare the DMC to other theories of cognitive control and accounts of age-related declines (see Braver et al., 2001, 2007, for such a comparison), it is noteworthy to mention that the specific patterns observed in this study cannot readily be explained or fully accounted for by some prominent theories of cognitive aging. For example, inhibitory deficit theory (Hasher \& Zacks, 1988) posits that aging results in a decline of the ability to inhibit irrelevant distractor information. Thus, this theory would predict that older adults would show poor inhibition on probe-lure trials but cannot readily explain why they perform better than younger adults on cue-lure trials. The DMC model can account for these patterns by specifying that the latter difference arises from a specific impairment to context processing in older adults. This better accounts for the age differences on both probe- and cue-lure trials.
} 
underlying enhanced proactive control in the face-letter AX-CPT in Experiment 2. Instead, more plausible explanations of the shift toward proactive control include: (a) the marked distinction between salient and complex face cues versus simple letter probes is necessary, as this is likely to direct processing towards the cue and away from the probe; or (b) the need to make twochoice semantic judgements (regarding gender) about the face cue facilitated deeper processing of the cue/contextual information, thus strengthening context representations. Future research can modify the two novel variants of the AX-CPT tested in this study (e.g., incorporating nonface stimuli, manipulating different levels of processing) to further pinpoint the critical mechanisms underlying the shift toward proactive control.

\section{Emotion and Cognitive Control}

Research has found that cognitive control in younger adults can be affected by emotion. Specifically, studies have found evidence of reduced proactive control under positive mood inductions (Fröber \& Dreisbach, 2012) and when the task contains positive stimuli (Dreisbach, 2006). However, Experiment 2B did not find evidence of changes to proactive control in younger adults induced into positive moods. Some speculative explanations may be that the positive induction was too mild and not effective enough to elicit changes to proactive control. The limitations of mood inductions will be discussed below. Additionally, as previous studies have presented positive stimuli on a trial-by-trial basis and found reduced proactive control, it may be the case that transient positive affect needs to be maintained with continuously presented positive items in order for effects to emerge. There were also no effects of negative mood on cognitive control in younger adults. This is more consistent with results from other studies that also did not find any effects of negative stimuli (Fröber \& Dreisbach, 2012). As discussed earlier, the lack of effects on cognitive control from negative emotion appears to be consistent in the literature and it 
has been suggested that only positive emotions will have substantial effects on proactive control due to the relationship between positive emotion and dopamine (Mitchell \& Phillips, 2007).

\section{Aging, Emotion, and Cognitive Control}

The SST argues that advanced age is associated with a limited perspective, resulting in motivations to prioritize positive wellbeing. This has been evidenced through studies demonstrating positivity effects (Reed et al., 2014). It was hypothesized that these motivational shifts would cause different patterns of emotional effects on cognitive control in younger and older adults. Contrary to hypotheses, there was insufficient evidence to suggest that mood or emotional stimuli have significant effects on age-related patterns of proactive and reactive control. Most surprisingly, there was no evidence of a positivity effect on cognitive control as hypothesized - older adults did not differ from younger adults on effects of positive mood induction nor did they show evidence of greater processing toward positive face cues. Although this study made novel contributions and added to the very limited literature investigating mood and emotional stimuli effects on cognitive control in older populations, it did not reveal significant effects of emotion. This conflicts with other studies that have found emotion effects in older adults on processes that are presumably related to cognitive control (e.g., Biss et al., 2012; Carpenter et al., 2013; Chu, 2014). However, there was some limited evidence of a mood by age interaction. Most noteworthy, there was some evidence of enhanced proactive control (indexed by longer RTs for cue-lure trials) in older adults induced into negative relative to positive and neutral moods. This result was not due to generally slower responses in the negative induction group specifically (i.e., RTs for the other trials were similar across mood groups) or a speed accuracy trade-off. The results of this study and the lack of studies examining the effect of negative mood on older adults' cognitive control can only provide speculative explanations for 
this result. One explanation (detailed in Experiment 2B, Discussion) that potentially warrants follow-up research is the idea that older adults in negative moods are motivated to repair moods and may seek out information that could help with this, such as enhanced attention toward socially meaningful faces. This enhanced and controlled attention toward the cue faces may have helped promote context processing, leading older adults to be slower on cue-lure trials. It should be highlighted that in the face-letter AX-CPT, faces were all neutral. A potential follow-up investigation would be to use positive faces in combination with negative inductions, similar to studies by Isaacowitz and colleagues (e.g., Isaacowitz, Toner, Goren, \& Wilson, 2008; Isaacowitz et al., 2009) to test this hypothesis empirically.

\section{Limitations}

There are several potential limitations that should be noted. First, this study used only the AX-CPT to assess proactive and reactive control. The AX-CPT was selected as it is the standard task associated with the DMC framework and has established construct validity (Braver et al., 2005). Although multiple variants of this task were used across the various experiments, this study would have benefited from the inclusion of other tasks that may also distinguish between the two control modes. Some potential tasks that have been used in other studies include the Stroop task (e.g., Bugg, 2014) and task-switching paradigms (e.g., Czernochowski, Nessler, \& Friedman, 2010). Similar results across multiple measures would have provided converging evidence and thus allow for stronger conclusions. Indeed, a latent variable approach has been advocated to further examine behavioural markers of proactive and reactive control (Braver, 2012). However, the primary focus of this study was to investigate the interactions between cognitive control, aging and emotion; thus, it did not attempt to venture beyond this already established measure. 
Other potential limitations of this study concern the choice of mood induction procedure and mood measures. Although the intent of the induction slide show was to induce low-arousal moods, it is possible that the induced moods were too mild. This may have limited the ability to observe influences of mood on cognitive control. It is possible that the induction of higher arousal states would have produced different results. Additionally, self-reports of mood were collected at multiple time points but not while participants were completing the AX-CPT. Thus, it is not known what moods participants were experiencing; we can only assume that participants completed the block under the mood that they reported just prior to beginning a block. It is also not clear how long the moods lasted. Across experiments and across induction groups, it was observed that younger adults had a general tendency to report lower moods across time. Older adults also had a tendency to return to baseline levels faster. Thus, it is possible that the effects of the induction may have been relatively short-lived, particularly for older adults, which hinders conclusions regarding the effect of mood on cognitive control ${ }^{13}$. Psychophysiological measures of mood (e.g., heart rate or electromyography) would have been advantageous to help address these issues. Although these measures are generally able to distinguish between high and low arousal states, these measures are not yet able to reliably distinguish between positive and negative moods.

There are other conceptual and practical issues to consider when examining mood through inductions. For example, there is a trade-off between experimental control and external validity. Attempts to control for potential confounding variables present in the induction procedures (e.g., time, type of content used) likely reduce external validity. Thus, despite reports

\footnotetext{
${ }^{13}$ Separate analyses on the first and second AX-CPT blocks revealed the same patterns as the reported analyses on both blocks.
} 
of mood states changing due to the inductions, it is unclear how accurately these reported states in the laboratory reflect moods that occur in more complex real-life situations. Additionally, due to ethical concerns, mood inductions are also likely to induce relatively mild and transient mood states. These issues limit the generalizability of any mood effects found using these experimental inductions. There is also the practical issue of participant expectancy effects if participants are aware that the inductions are meant to induce a certain mood state and their mood ratings may reflect these expectancies, rather than reporting their actual moods. Despite these issues, mood inductions are currently the standard for effective experimental manipulations of mood in order to examine the effects on cognitive abilities. They appear to be effective at demonstrating differential influences of emotional versus neutral mood inductions on cognition and thus provide value for informing mood effects.

Finally, it should also be noted that this study examined the effect of mood using a between-subjects design. Although participants were randomly assigned to induction conditions, it is possible that pre-existing group differences in cognitive control (e.g., those not captured by the measures of individual differences administered in this study) may have contributed to performance differences. Ideally, future research should examine performance changes by employing a pre- versus post-induction design. This design was not used in the current study to minimize concerns regarding fatigue (e.g., Chiew \& Braver, 2014) and confounding practice effects (Paxton et al., 2006).

\section{Future Directions}

In addition to the potential follow-up studies described earlier, there are many avenues for future research examining the interactions between aging, "cold” cognitive control, and "hot” emotional functioning. Most notably, results from this study contributed evidence of the factors 
that can be modified to facilitate proactive control in older adults. Future work in this area may help identify compensatory mechanisms or environmental modifications that could be applied to everyday functioning. Elucidating factors that can attenuate age-related declines has valuable implications for interventions aimed at improving cognitive functioning in older adults. A promising method is to use neuroimaging techniques, such as functional magnetic resonance imaging (fMRI) or electroencephalography (EEG), which have shown clear and interesting patterns of cognitive control and emotion in the brain (e.g., Braver et al., 2009; Schmitt et al., 2015). The current study only used behavioural measures and it may have been possible that these measures were not sensitive enough to detect any emotion effects, especially subtle ones that may have been present. Researchers studying how cognitive control is affected by emotion in younger adults (e.g., Dreisbach, 2006) and motivation in older adults (e.g., Schmitt et al., 2015) using neuroimaging have found that these techniques provide valuable contributions, above and beyond that of behavioural measures.

Undoubtedly, it is apparent that investigations of the interactions between cognitive control, aging, and emotion can provide promising contributions to each individual research domain (Braver et al., 2014). Together, work that combines these areas produces a powerful and richer account of "cold" cognitive control that encompasses the unique qualities and changes that accompany the "hot" emotional profile of aging. 
Appendix I: Ryerson REB Approval Letter (REB 2013-286)

\section{RYERSONUNIVERSITY}

\section{RESEARCH ETHICS BOARD}

To: Linda Truong

Psychology

Re: REB 2013-286: Age, Cognitive Control, and Emotion

Date: November 4, 2013

\section{Dear Linda Truong,}

The review of your protocol REB File REB 2013-286 is now complete. The project has been approved for a one year period. Please note that before proceeding with your project, compliance with other required University approvals/certifications, institutional requirements, or governmental authorizations may be required.

This approval may be extended after one year upon request. Please be advised that if the project is not renewed, approval will expire and no more research involving humans may take place. If this is a funded project, access to research funds may also be affected.

Please note that REB approval policies require that you adhere strictly to the protocol as last reviewed by the REB and that any modifications must be approved by the Board before they can be implemented. Adverse or unexpected events must be reported to the REB as soon as possible with an indication from the Principal Investigator as to how, in the view of the Principal Investigator, these events affect the continuation of the protocol.

Finally, if research subjects are in the care of a health facility, at a school, or other institution or community organization, it is the responsibility of the Principal Investigator to ensure that the ethical guidelines and approvals of those facilities or institutions are obtained and filed with the REB prior to the initiation of any research.

Please quote your REB file number (REB 2013-286) on future correspondence.

Congratulations and best of luck in conducting your research.

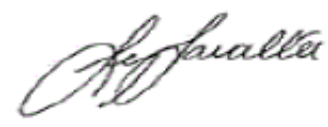

Lynn Lavallée, Ph.D.

Chair, Research Ethics Board 
Appendix II: Consent Form-Younger Adults (Experiment 1)

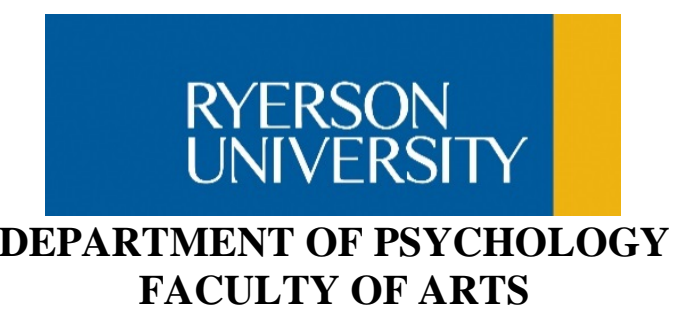

\section{CONSENT TO PARTICIPATE IN RESEARCH} Aging, Cognitive Control, and Emotion-I

Principal Investigator: Lixia Yang, PhD; Email: lixiay@psych.ryerson.ca

Student Researcher: Linda Truong, MA; Email: ltruong@psych.ryerson.ca

Lab Phone: (416) 979-5000 ext. 4987

You are being invited to participate in a research study. Please read this Consent Form so that you understand what your participation will involve. Before you consent to participate, please ask any questions necessary to be sure you understand what your participation will involve.

\section{Investigators}

This research study is being conducted by Linda Truong and Dr. Lixia Yang, from the Department of Psychology at Ryerson University. The results of this study will contribute to Linda Truong's doctoral dissertation. This study is sponsored by a Discovery Grant from the Natural Sciences and Engineering Research Council of Canada (NSERC). If you have any questions or concerns about the research, please feel free to contact: Dr. Lixia Yang at 416-9795000 ext. 6522 or lixiay@psych.ryerson.ca or Linda Truong at 416-979-5000 ext. 4987 or ltruong@psych.ryerson.ca.

\section{Purpose of the Study}

This experiment examines how aging and emotion influences cognitive control. Cognitive control is the ability to keep in mind the goals of a task. The performance of younger and older adults on cognitive control tasks will be compared to examine how this ability changes with age and how it is influenced by emotional mood and/or emotional items. Younger adult participants will be students who are enrolled in the Introduction to Psychology (PSY102/202) courses. A total of 72 younger adults will be recruited for this study.

\section{Description of the Study and Your Participation}

If you volunteer to participate in this study, you will be asked to do the following things: complete self-report questionnaires on mood, listen to music/noise, view emotional images, complete computerized and paper-and-pencil tasks assessing thinking abilities, and complete demographic and health questionnaires that ask for information such as age, gender, and past and current health status. We request this information in order to assess whether these factors may have effects on the tasks we ask you to perform. In accordance with the Personal Health Information Protection Act, any health information will be collected, used, stored and shared in a manner that protects the confidentiality and privacy of individuals. You also have the right to 
withhold this information, and refuse to answer any questions or tasks, without consequence and you will still receive your allotted course credit. Prior to all tasks, the procedure will be explained and you will have an opportunity to ask questions. Participation in the entire study will take approximately 1.5 hours to complete. It will take place at the Psychology Research and Training Centre of Ryerson University (105 Bond St.), with experimenters from the Cognitive Aging Laboratory. Individual research findings will not be available for distribution; however, group results can be requested by participants and will be provided by mail or email.

\section{Potential Risks and Discomforts}

There is minimal risk anticipated in this study. However, completing tasks that assess cognitive and emotional functioning can be a source of discomfort for some participants. If you experience discomfort or distress, you have the right to refrain from answering a question or completing a task. You can also discontinue your participation at any point during the experiment. You will still receive your allotted course credit.

\section{Potential Benefits to Participants and/or to Society}

Through your participation, you will have the benefit of contributing to and learning about psychological research. Additionally, this study has the potential to contribute to scientific research and knowledge on how aging influences cognitive and emotional processing. I cannot guarantee, however, that you will receive any benefits from participating in this study.

\section{Compensation for Participation}

You will be compensated 1.5 course credits for participation in this study. You will also have an option for a "walk through" session by completing some or all of the tasks but with the choice of withdrawing your data from the final data set. Compensation for your time will be provided even if you do not complete the study or choose to "walk through" the session.

\section{Confidentiality}

Maintaining confidentiality of our participants is important to us. For this reason, a participant identification code will be assigned to your data. Only qualified personnel, including investigators and research assistants, will have access to this data. All files will be stored and locked in a secure place in the Cognitive Aging Laboratory at Ryerson University, and will be kept for up to ten years after the publication of the data.

\section{Voluntary Participation and Withdrawal}

Participation in this study is voluntary. You can choose whether to be in this study or not. If you volunteer to be in this study, you may withdraw at any time without consequences of any kind. If you choose to withdraw from this study you may also choose to withdraw your data from the study. You may also choose not to answer any question(s) and still remain in the study. Your choice of whether or not to participate will not influence your future relations with Ryerson University.

\section{Questions about the Study}

If you have any questions about the research now, please ask. If you have any questions about the research, you may contact Linda Truong at 416-979-5000, ext. 4987 or

ltruong@psych.ryerson.ca. 
This study has been reviewed by the Ryerson University Research Ethics Board. If you have questions regarding your rights as a research participant in this study, please contact:

\author{
Lynn Lavallée, Ph.D. \\ Associate Professor \\ Chair, Research Ethics Board \\ Office of the Vice President, Research and Innovation \\ Ryerson University \\ 350 Victoria Street \\ Toronto, ON, M5B 2K3 \\ Telephone: (416) 9795000 x 4791 \\ Email: lavallee@ryerson.ca
}

\title{
Signature of Research Participant/Legal Representative
}

Your signature below indicates that you have read the information in this agreement and have had a chance to ask any questions you have about the study Aging, Cognitive Control, and Emotion-I as described herein. Your questions have been answered to your satisfaction. Your signature also indicates that you agree to participate in this study and have been told that you can change your mind and withdraw your consent to participate at any time. You have been given a copy of this agreement. You have been told that by signing this consent agreement you are not giving up any of your legal rights.

Name of the Participant (please print)

Signature of the Participant

Date

Signature of Investigator

Date 
Appendix III: Consent Form-Older Adults (Experiments 1 \& 2)

RYERSON

UNIVERSITY

\section{DEPARTMENT OF PSYCHOLOGY \\ FACULTY OF ARTS}

\section{CONSENT TO PARTICIPATE IN RESEARCH}

Aging, Cognitive Control, and Emotion-I

Principal Investigator: Lixia Yang, PhD; Email: lixiay@psych.ryerson.ca

Student Researcher: Linda Truong, MA; Email: ltruong@psych.ryerson.ca

Lab Phone: (416) 979-5000 ext. 4987

You are being invited to participate in a research study. Please read this Consent Form so that you understand what your participation will involve. Before you consent to participate, please ask any questions necessary to be sure you understand what your participation will involve.

\section{Investigators}

This research study is being conducted by Linda Truong and Dr. Lixia Yang, from the Department of Psychology at Ryerson University. The results of this study will contribute to Linda Truong's doctoral dissertation. This study is sponsored by a Discovery Grant from the Natural Sciences and Engineering Research Council of Canada (NSERC). If you have any questions or concerns about the research, please feel free to contact: Dr. Lixia Yang at 416-9795000 ext. 6522 or lixiay@psych.ryerson.ca or Linda Truong at 416-979-5000 ext. 4987 or ltruong@psych.ryerson.ca.

\section{Purpose of the Study}

This experiment examines how aging and emotion influences cognitive control. Cognitive control is the ability to keep in mind the goals of a task. The performance of younger and older adults on cognitive control tasks will be compared to examine how this ability changes with age and how it is influenced by emotional mood and/or emotional items.

\section{Description of the Study and Your Participation}

If you volunteer to participate in this study, you will be asked to do the following things: complete self-report questionnaires on mood, listen to music/noise, view emotional images, complete computerized and paper-and-pencil tasks assessing thinking abilities, and complete demographic and health questionnaires that ask for information such as age, gender, and past and current health status. We request this information in order to assess whether these factors may have effects on the tasks we ask you to perform. In accordance with the Personal Health Information Protection Act, any health information will be collected, used, stored and shared in a manner that protects the confidentiality and privacy of individuals. You also have the right to withhold this information, and refuse to answer any questions or tasks, without consequence. Prior to all tasks, the procedure will be explained and you will have an opportunity to ask questions. Participation in the entire study will take up to two hours to complete. It will take 
place at the Psychology Research and Training Centre of Ryerson University (105 Bond St.), with experimenters from the Cognitive Aging Laboratory. Individual research findings will not be available for distribution; however, group results can be requested by participants and will be provided by mail or email.

\section{Potential Risks and Discomforts}

There is minimal risk anticipated in this study. However, completing tasks that assess cognitive and emotional functioning can be a source of discomfort for some participants. If you experience discomfort or distress, you have the right to refrain from answering a question or completing a task. You can also discontinue your participation at any point during the experiment.

\section{Potential Benefits to Participants and/or to Society}

Through your participation, you will have the benefit of contributing to and learning about psychological research. Additionally, this study has the potential to contribute to scientific research and knowledge on how aging influences cognitive and emotional processing. I cannot guarantee, however, that you will receive any benefits from participating in this study.

\section{Payment for Participation}

You will be compensated $\$ 20$ in cash for participation in this study. You will also have an option for a "walk through" session by completing some or all of the tasks but with the choice of withdrawing your data from the final data set. Compensation for your time will be provided even if you do not complete the study or choose to "walk through" the session.

\section{Confidentiality}

Maintaining confidentiality of our participants is important to us. For this reason, a participant identification code will be assigned to your data. Only qualified personnel, including investigators and research assistants, will have access to this data. All files will be stored and locked in a secure place in the Cognitive Aging Laboratory at Ryerson University, and will be kept for up to ten years after the publication of the data.

\section{Voluntary Participation and Withdrawal}

Participation in this study is voluntary. You can choose whether to be in this study or not. If you volunteer to be in this study, you may withdraw at any time without consequences of any kind. If you choose to withdraw from this study you may also choose to withdraw your data from the study. You may also choose not to answer any question(s) and still remain in the study. Your choice of whether or not to participate will not influence your future relations with Ryerson University.

\section{Questions about the Study}

If you have any questions about the research now, please ask. If you have any questions about the research, you may contact Linda Truong at 416-979-5000, ext. 4987 or ltruong@psych.ryerson.ca. 
This study has been reviewed by the Ryerson University Research Ethics Board. If you have questions regarding your rights as a research participant in this study, please contact:

\author{
Lynn Lavallée, Ph.D. \\ Associate Professor \\ Chair, Research Ethics Board \\ Office of the Vice President, Research and Innovation \\ Ryerson University \\ 350 Victoria Street \\ Toronto, ON, M5B 2K3 \\ Telephone: (416) 9795000 x 4791 \\ Email: lavallee@ryerson.ca
}

\title{
Signature of Research Participant/Legal Representative
}

Your signature below indicates that you have read the information in this agreement and have had a chance to ask any questions you have about the study Aging, Cognitive Control, and Emotion-I as described herein. Your questions have been answered to your satisfaction. Your signature also indicates that you agree to participate in this study and have been told that you can change your mind and withdraw your consent to participate at any time. You have been given a copy of this agreement. You have been told that by signing this consent agreement you are not giving up any of your legal rights.

Name of the Participant (please print)

Signature of the Participant

Date

Signature of Investigator

Date 
Appendix IV: Debriefing Form (Experiment 1)

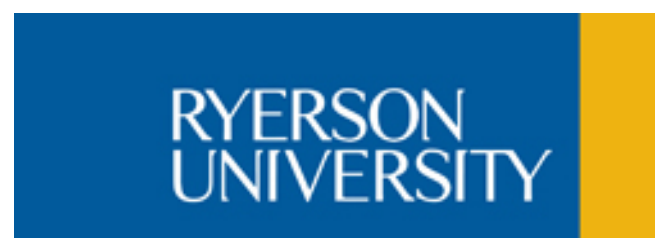

\title{
DEPARTMENT OF PSYCHOLOGY FACULTY OF ARTS
}

\author{
Debriefing \\ Aging, Cognitive Control, and Emotion-I
}

Thank you for your participation!

In this study, we examined how aging and emotion affect cognitive control. Cognitive control is your ability to keep in mind the goals of a task and to adjust behaviour to successfully accomplish these goals. Prior research has suggested that this ability declines as you age. However, your ability to regulate and process emotion or emotional information remains stable, or even improves, with age. This study aimed to examine how emotional functioning influences cognitive control in younger versus older adults. Participants were induced into certain moods (e.g., happy, sad, or neutral) prior to completing a cognitive control task. It is hypothesized that younger and older adults will perform differently, depending on what mood they experienced during the task. Younger adults are expected to perform best when induced into negative moods. In contrast, the performance of older adults is expected to benefit from positive moods. Such results would be consistent with previous research on the topic.

Your participation in this study contributes to the growing research on interactions between aging, cognitive control, and emotion. It will help us better understand the factors that can help support cognitive control in healthy aging adults.

Please do not hesitate to ask the experimenter questions or to raise any concerns you have. Feel free to contact the principal investigators, Linda Truong at 416-979-5000 ext. 4987 and Dr. Lixia Yang at 416-979-5000 ext. 6522, if you have any questions regarding this project. You can also contact the Chair of the Ryerson Research Ethics Board, Lynn Lavallée, Ph.D., at 416-979-5000 ext. 4791. We greatly appreciate your time and participation! 
Appendix V: Consent Form-Younger Adults (Experiment 2)

RYERSON

UNIVERSITY

\section{DEPARTMENT OF PSYCHOLOGY}

FACULTY OF ARTS

\section{CONSENT TO PARTICIPATE IN RESEARCH}

Aging, Cognitive Control, and Emotion-I

Principal Investigator: Lixia Yang, PhD; Email: lixiay@psych.ryerson.ca

Student Researcher: Linda Truong, MA; Email: ltruong@psych.ryerson.ca

Lab Phone: (416) 979-5000 ext. 4987

You are being invited to participate in a research study. Please read this Consent Form so that you understand what your participation will involve. Before you consent to participate, please ask any questions necessary to be sure you understand what your participation will involve.

\section{Investigators}

This research study is being conducted by Linda Truong and Dr. Lixia Yang, from the Department of Psychology at Ryerson University. The results of this study will contribute to Linda Truong's doctoral dissertation. This study is sponsored by a Discovery Grant from the Natural Sciences and Engineering Research Council of Canada (NSERC). If you have any questions or concerns about the research, please feel free to contact: Dr. Lixia Yang at 416-9795000 ext. 6522 or lixiay@psych.ryerson.ca or Linda Truong at 416-979-5000 ext. 4987 or ltruong@psych.ryerson.ca.

\section{Purpose of the Study}

This experiment examines how aging and emotion influences cognitive control. Cognitive control is the ability to keep in mind the goals of a task. The performance of younger and older adults on cognitive control tasks will be compared to examine how this ability changes with age and how it is influenced by emotional mood and/or emotional items. Younger adult participants will be students who are enrolled in the Introduction to Psychology (PSY102/202) courses. A total of 72 younger adults will be recruited for this study.

\section{Description of the Study and Your Participation}

If you volunteer to participate in this study, you will be asked to do the following things: complete self-report questionnaires on mood, listen to music/noise, view emotional images, complete computerized and paper-and-pencil tasks assessing thinking abilities, and complete demographic and health questionnaires that ask for information such as age, gender, and past and current health status. We request this information in order to assess whether these factors may have effects on the tasks we ask you to perform. In accordance with the Personal Health Information Protection Act, any health information will be collected, used, stored and shared in a manner that protects the confidentiality and privacy of individuals. You also have the right to withhold this information, and refuse to answer any questions or tasks, without consequence and 
you will still receive your allotted course credit. Prior to all tasks, the procedure will be explained and you will have an opportunity to ask questions. Participation in the entire study will take approximately 1.5 hours to complete. It will take place at the Psychology Research and Training Centre of Ryerson University (105 Bond St.), with experimenters from the Cognitive Aging Laboratory. Individual research findings will not be available for distribution; however, group results can be requested by participants and will be provided by mail or email.

\section{Potential Risks and Discomforts}

There is minimal risk anticipated in this study. However, completing tasks that assess cognitive and emotional functioning can be a source of discomfort for some participants. If you experience discomfort or distress, you have the right to refrain from answering a question or completing a task. You can also discontinue your participation at any point during the experiment. You will still receive your allotted course credit.

\section{Potential Benefits to Participants and/or to Society}

Through your participation, you will have the benefit of contributing to and learning about psychological research. Additionally, this study has the potential to contribute to scientific research and knowledge on how aging influences cognitive and emotional processing. I cannot guarantee, however, that you will receive any benefits from participating in this study.

\section{Compensation for Participation}

You will be compensated 1.5 course credits for participation in this study. You will also have an option for a "walk through" session by completing some or all of the tasks but with the choice of withdrawing your data from the final data set. Compensation for your time will be provided even if you do not complete the study or choose to "walk through" the session.

\section{Confidentiality}

Maintaining confidentiality of our participants is important to us. For this reason, a participant identification code will be assigned to your data. Only qualified personnel, including investigators and research assistants, will have access to this data. All files will be stored and locked in a secure place in the Cognitive Aging Laboratory at Ryerson University, and will be kept for up to ten years after the publication of the data.

\section{Voluntary Participation and Withdrawal}

Participation in this study is voluntary. You can choose whether to be in this study or not. If you volunteer to be in this study, you may withdraw at any time without consequences of any kind. If you choose to withdraw from this study you may also choose to withdraw your data from the study. You may also choose not to answer any question(s) and still remain in the study. Your choice of whether or not to participate will not influence your future relations with Ryerson University.

\section{Questions about the Study}

If you have any questions about the research now, please ask. If you have any questions about the research, you may contact Linda Truong at 416-979-5000, ext. 4987 or ltruong@psych.ryerson.ca. 
This study has been reviewed by the Ryerson University Research Ethics Board. If you have questions regarding your rights as a research participant in this study, please contact:

\author{
Lynn Lavallée, Ph.D. \\ Associate Professor \\ Chair, Research Ethics Board \\ Office of the Vice President, Research and Innovation \\ Ryerson University \\ 350 Victoria Street \\ Toronto, ON, M5B 2K3 \\ Telephone: (416) 9795000 x 4791 \\ Email: lavallee@ryerson.ca
}

\title{
Signature of Research Participant/Legal Representative
}

Your signature below indicates that you have read the information in this agreement and have had a chance to ask any questions you have about the study Aging, Cognitive Control, and Emotion-I as described herein. Your questions have been answered to your satisfaction. Your signature also indicates that you agree to participate in this study and have been told that you can change your mind and withdraw your consent to participate at any time. You have been given a copy of this agreement. You have been told that by signing this consent agreement you are not giving up any of your legal rights.

Name of the Participant (please print)

Signature of the Participant

Date

Signature of Investigator

Date 
Appendix VI: Debriefing Form (Experiment 2)

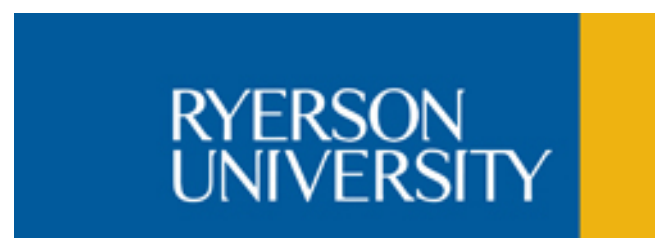

\title{
DEPARTMENT OF PSYCHOLOGY FACULTY OF ARTS
}

\author{
Debriefing \\ Aging, Cognitive Control, and Emotion-I
}

Thank you for your participation!

In this study, we examined how aging and emotion affect cognitive control. Cognitive control is your ability to keep in mind the goals of a task and to adjust behaviour to successfully accomplish these goals. Prior research has suggested that this ability declines as you age. However, your ability to regulate and process emotion or emotional information remains stable, or even improves, with age. This study aimed to examine how emotional functioning influences cognitive control in younger versus older adults. Participants were induced into certain moods (e.g., happy, sad, or neutral) prior to completing a cognitive control task. It is hypothesized that younger and older adults will perform differently, depending on what mood they experienced during the task. Younger adults are expected to perform best when induced into negative moods. In contrast, the performance of older adults is expected to benefit from positive moods. Such results would be consistent with previous research on the topic.

Your participation in this study contributes to the growing research on interactions between aging, cognitive control, and emotion. It will help us better understand the factors that can help support cognitive control in healthy aging adults.

Please do not hesitate to ask the experimenter questions or to raise any concerns you have. Feel free to contact the principal investigators, Linda Truong at 416-979-5000 ext. 4987 and Dr. Lixia Yang at 416-979-5000 ext. 6522, if you have any questions regarding this project. You can also contact the Chair of the Ryerson Research Ethics Board, Lynn Lavallée, Ph.D., at 416-979-5000 ext. 4791. We greatly appreciate your time and participation! 
Appendix VII: Consent Form (Experiment 3)

RYERSON

UNIVERSITY

\section{DEPARTMENT OF PSYCHOLOGY \\ FACULTY OF ARTS}

\section{CONSENT TO PARTICIPATE IN RESEARCH}

Aging, Cognitive Control, and Emotion-I

Principal Investigator: Lixia Yang, PhD; Email: lixiay@psych.ryerson.ca

Student Researcher: Linda Truong, MA; Email: ltruong@psych.ryerson.ca

Lab Phone: (416) 979-5000 ext. 4987

You are being invited to participate in a research study. Please read this Consent Form so that you understand what your participation will involve. Before you consent to participate, please ask any questions necessary to be sure you understand what your participation will involve.

\section{Investigators}

This research study is being conducted by Linda Truong and Dr. Lixia Yang, from the Department of Psychology at Ryerson University. The results of this study will contribute to Linda Truong's doctoral dissertation. This study is sponsored by a Discovery Grant from the Natural Sciences and Engineering Research Council of Canada (NSERC). If you have any questions or concerns about the research, please feel free to contact: Dr. Lixia Yang at 416-9795000 ext. 6522 or lixiay@psych.ryerson.ca or Linda Truong at 416-979-5000 ext. 4987 or ltruong@psych.ryerson.ca.

\section{Purpose of the Study}

This experiment examines how aging and emotion influences cognitive control. Cognitive control is the ability to keep in mind the goals of a task. The performance of younger and older adults on cognitive control tasks will be compared to examine how this ability changes with age and how it is influenced by emotional items.

\section{Description of the Study and Your Participation}

If you volunteer to participate in this study, you will be asked to do the following things: complete self-report questionnaires on mood, view emotional images, complete computerized and paper-and-pencil tasks assessing thinking abilities, and complete demographic and health questionnaires that ask for information such as age, gender, and past and current health status. We request this information in order to assess whether these factors may have effects on the tasks we ask you to perform. In accordance with the Personal Health Information Protection Act, any health information will be collected, used, stored and shared in a manner that protects the confidentiality and privacy of individuals. You also have the right to withhold this information, and refuse to answer any questions or tasks, without consequence. Prior to all tasks, the procedure will be explained and you will have an opportunity to ask questions. Participation in the entire study will take approximately one hour to complete. It will take place at the 
Psychology Research and Training Centre of Ryerson University (105 Bond St.), with experimenters from the Cognitive Aging Laboratory. Individual research findings will not be available for distribution; however, group results can be requested by participants and will be provided by mail or email.

\section{Potential Risks and Discomforts}

There is minimal risk anticipated in this study. However, completing tasks that assess cognitive and emotional functioning can be a source of discomfort for some participants. If you experience discomfort or distress, you have the right to refrain from answering a question or completing a task. You can also discontinue your participation at any point during the experiment.

\section{Potential Benefits to Participants and/or to Society}

Through your participation, you will have the benefit of contributing to and learning about psychological research. Additionally, this study has the potential to contribute to scientific research and knowledge on how aging influences cognitive and emotional processing. I cannot guarantee, however, that you will receive any benefits from participating in this study.

\section{Payment for Participation}

You will be compensated \$12/hour in cash for participation in this study. You will also have an option for a "walk through" session by completing some or all of the tasks but with the choice of withdrawing your data from the final data set. Compensation for your time will be provided even if you do not complete the study or choose to "walk through" the session.

\section{Confidentiality}

Maintaining confidentiality of our participants is important to us. For this reason, a participant identification code will be assigned to your data. Only qualified personnel, including investigators and research assistants, will have access to this data. All files will be stored and locked in a secure place in the Cognitive Aging Laboratory at Ryerson University, and will be kept for up to ten years after the publication of the data.

\section{Voluntary Participation and Withdrawal}

Participation in this study is voluntary. You can choose whether to be in this study or not. If you volunteer to be in this study, you may withdraw at any time without consequences of any kind. If you choose to withdraw from this study you may also choose to withdraw your data from the study. You may also choose not to answer any question(s) and still remain in the study. Your choice of whether or not to participate will not influence your future relations with Ryerson University.

\section{Questions about the Study}

If you have any questions about the research now, please ask. If you have any questions about the research, you may contact Linda Truong at 416-979-5000, ext. 4987 or ltruong@psych.ryerson.ca. 
This study has been reviewed by the Ryerson University Research Ethics Board. If you have questions regarding your rights as a research participant in this study, please contact:

\author{
Lynn Lavallée, Ph.D. \\ Associate Professor \\ Chair, Research Ethics Board \\ Office of the Vice President, Research and Innovation \\ Ryerson University \\ 350 Victoria Street \\ Toronto, ON, M5B 2K3 \\ Telephone: (416) 9795000 x 4791 \\ Email: lavallee@ryerson.ca
}

\title{
Signature of Research Participant/Legal Representative
}

Your signature below indicates that you have read the information in this agreement and have had a chance to ask any questions you have about the study Aging, Cognitive Control, and Emotion-II as described herein. Your questions have been answered to your satisfaction. Your signature also indicates that you agree to participate in this study and have been told that you can change your mind and withdraw your consent to participate at any time. You have been given a copy of this agreement. You have been told that by signing this consent agreement you are not giving up any of your legal rights.

Name of the Participant (please print)

Signature of the Participant

Date

Signature of Investigator

Date 
Appendix VIII: Debriefing Form (Experiment 3)

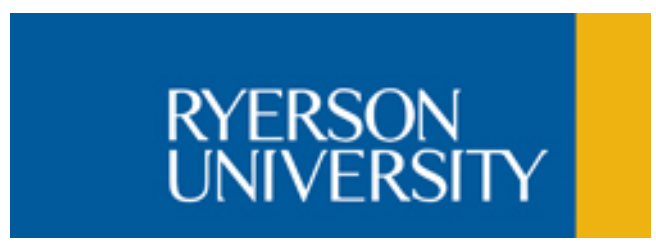

\title{
DEPARTMENT OF PSYCHOLOGY FACULTY OF ARTS
}

\author{
Debriefing \\ Aging, Cognitive Control, and Emotion-II
}

Thank you for your participation!

In this study, we examined how aging and emotion affect cognitive control. Cognitive control is your ability to keep in mind the goals of a task and to adjust behaviour to successfully accomplish these goals. Prior research has suggested that this ability declines as you age. However, your ability to regulate and process emotion or emotional information remains stable, or even improves, with age. This study aimed to examine how emotional functioning influences cognitive control in older adults. Participants completed a cognitive control task that used faces displaying different expressions (e.g., happy, sad, or neutral). It is hypothesized that the performance of older adults is expected to benefit from faces that showed positive emotions. Such results would be consistent with previous research on the topic.

Your participation in this study contributes to the growing research on interactions between aging, cognitive control, and emotion. It will help us better understand the factors that can help support cognitive control in healthy aging adults.

Please do not hesitate to ask the experimenter questions or to raise any concerns you have. Feel free to contact the principal investigators, Linda Truong at 416-979-5000 ext. 4987 or Dr. Lixia Yang at 416-979-5000 ext. 6522 if you have any questions regarding this project. We greatly appreciate your time and participation! 
Appendix IX: Mood Induction Questionnaire

Mood Induction Questionnaire *administered on the computer via E-Prime

\section{Positive \& Negative Induction Conditions:}

Now you will be asked questions about the slideshow you watched earlier in this task, where you heard music and viewed pictures.

Question 1 (familiarity with the induction music):

Prior to this research study, how familiar were you with the music piece you heard in this task?

1. I have never heard this piece before.

2. I might have heard this piece a few times before.

3. I am somewhat familiar with this piece.

4. I am very familiar with this piece.

Question 2 (familiarity with classical music):

How familiar are you with classical music?

1. I have never heard classical music before.

2. I might have heard classical music occasionally before.

3. I am somewhat familiar with classical music.

4. I am very familiar with classical music.

Question 3 (induction processing):

When I was viewing the slideshow, I was actively trying to:

1. Pay attention to the entire slideshow.

2. Pay attention to the music only.

3. Pay attention to the pictures only.

4. Memorize the entire slideshow.

5. Memorize the music only.

6. Memorize the pictures only.

7. None of the above.

Question 4 (induction effectiveness):

On a scale of 1-9, honestly rate whether the slideshow was effective at putting you in a (positive/negative) mood state.

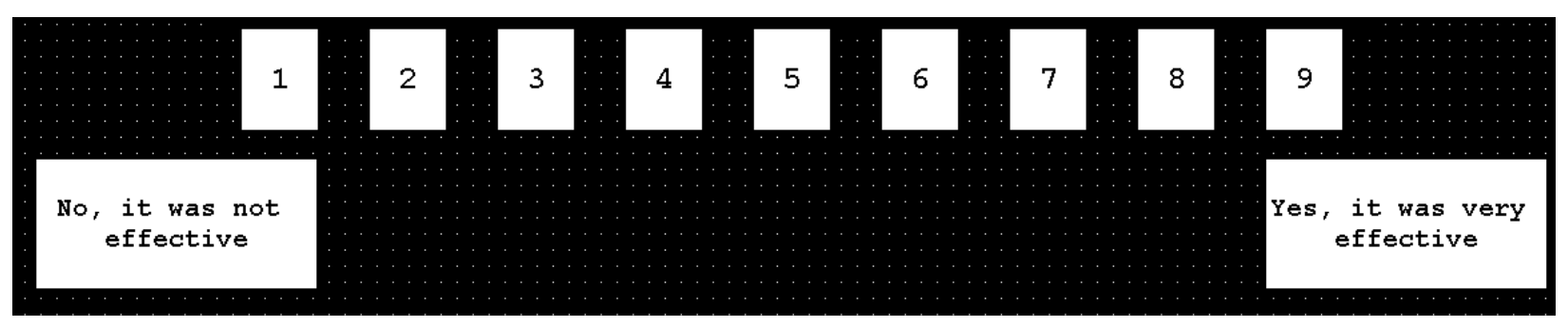




\section{Neutral Mood Induction Condition:}

Now you will be asked questions about the slideshow you watched earlier in this task, where you heard sounds and viewed pictures.

Question 1 (familiarity with the induction sounds):

Prior to this research study, how familiar were you with the sounds you heard in this task?

1. I have never heard these sounds before.

2. I might have heard these sounds a few times before.

3. I am somewhat familiar with these sounds.

4. I am very familiar with these sounds.

Question 2 (induction processing):

When I was viewing the slideshow, I was actively trying to:

1. Pay attention to the entire slideshow.

2. Pay attention to the sounds only.

3. Pay attention to the pictures only.

4. Memorize the entire slideshow.

5. Memorize the sounds only.

6. Memorize the pictures only.

7. None of the above.

Question 3 (induction effectiveness):

On a scale of 1-9, honestly rate whether the slideshow was effective at putting you in a neutral mood state.

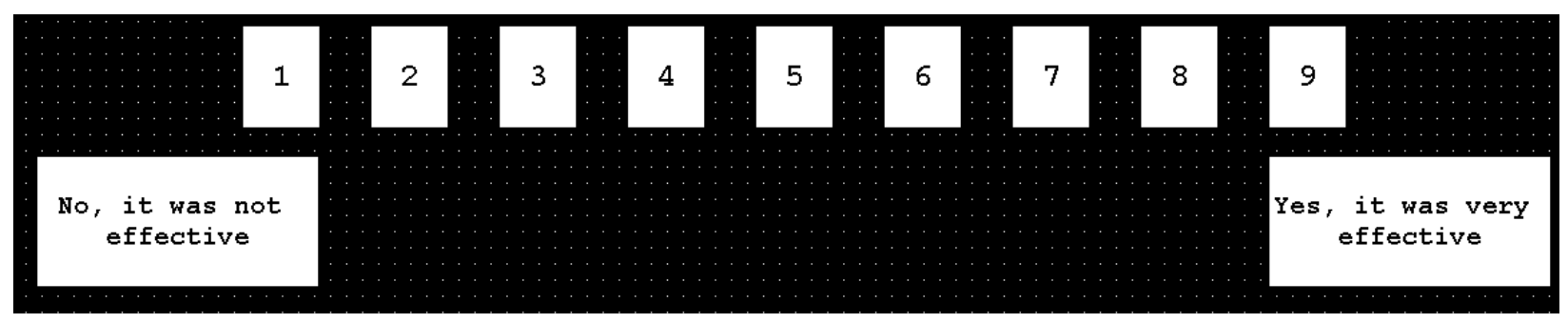


Appendix X: Correlations between AX-CPT Performance and Cognitive Tests

Correlations between AX-CPT Performance and Cognitive Tests (Experiments 1 and 2)

\begin{tabular}{|c|c|c|c|}
\hline $\begin{array}{l}\text { AX-CPT } \\
\text { Performance } \\
\text { Measure } \\
\end{array}$ & $\begin{array}{l}\text { Younger and Older } \\
\text { Adults } \\
(n=266)\end{array}$ & $\begin{array}{l}\text { Younger Adults } \\
(n=134)\end{array}$ & $\begin{array}{l}\text { Older Adults } \\
(n=132)\end{array}$ \\
\hline Probe-lure errors & $\begin{array}{l}\text { Corsi: } r=-.20^{* *} \\
\text { DCCST: } r=-.17^{* *} \\
\text { Vocab: } r=-.13^{*}\end{array}$ & $\begin{array}{l}\text { Corsi: } r=-.21^{* *} \\
\text { DCCST: } r=-.26^{* *} \\
\text { Vocab: } r=-.24^{* *}\end{array}$ & $\begin{array}{l}\text { Corsi: } r=-.36^{*} \\
\text { DCCST: } r=-.20^{*}\end{array}$ \\
\hline Cue-lure errors & $\begin{array}{l}\text { Corsi: } r=.12^{*} \\
\text { Pattern: } r=.15^{*} \\
\text { Vocab: } r=-.16^{* *}\end{array}$ & & DCCST: $r=-.19 *$ \\
\hline $\begin{array}{l}\text { Proactive index- } \\
\text { errors }\end{array}$ & $\begin{array}{l}\text { Corsi: } r=.24 * \\
\text { Pattern: } r=.20^{* *} \\
\text { DCCST: } r=.28^{* *} \\
\text { Flanker: } r=.19^{* *}\end{array}$ & DCCST: $r=.27 * *$ & Corsi: $r=.20^{*}$ \\
\hline $\begin{array}{l}\text { Probe-lure } z \text { - } \\
\text { transformed median } \\
\text { RTs }\end{array}$ & $\begin{array}{l}\text { DCCST: } r=-.23^{*} \\
\text { Flanker: } r=-.13^{*} \\
\text { Vocab: } r=.13^{*}\end{array}$ & & \\
\hline $\begin{array}{l}\text { Cue-lure } z \text { - } \\
\text { transformed median } \\
\text { RTs }\end{array}$ & & DCCST: $r=.18^{*}$ & \\
\hline Proactive index-RTs & DCCST: $r=.15^{*}$ & & \\
\hline \multicolumn{4}{|c|}{ Note. $r$ denotes Pearson coefficients; only significant correlations are presented; Corsi $=$ Corsi Block; Pattern = } \\
\hline
\end{tabular}


Appendix XI: AX-CPT Median Response Times

AX-CPT Median Response Times

\begin{tabular}{|c|c|c|c|c|}
\hline \multirow[t]{2}{*}{ Trial Type } & \multicolumn{2}{|c|}{ Younger Adults } & \multicolumn{2}{|c|}{ Older Adults } \\
\hline & $M$ & $(S D)$ & $M$ & (SD) \\
\hline \multicolumn{5}{|l|}{ Experiment 1A } \\
\hline Control & 432.85 & $(64.72)$ & 542.13 & (99.86) \\
\hline Probe-lure & 482.64 & (124.04) & 693.86 & (228.40) \\
\hline Cue-lure & 523.31 & (59.03) & 600.64 & (72.69) \\
\hline Target & 415.42 & $(60.91)$ & 483.70 & (72.01) \\
\hline \multicolumn{5}{|l|}{ Experiment 1B } \\
\hline Control & 424.04 & (66.94) & 508.09 & (121.06) \\
\hline Probe-lure & 473.23 & (169.39) & 616.67 & (225.22) \\
\hline Cue-lure & 517.93 & (49.38) & 569.91 & (83.10) \\
\hline Target & 409.88 & (77.28) & 470.76 & (122.44) \\
\hline \multicolumn{5}{|l|}{ Experiment 2A } \\
\hline Control & 450.09 & (136.41) & 591.18 & (161.51) \\
\hline Probe-lure & 450.73 & (195.46) & 563.29 & (170.53) \\
\hline Cue-lure & 640.56 & (137.08) & 804.98 & (195.77) \\
\hline Target & 502.09 & (119.70) & 618.60 & (121.64) \\
\hline \multicolumn{5}{|c|}{$\begin{array}{l}\text { Experiment 2B } \\
\text { (negative induction) }\end{array}$} \\
\hline Control & 432.31 & (121.50) & 535.15 & (149.64) \\
\hline Probe-lure & 389.06 & (94.58) & 511.69 & (175.73) \\
\hline Cue-lure & 637.40 & (134.41) & 834.69 & (262.75) \\
\hline Target & 490.19 & (102.52) & 581.59 & (114.07) \\
\hline \multicolumn{5}{|c|}{$\begin{array}{l}\text { Experiment 2B } \\
\text { (positive induction) }\end{array}$} \\
\hline Control & 450.44 & (184.51) & 547.40 & (167.65) \\
\hline Probe-lure & 407.06 & $(140.43)$ & 534.32 & (205.98) \\
\hline Cue-lure & 653.00 & (177.43) & 752.58 & (157.15) \\
\hline Target & 505.93 & (160.53) & 608.50 & (136.69) \\
\hline
\end{tabular}


Appendix XII: Face AX-CPT Questionnaire

\section{Face AX-CPT Task Questions}

** Administer the following questions by asking the participants and then recording a summary of their responses below:

I will now ask you some questions about the task you just finished. You were asked to complete the same task 3 times, in 3 separate blocks:

1. Did you notice any differences in what you saw across the 3 blocks? (you can provide some prompts, such as "any differences in the faces you saw?"). If so, please clarify:

2. Was your performance across the 3 blocks influenced by these differences? (Or: "did your performance change across the 3 blocks?”). Please clarify:

3. Did you use any strategies to help you with your performance on this task? Please clarify: 


\section{References}

Adrover-Roig, D., Sesé, A., Barceló, F., \& Palmer, A. (2012). A latent variable approach to executive control in healthy ageing. Brain and Cognition, 78(3), 284-299. doi:10.1016/j.bandc.2012.01.005

Ashby, F. G., Isen, A. M., \& Turken, U. (1999). A neuropsychological theory of positive affect and its influence on cognition. Psychological Review, 106(3), 529-550. doi:10.1037/0033295X.106.3.529

Barbey, A. K., Koenigs, M., \& Grafman, J. (2013). Dorsolateral prefrontal contributions to human working memory. Cortex, 49(5), 1195-1205. doi:10.1016/j.cortex.2012.05.022

Biss, R. K., \& Hasher, L. (2011). Delighted and distracted: Positive affect increases priming for irrelevant information. Emotion, 11(6), 1474-1478. doi:10.1037/a0023855

Biss, R. K., Weeks, J. C., \& Hasher, L. (2012). Happily distracted: Mood and a benefit of attention dysregulation in older adults. Frontiers in Psychology, 3, 399. doi:10.3389/fpsyg.2012.00399

Bopp, K. L., \& Verhaeghen, P. (2005). Aging and verbal memory span: A meta-analysis. The Journals of Gerontology. Series B, Psychological Sciences and Social Sciences, 60(5), 223233. doi:10.1093/geronb/60.5.P223

Borg, C., Leroy, N., Favre, E., Laurent, B., \& Thomas-Antérion, C. (2011). How emotional pictures influence visuospatial binding in short-term memory in ageing and Alzheimer’s disease? Brain and Cognition, 76, 20-25. doi:10.1016/j.bandc.2011.03.008 
Bradley, M. M., \& Lang, P. J. (1994). Measuring Emotion: The self-assessment manikin and the semantic differential. Journal of Behavior Therapy and Experimental Psychiatry, 25, 4959. doi:10.1016/0005-7916(94)90063-9

Braver, T. S. (2012). The variable nature of cognitive control: a dual mechanisms framework. Trends in Cognitive Sciences, 16(2), 106-113. doi:10.1016/j.tics.2011.12.010

Braver, T. S., \& Barch, D. M. (2002). A theory of cognitive control, aging cognition, and neuromodulation. Neuroscience and Biobehavioral Reviews, 26(7), 809-817. doi:10.1016/S0149-7634(02)00067-2

Braver, T. S., Barch, D. M., Keys, B. A., Carter, C. S., Cohen, J. D., \& Kaye. (2001). Context processing in older adults: Evidence for a theory relating cognitive control to neurobiology in healthy aging. Journal of Experimental Psychology: General, 130(4), 746-763. doi: 10.1037/0096-3445.130.4.746

Braver, T. S., Gray, J. R., \& Burgess, G. C. (2007). Explaining the many varieties of working memory variation: Dual mechanisms of cognitive control. In A. Conway, C. Jarrold, M. Kane, A. Miyake, \& J. Towse (Eds.), Variation in Working Memory (pp. 76-106). New York, NY: Oxford University Press. doi:10.1093/acprof:oso/9780195168648.001.0001

Braver, T. S., Krug, M. K., Chiew, K. S., Kool, W., Westbrook, J. A., Clement, N. J., ... Somerville, L. H. (2014). Mechanisms of motivation-cognition interaction: Challenges and opportunities. Cognitive, Affective \& Behavioral Neuroscience, 14(2), 443-72. doi:10.3758/s13415-014-0300-0 
Braver, T. S., Paxton, J. L., Locke, H. S., \& Barch, D. M. (2009). Flexible neural mechanisms of cognitive control within human prefrontal cortex. Proceedings of the National Academy of Sciences of the United States of America, 106(18), 7351-7356. doi:10.1073/pnas.0808187106

Braver, T. S., Satpute, A. B., Rush, B. K., Racine, C. A., \& Barch, D. M. (2005). Context processing and context maintenance in healthy aging and early stage dementia of the Alzheimer’s type. Psychology and Aging, 20(1), 33-46. doi:10.1037/0882-7974.20.1.33

Braver, T. S., \& West, R. (2008). Working memory, executive control, and aging. In F. I. M. Craik \& T. A. Salthouse (Eds.), The Handbook of Aging and Cognition (3rd ed., pp. 311372). New York, NY: Psychology Press.

Bugg, J. M. (2014). Evidence for the sparing of reactive cognitive control with age. Psychology and Aging, 29, 115-127. doi:10.1037/a0035270

Carpenter, S. M., Peters, E., Västfjäll, D., \& Isen, A. M. (2013). Positive feelings facilitate working memory and complex decision making among older adults. Cognition \& Emotion, 27(1), 184-192. doi:10.1080/02699931.2012.698251

Carstensen, L. L., Isaacowitz, D. M., \& Charles, S. T. (1999). Taking time seriously: A theory of socioemotional selectivity. American Psychologist, 54(3), 165-181. doi: 10.1037//0003066X.54.3.165

Carstensen, L. L., \& Lang, F. R. (1995). Future Time Perspective Scale. Stanford, CA: Stanford University. 
Carstensen, L. L., Pasupathi, M., Mayr, U., \& Nesselroade, J. R. (2000). Emotional experience in everyday life across the adult life span. Journal of Personality and Social Psychology, 79(4), 644-655. doi:10.1037/0022-3514.79.4.644

Carstensen, L. L., Turan, B., Scheibe, S., Ram, N., Ersner-Hershfield, H., Samanez-Larkin, G. R., ... Nesselroade, J. R. (2011). Emotional experience improves with age: Evidence based on over 10 years of experience sampling. Psychology and Aging, 26(1), 21-33. doi:10.1037/a0021285

Charles, S. T., \& Carstensen, L. L. (2010). Social and emotional aging. Annual Review of Psychology, 61, 383-409. doi:10.1146/annurev.psych.093008.100448

Charles, S. T., Mather, M., \& Carstensen, L. L. (2003). Aging and emotional memory: The forgettable nature of negative images for older adults. Journal of Experimental Psychology: General, 132(2), 310-324. doi:10.1037/0096-3445.132.2.310

Chiew, K. S., \& Braver, T. S. (2011). Positive affect versus reward: Emotional and motivational influences on cognitive control. Frontiers in Psychology, 2, 279. doi:10.3389/fpsyg.2011.00279

Chiew, K. S., \& Braver, T. S. (2014). Dissociable influences of reward motivation and positive emotion on cognitive control. Cognitive, Affective \& Behavioral Neuroscience, 14(2), 509529. doi:10.3758/s13415-014-0280-0 
Chu, O. (2014). The effect of mood on set-switching abilities in younger and older adults. (Doctoral dissertation). Retrieved from ProQuest Dissertations and Theses database. (NR98636).

Cohen, J. D., Barch, D. M., Carter, C., \& Servan-Schreiber, D. (1999). Context-processing deficits in schizophrenia: Converging evidence from three theoretically motivated cognitive tasks. Journal of Abnormal Psychology, 108(1), 120-133. doi:10.1037/0021843X.108.1.120

Czernochowski, D., Nessler, D., \& Friedman, D. (2010). On why not to rush older adults-relying on reactive cognitive control can effectively reduce errors at the expense of slowed responses. Psychophysiology, 47(4), 637-646. doi:10.1111/j.1469-8986.2009.00973.x

Diamond, A. (2013). Executive functions. Annual Review of Psychology, 64, 135-168. doi:10.1146/annurev-psych-113011-143750

Dolcos, F., Iordan, A., \& Dolcos, S. (2011). Neural correlates of emotion-cognition interactions: A review of evidence from brain imaging investigations. Journal of Cognitive Psychology, 23(6), 669-694. doi: 10.1080/20445911.2011.594433

Dreisbach, G. (2006). How positive affect modulates cognitive control: The costs and benefits of reduced maintenance capability. Brain and Cognition, 60, 11-19. doi:10.1016/j.bandc.2005.08.003 
Dreisbach, G., \& Goschke, T. (2004). How positive affect modulates cognitive control: Reduced perseveration at the cost of increased distractibility. Journal of Experimental Psychology. Learning, Memory, and Cognition, 30(2), 343-353. doi:10.1037/0278-7393.30.2.343

Dyck, M., Loughead, J., Kellermann, T., Boers, F., Gur, R. C., \& Mathiak, K. (2011). Cognitive versus automatic mechanisms of mood induction differentially activate left and right amygdala. NeuroImage, 54(3), 2503-2513. doi:10.1016/j.neuroimage.2010.10.013

Ebner, N. C., Riediger, M., \& Lindenberger, U. (2010). FACES-A database of facial expressions in young, middle-aged, and older women and men: Development and validation. Behavior Research Methods, 42(1), 351-362. doi:10.3758/BRM.42.1.351

Erdfelder, E., Faul, F. \& Buchner, A. (1996). GPOWER: A general power analysis program. Behavior Research Methods, Instruments, \& Computers, 28, 1-11.

Fan, J., McCandliss, B. D., Sommer, T., Raz, A., \& Posner, M. I. (2002). Testing the efficiency and independence of attentional networks. Journal of Cognitive Neuroscience, 14(3), 340347. doi:10.1162/089892902317361886

Fröber, K., \& Dreisbach, G. (2012). How positive affect modulates proactive control: Reduced usage of informative cues under positive affect with low arousal. Frontiers in Psychology, 3, 1-14. doi:10.3389/fpsyg.2012.00265

Fröber, K., \& Dreisbach, G. (2014). The differential influences of positive affect, random reward, and performance-contingent reward on cognitive control. Cognitive, Affective \& Behavioral Neuroscience, 14(2), 530-547. doi:10.3758/s13415-014-0259-х 
Gazzaley, A., Cooney, J. W., Rissman, J., \& D’Esposito, M. (2005). Top-down suppression deficit underlies working memory impairment in normal aging. Nature Neuroscience, 8(10), 1298-1300. doi:10.1038/nn1543

Haarmann, H. J., Ashling, G. E., Davelaar, E. J., \& Usher, M. (2005). Age-related declines in context maintenance and semantic short-term memory. The Quarterly Journal of Experimental Psychology., 58, 34-53. doi:10.1080/02724980443000214

Hasher, L., \& Zacks, R. T. (1988). Working memory, comprehension, and aging: A review and a new view. In G. H. Bower (Ed.), The Psychology of Learning and Motivation: Advances in research and theory (Volume 22, pp. 193-225). New York, NY: Academic Press.

Hedden, T., \& Gabrieli, J. D. E. (2004). Insights into the ageing mind: A view from cognitive neuroscience. Nature Reviews: Neuroscience, 5(2), 87-96. doi:10.1038/nrn1323

Hess, T. M. (2005). Memory and aging in context. Psychological Bulletin, 131(3), 383-406. doi:10.1037/0033-2909.131.3.383

Isaacowitz, D. M. (2012). Mood regulation in real-time: Age differences in the role of looking. Current Directions in Psychological Science, 21(4), 237-242. doi:10.1177/0963721412448651

Isaacowitz, D. M., \& Blanchard-Fields, F. (2012). Linking process and outcome in the study of emotion and aging. Perspectives on Psychological Science, 7(1), 3-17. doi:10.1177/1745691611424750 
Isaacowitz, D. M., \& Noh, S. R. (2011). Does looking at the positive mean feeling good? Age and individual differences matter. Social and Personality Psychology Compass, 5(8), 505517. doi:10.1111/j.1751-9004.2011.00374.x

Isaacowitz, D. M., Toner, K., Goren, D., \& Wilson, H. R. (2008). Looking while unhappy: Mood-congruent gaze in young adults, positive gaze in older adults. Psychological Science, 19(9), 848-853. doi:10.1111/j.1467-9280.2008.02167.x

Isaacowitz, D. M., Toner, K., \& Neupert, S. D. (2009). Use of gaze for real-time mood regulation: Effects of age and attentional functioning. Psychology and Aging, 24(4), 989994. doi:10.1037/a0017706

Jimura, K., \& Braver, T. S. (2010). Age-related shifts in brain activity dynamics during task switching. Cerebral Cortex, 20(6), 1420-1431. doi:10.1093/cercor/bhp206

Jurado, M. B., \& Rosselli, M. (2007). The elusive nature of executive functions: A review of our current understanding. Neuropsychology Review, 17(3), 213-233. doi:10.1007/s11065-007$9040-\mathrm{z}$

Kane, M. J., \& Engle, R. W. (2002). The role of prefrontal cortex in working-memory capacity, executive attention, and general fluid intelligence: An individual-differences perspective. Psychonomic Bulletin \& Review, 9(4), 637-671. doi: 10.3758/BF03196323

Katzman, R., Brown, T., Fuld, P., Peck, A., Schechter, R., \& Schimmel, H. (1983). Validation of a short Orientation-Memory-Concentration Test of cognitive impairment. The American Journal of Psychiatry, 140, 734-739. 
Kellough, J. L., \& Knight, B. G. (2011). Positivity effects in older adults ' perception of facial emotion : The role of future time perspective. The Journals of Gerontology Series B: Psychological Sciences and Social Sciences, 67(2), 150-158. doi:10.1093/geronb/gbr079.

Knight, M., Seymour, T. L., Gaunt, J. T., Baker, C., Nesmith, K., \& Mather, M. (2007). Aging and goal-directed emotional attention: Distraction reverses emotional biases. Emotion, 7(4), 705-714. doi:10.1037/1528-3542.7.4.705

Kray, J., Schmitt, H., \& Heintz, S. (2015). Does verbal labeling influence age differences in proactive and reactive cognitive control? Developmental Psychology, 51(3), 378-391. doi: 10.1037/a0038795

Lang, P. J., Bradley, M. M., \& Cuthbert, B. N. (2008). International affective picture system (IAPS): Affective ratings of pictures and instruction manual. Technical Report A-8. University of Florida, Gainesville, Fl.

Larcom, M. J., \& Isaacowitz, D. M. (2009). Rapid emotion regulation after mood induction: Age and individual differences. Journals of Gerontology: Series B Psychological Sciences and Social Sciences, 64(6), 733-741. doi:10.1093/geronb/gbp077

Locke, H. S., \& Braver, T. S. (2008). Motivational influences on cognitive control: Behavior, brain activation, and individual differences. Cognitive, Affective, \& Behavioral Neuroscience, 8(1), 99-112. doi:10.3758/CABN.8.1.99 
Löckenhoff, C. E., \& Carstensen, L. L. (2007). Aging, emotion, and health-related decision strategies: Motivational manipulations can reduce age differences. Psychology and Aging, 22(1), 134-146. doi:10.1037/0882-7974.22.1.134

Loria, T., \& Russo, F. A. (2012). Do urban soundscapes influence visual attention? Proceedings of Acoustics Week in Canada, Canadian Acoustics, 40, 126-128.

Lorsbach, T. C., \& Reimer, J. F. (2008). Context processing and cognitive control in children and young adults. The Journal of Genetic Psychology, 169, 34-50. doi:10.3200/GNTP.169.1.34-50

Lorsbach, T. C., \& Reimer, J. F. (2010). Developmental differences in cognitive control: Goal representation and maintenance during a continuous performance task. Journal of Cognition and Development, 11(2), 185-216. doi:10.1080/15248371003699936

Lovibond, P. F., \& Lovibond, S. H. (1995). Manual for the Depression Anxiety Stress Scales. Sydney: The Psychology Foundation of Australia.

Manard, M., Carabin, D., Jaspar, M., \& Collette, F. (2014). Age-related decline in cognitive control: The role of fluid intelligence and processing speed. BMC Neuroscience, 15:7, 1-16. doi:10.1186/1471-2202-15-7

Mather, M., \& Carstensen, L. L. (2003). Aging and attentional biases for emotional faces. Psychological Science, 14(5), 409-415. doi:10.1111/1467-9280.01455 
Mather, M., \& Carstensen, L. L. (2005). Aging and motivated cognition: The positivity effect in attention and memory. Trends in Cognitive Sciences, 9(10), 496-502. doi:10.1016/j.tics.2005.08.005

Mather, M., \& Knight, M. (2005). Goal-directed memory: The role of cognitive control in older adults’ emotional memory. Psychology and Aging, 20(4), 554-570. doi:10.1037/08827974.20.4.554

Mikels, J. A., Larkin, G. R., Reuter-Lorenz, P. A., \& Cartensen, L. L. (2005). Divergent trajectories in the aging mind: Changes in working memory for affective versus visual information with age. Psychology and Aging, 20(4), 542-553. doi:10.1037/08827974.20.4.542

Mitchell, R. L. C., \& Phillips, L. H. (2007). The psychological, neurochemical and functional neuroanatomical mediators of the effects of positive and negative mood on executive functions. Neuropsychologia, 45(4), 617-629. doi:10.1016/j.neuropsychologia.2006.06.030

Murphy, N. A., \& Isaacowitz, D. M. (2008). Preferences for emotional information in older and younger adults: A meta-analysis of memory and attention tasks. Psychology and Aging, 23(2), 263-286. doi:10.1037/0882-7974.23.2.263

Noh, S. R., Larcom, M. J., Liu, X., \& Isaacowitz, D. M. (2012). The role of affect in attentional functioning for younger and older adults. Frontiers in Psychology, 3, 311. doi:10.3389/fpsyg.2012.00311 
Northwestern University and the National Institutes of Health. [NIH Toolbox]. (2012). Retrieved from http://www.nihtoolbox.org

Oaksford, M., Morris, F., Grainger, B., \& Williams, J. M. G. (1996). Mood, reasoning , and central executive processes. Journal of Experimental Psychology: Learning, Memory, and Cognition, 22, 476-492. doi:10.1037/0278-7393.22.2.476

Paxton, J. L., Barch, D. M., Racine, C. A., \& Braver, T. S. (2008). Cognitive control, goal maintenance, and prefrontal function in healthy aging. Cerebral Cortex, 18(5), 1010-1028. doi:10.1093/cercor/bhm135

Paxton, J. L., Barch, D. M., Storandt, M., \& Braver, T. S. (2006). Effects of environmental support and strategy training on older adults’ use of context. Psychology and Aging, 21(3), 499-509. doi:10.1037/0882-7974.21.3.499

Phillips, L. H., Slessor, G., Bailey, P. E., \& Henry, J. D. (2014). Older adults’ perceptions of social and emotional cues. In P. Verhaeghen \& C. K. Hertzog (Eds.), The Oxford Handbook of Emotion, Social Cognition, and Problem Solving in Adulthood (pp. 9-25). New York, NY: Oxford University Press.

Phillips, L. H., Smith, L., \& Gilhooly, K. J. (2002). The effects of adult aging and induced positive and negative mood on planning. Emotion, 2(3), 263-272. doi:10.1037/15283542.2.3.263

Psychology Software Tools, Inc. [E-Prime 2.0]. (2012). Retrieved from http://www.pstnet.com. 
Redick, T. S. (2014). Cognitive control in context: Working memory capacity and proactive control. Acta Psychologica, 145, 1-9. doi:10.1016/j.actpsy.2013.10.010

Reed, A. E., \& Carstensen, L. L. (2012). The theory behind the age-related positivity effect. Frontiers in Psychology, 3, 339. doi:10.3389/fpsyg.2012.00339

Reed, A. E., Chan, L., \& Mikels, J. a. (2014). Meta-analysis of the age-related positivity effect: Age differences in preferences for positive over negative information. Psychology and Aging, 29, 1-15. doi:10.1037/a0035194

Reimer, J. F., Radvansky, G. A., Lorsbach, T. C., \& Armendarez, J. J. (2015). Event structure and cognitive control. Journal of Experimental Psychology:Learning, Memory, and Cognition. Advance online publication. doi: 10.1037/xlm0000105

Richmond, L. L., Redick, T. S., Braver, T. S., Richmond, L. L., \& Redick, T. S. (2015). Remembering to prepare: The benefits (and costs) of high working memory capacity. Journal of Experimental Psychology: Learning, Memory, and Cognition, (Advance online publication). doi:10.1037/xlm0000122

Rosvold, H. E., Mirsky, A. F., Sarason, I., Bransome, E. D., \& Beck, L. H. (1956). A continuous performance test of brain damage. Journal of Consulting Psychology, 20(5), 343-350. doi: $10.1037 / \mathrm{h} 0043220$

Rowe, G., Hasher, L., \& Turcotte, J. (2009). Age and synchrony effects in visuospatial working memory. Quarterly Journal of Experimental Psychology, 62(10), 1873-1880. doi:10.1080/17470210902834852 
Rush, B. K., Barch, D. M., \& Braver, T. S. (2006). Accounting for cognitive aging: context processing, inhibition or processing speed? Neuropsychology, Development, and Cognition: Section B, Aging, Neuropsychology and Cognition, 13(3-4), 588-610. doi:10.1080/13825580600680703

Russo, F. A., Vempala, N. N., \& Sandstrom, G. M. (2013). Predicting musically induced emotions from physiological inputs: Linear and neural network models. Frontiers in Psychology, 4(468), 1-8. doi:10.3389/fpsyg.2013.00468

Salthouse, T. A., Atkinson, T. M., \& Berish, D. E. (2003). Executive functioning as a potential mediator of age-related cognitive decline in normal adults. Journal of Experimental Psychology. General, 132(4), 566-594. doi:10.1037/0096-3445.132.4.566

Samanez-Larkin, G. R., Robertson, E. R., Mikels, J. A., Carstensen, L. L., \& Gotlib, I. H. (2009). Selective attention to emotion in the aging brain. Psychology and Aging, 24(3), 519-529. doi:10.1037/a0016952

Scheibe, S., \& Blanchard-Fields, F. (2009). Effects of regulating emotions on cognitive performance: What is costly for young adults is not so costly for older adults. Psychology and Aging, 24(1), 217-223. doi:10.1037/a0013807

Scheibe, S., \& Carstensen, L. L. (2010). Emotional aging: Recent findings and future trends. Journal of Gerontology: Psychological Sciences, 65B(2), 135-144. doi:10.1093/geronb/gbp132. 
Schmitt, H., Ferdinand, N. K., \& Kray, J. (2015). The influence of monetary incentives on context processing in younger and older adults: An event-related potential study. Cognitive, Affective, \& Behavioral Neuroscience, 15(2), 416-434. doi:10.3758/s13415-015-0335-x

Shipley, W. C. (1940). A self-administering scale for measuring intellectual impairment and deterioration. Journal of Psychology: Interdisciplinary and Applied, 9, 371-337. doi:10.1080/00223980.1940.9917704

Staub, B., Doignon-Camus, N., Bacon, É., \& Bonnefond, A. (2014). Age-related differences in the recruitment of proactive and reactive control in a situation of sustained attention. Biological Psychology, 103, 38-47. doi:10.1016/j.biopsycho.2014.08.007

Truong, L., \& Yang, L. (2014). Friend or foe? Decoding the facilitative and disruptive effects of emotion on working memory in younger and older adults. Frontiers in Psychology, 5(94), 1-9. doi:10.3389/fpsyg.2014.00094

van Wouwe, N. C., Band, G. P. H., \& Ridderinkhof, K. R. (2009). Proactive control and episodic binding in context processing effects. Acta Psychologica, 131(3), 245-253. doi:10.1016/j.actpsy.2009.05.003

van Wouwe, N. C., Band, G. P. H., \& Ridderinkhof, K. R. (2011). Positive affect modulates flexibility and evaluative control. Journal of Cognitive Neuroscience, 23(3), 524-539. doi:10.1162/jocn.2009.21380 
Volkow, N. D., Gur, R. C., Wang, G. J., Fowler, J. S., Moberg, P. J., Ding, Y. S., ... Logan, J. (1998). Association between decline in brain dopamine activity with age and cognitive and motor impairment in healthy individuals. American Journal of Psychiatry, 155(3), 344-349.

Watson, D., Clark, L. A., \& Tellegen, A. (1988). Development and validation of brief measures of positive and negative affect: The PANAS scales. Journal of Personality and Social Psychology, 54, 1063-1070. doi:10.1037/0022-3514.54.6.1063

Wechsler, D. (1981). Manual for the Wechsler Adult Intelligence Scale-Revised. New York, NY: Psychological Corporation. 